\title{
Some Chemical \\ Characteristics of \\ Mine Drainage in \\ Illinois
}

By L. G. TOLER

GEOLOGICALSURVEY WATER-SUPPLY PAPER 2078

Prepared in cooperation with the

U.S. Environmental Protection Agency

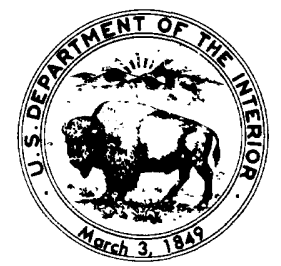

UNITED STATES GOVERNMENT PRINTING OFFICE, WASHINGTON : 1982 
UNITED STATES DEPARTMENT OF THE INTERIOR

JAMES G. WATT, Secretary

GEOLOGICAL SURVEY

Dallas L. Peck, Director

For sale by the Superintendent of Documents, U.S. Government Printing Office Washington, D.C. 20402 


\section{CONTENTS}

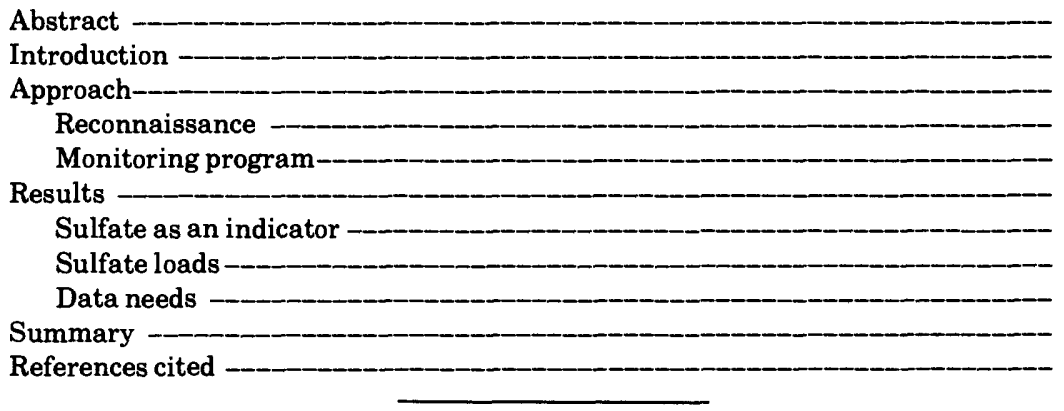

\section{ILLUSTRATIONS}

FigurE 1. Map showing location of Eastern Interior Coal Field

2. Map showing acreage distribution of strip-mined land in Illinois, by county, as of June 1974

3. Map showing location of reconnaissance sampling sites in Illinois _-_ 5

4. Map showing location of and number identifying sampling sites in Big Muddy and Saline River basins, Illinois

5. Graph of field and laboratory alkalinity measurements-_____ 8

6. Generalized flow-duration curve for sites less than $100 \mathrm{mi}^{2}$ in the Big Muddy and Saline River basins, Illinois

7. Generalized flow-duration curve for sites greater than $100 \mathrm{mi}^{2}$ in the Big Muddy and Saline River basins, Illinois

8. Graph showing relationship of annual sulfate load to area of surfacemined land-

9. Graph showing relationship of annual sulfate load per square mile of drainage area to percentage of surface-mined land

10. Graph showing relationship of specific conductance to dissolved solids and sulfate concentrations for Big Muddy River at Murphysboro, Illinois 
TABLE 1. Station data for sulfate concentration and sulfate load computations --

2. Tabulation of average annual sulfate load for site 21 by use of the flowduration curve technique 9

3. Drainage areas, mined areas, and sulfate loads 13

4. Chemical analyses of samples collected at reconnaissance sites 13

5. Chemical analyses of samples from Saline and Big Muddy River basins

\section{FACTORS FOR CONVERTING INCH-POUND UNITS TO INTERNATIONAL SYSTEM (SI) UNITS}

The following factors may be used to convert the inch-pound units published herein to the International System of Units (SI):

\begin{tabular}{lll}
\multicolumn{1}{c}{ Multiply inch-pound unit } & \multicolumn{1}{c}{ By } & \multicolumn{1}{c}{ To obtain SI unit } \\
acre & 0.4047 & square hectometer $\left(\mathrm{hm}^{2}\right)$ \\
cubic foot per second $\left(\mathrm{ft}^{2} / \mathrm{s}\right)$ & 0.02832 & cubic meter per second $\left(\mathrm{m}^{3} / \mathrm{s}\right)$ \\
mile (mi) & 1.609 & kilometer $(\mathrm{km})$ \\
square mile $\left(\mathrm{mi}^{2}\right)$ & 2.590 & square $\mathrm{kilometer}\left(\mathrm{km}^{2}\right)$ \\
ton (short) & 0.9072 & megagram $(\mathrm{Mg})$ \\
tons per day (ton/d) & 0.9072 & megagrams per day $(\mathrm{Mg} / \mathrm{d})$ \\
tons per square mile (ton $\left./ \mathrm{mi}^{2}\right)$ & 0.3503 & megagrams per square kilometer $\left(\mathrm{Mg} / \mathrm{km}^{2}\right)$
\end{tabular}




\title{
SOME CHEMICAL CHARACTERISTICS OF MINE DRAINAGE IN ILLINOIS
}

\author{
By L. G. TOLER
}

ABSTRACT

Surface mining for coal in Illinois has affected runoff from the mined areas and altered water quality in the streams. Average annual sulfate loads in streams are 3,000-4,000 tons per square mile of mined land in the Big Muddy and Saline River basins in southern Illinois. Relatively high concentrations of dissolved aluminum, arsenic, chromium, copper, iron, manganese, and zinc are commonly associated with concentrations of sulfate greater than about 2,000 milligrams per liter.

\section{INTRODUCTION}

Illinois is one of the major coal producing States in the United States. In 1976, Illinois ranked fourth among the States, with a production of approximately 58 million tons. It has the largest reserves of bituminous coal and in 1974 was the State with the second highest total reserves (Smith and Stall, 1975, p. 12).

The coal areas in Illinois are part of the Eastern Interior Coal Field (fig. 1), which includes most of Illinois and parts of Indiana and Kentucky. The coal beds are included in a large structural basin, and in Illinois, their maximum depth is about 1,200 feet near the center of the basin (Smith and Stall, 1975). Near the periphery of the basin, coal is at or near the surface and is amenable to surface mining. About 47 percent of the 1976 production was by surface mining.

As of 1974, approximately 181,000 acres of land in Illinois had been surface mined for coal (Haynes and Klimstra, 1975, p. 2). Figure 2 shows the acreage of surface-mined land in Illinois by county.

Surface mining involves stripping the overburden from the coal, removing the coal, and filing the newly mined pit with overburden from adjacent areas. Surface-mining methods are commonly used to recover coal from depths of up to about 100 feet. Enormous amounts of earth materials are thus disturbed and made more vulnerable to weathering. 


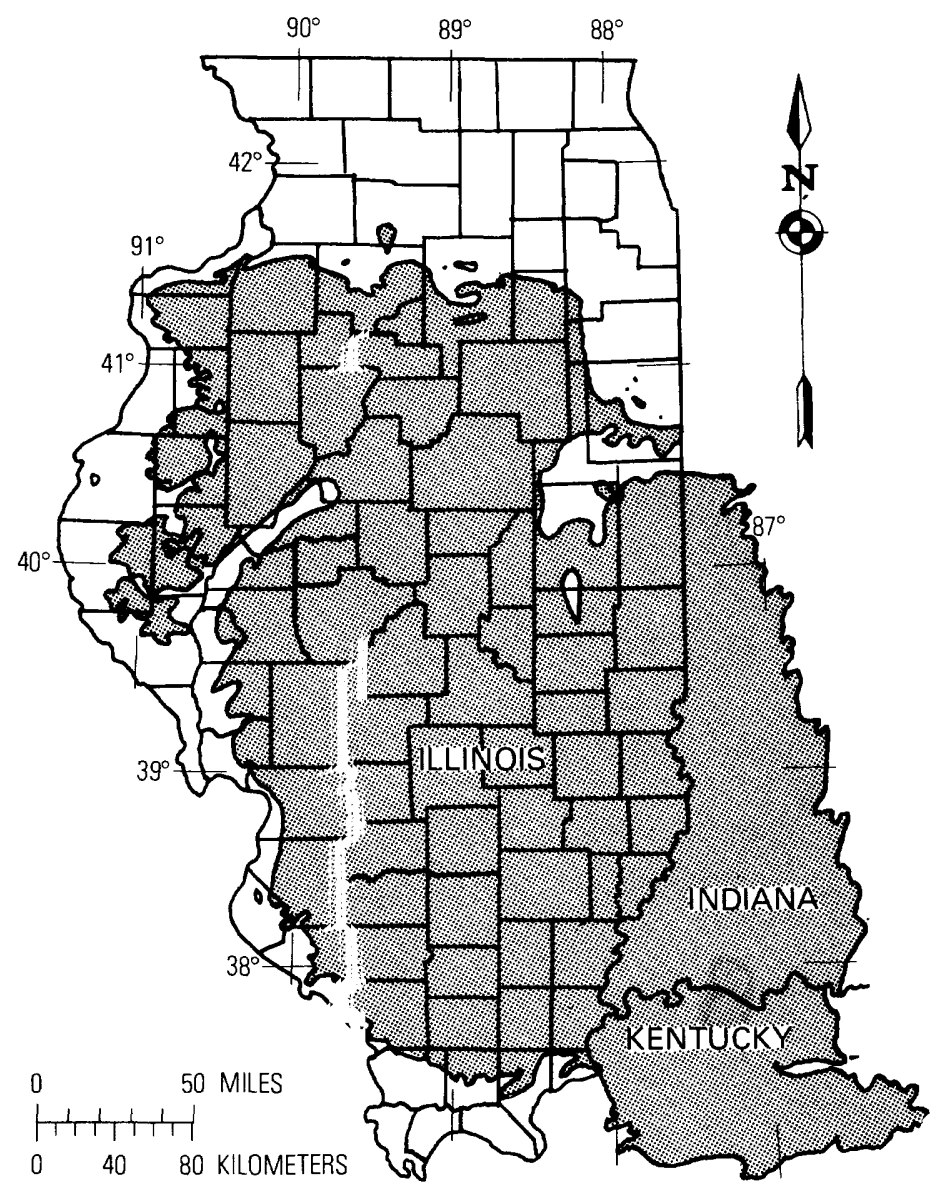

FiguRE 1.-Location of Eastern Interior Coal Field (shaded area).

An apparent increasing demand on our own energy sources has created a high priority for assessments of the effects of surface mining on water quality. In 1974, the U.S. Geological Survey began a program of sampling and evaluating the water quality of streams in the strip-mined areas in Illinois. The U.S. Environmental Protection Agency (EPA) provided funds for the project.

\section{APPROACH}

The first phase of the sampling program was to provide broad geographical coverage of much of the surface-mined area in Illinois. Fifty sites were selected and sampled twice in 1975. 


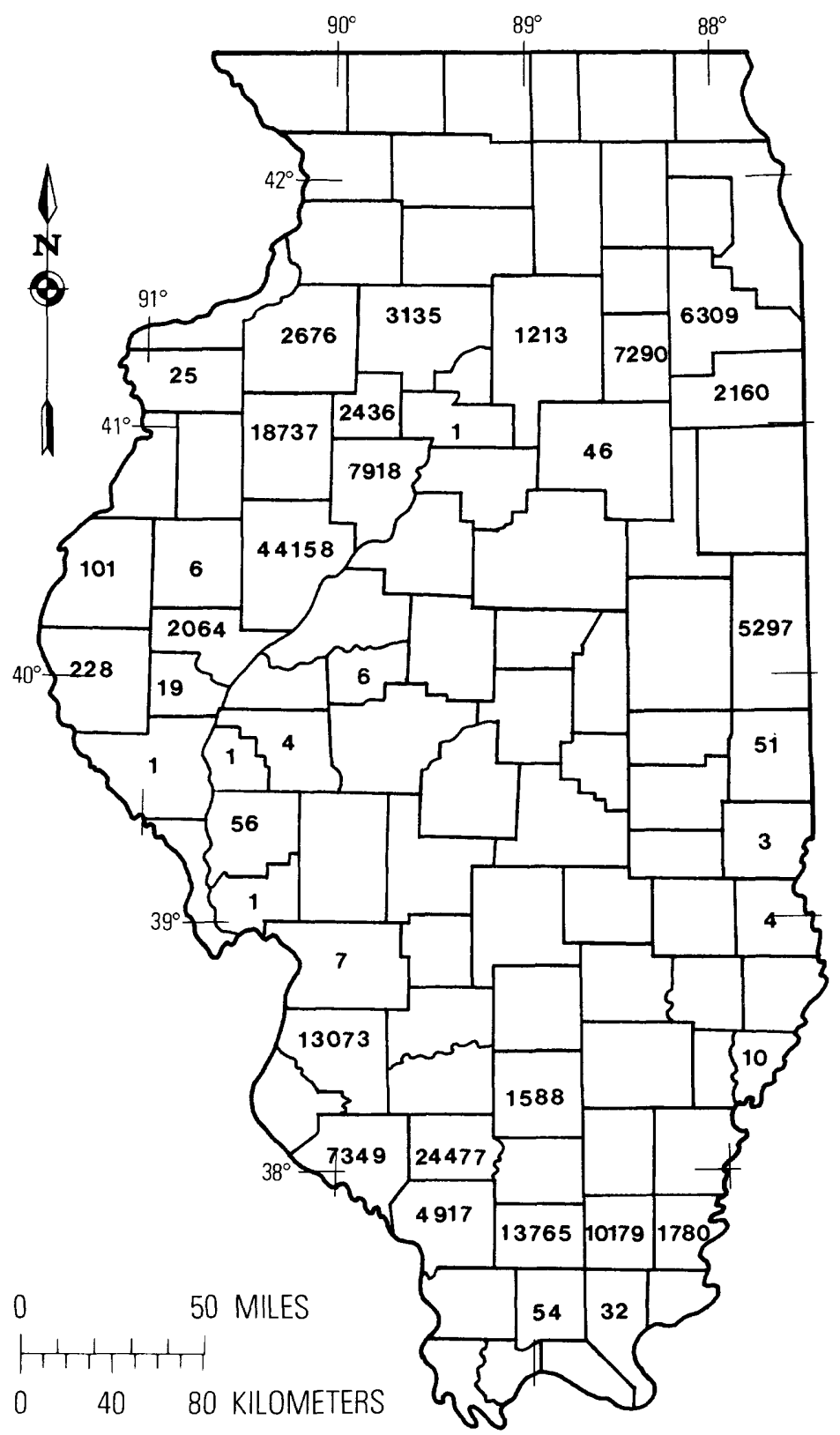

FIGURE 2.-Acreage distribution of strip-mined land in Illinois, by county, as of June 1974 . 
The results of the reconnaissance sampling were then used to design the second phase of the sampling program. This phase included a wider range of hydrologic conditions. The discharge and constituent concentrations obtained during the more intensive sampling were analyzed by regression techniques and extrapolated by use of flow duration curves of discharge to estimate the average quantities of material dissolved in surface-mine drainage. The loads carried by the streams were then related to the mined area within each basin.

\section{RECONNAISSANCE}

The 50 sites selected for the reconnaissance phase are shown in figure 3. These sites were sampled twice, once in May and once in June 1975. All samples were collected during periods of average to low flow, at about the 30 percent flow duration in May and at the 30-65 percent flow duration in June.

Discharge, water temperature, specific conductance, dissolved oxygen, and $\mathrm{pH}$ were measured in the field. Water samples were collected for laboratory analyses of alkalinity, total organic carbon, chloride, phenols, sulfate, sulfide, and the metals aluminum, arsenic, chromium, copper, iron, manganese, mercury, and zinc. The data were then scrutinized for consistency between the two sampling dates and for constituent characteristics common to all or part of the State. Field and laboratory data collected during the reconnaissance sampling are included in table 4.

Sulfate was a major constituent in samples from all sites. Sulfate concentrations ranged from 25 to $4,100 \mathrm{mg} / \mathrm{L}$ (milligrams per liter). On the basis of comparisons with streams having little or no upstream mining activity, it is probable that concentrations of sulfate of more than about $100 \mathrm{mg} / \mathrm{L}$ in base flow are attributable to drainage from mined areas.

The acidic waters contained significant concentrations of aluminum, arsenic, chromium, copper, mercury, and zinc and high concentrations of the more common metals, iron and manganese.

\section{MONITORING PROGRAM}

The heavily mined area that extends west to east across the Big Muddy and Saline River basins in southern Illinois was selected for more intensive sampling because the reconnaissance samples in these basins had the highest concentrations of sulfate and many of the metals and because of concurrent studies in that area by the Greater Egypt Regional Planning and Development Commission (GERPDC) and the Southeastern Illinois Regional Planning and Development Commission (SIRPDC). 


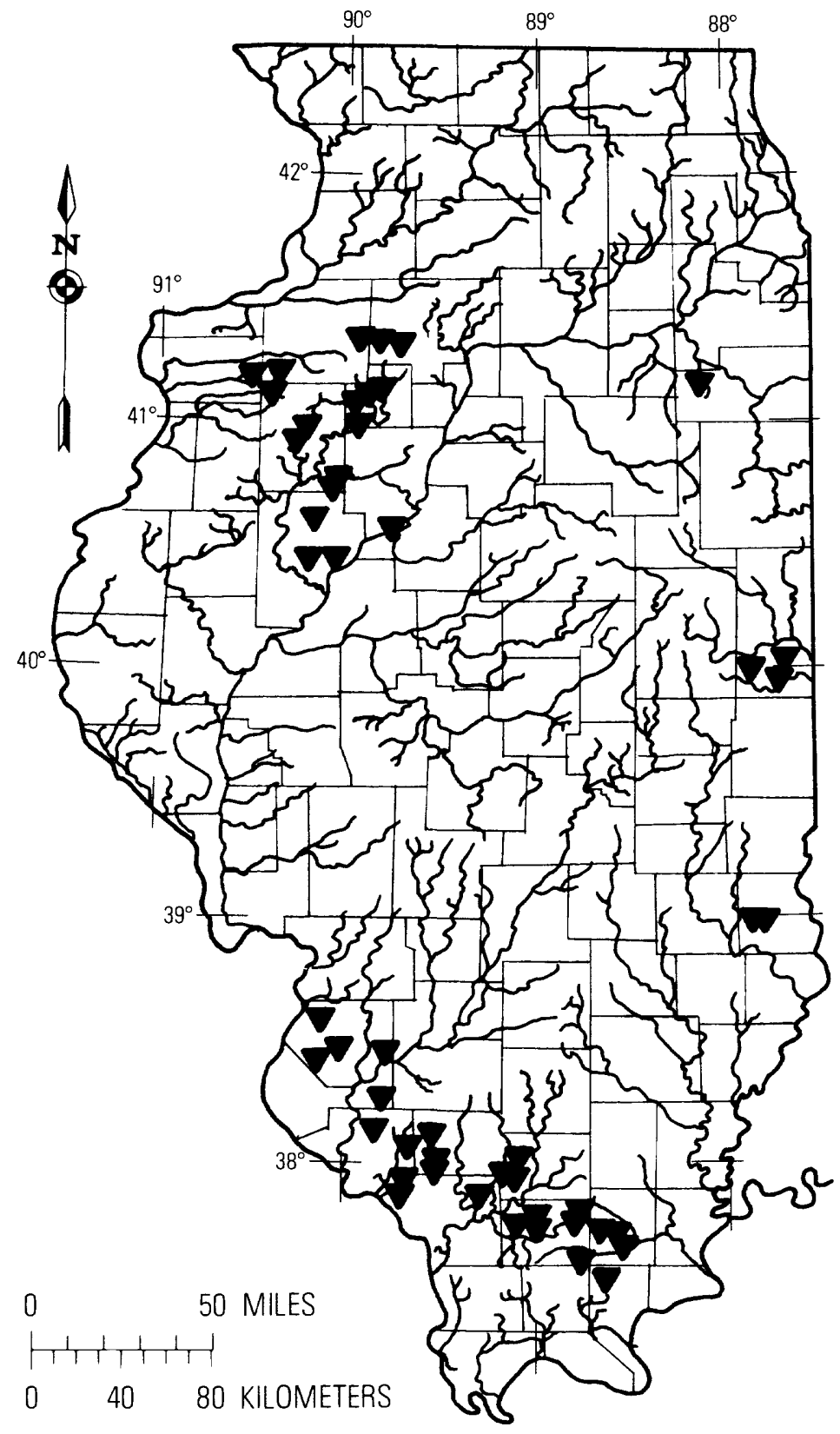

FiguRE 3.-Location of reconnaissance sampling sites in Illinois. 
The selection of 20 sites (fig. 4) was coordinated with the above agencies and the Illinois Environmental Protection Agency (IEPA). Survey personnel collected water-quality samples and made concurrent discharge measurements at these sites about three to four times per year. Where feasible, reference points were located from which water-surface elevations were determined concurrently with discharge measurements and rating curves were prepared from which estimates of discharge could be made. IEPA personnel also collected and analyzed samples and measured water-surface elevation at selected sites about twice per month from March to September 1976. All available analyses are reported in table 5 .

The laboratory analyses of reconnaissance samples did not always show the carbonate-ion and bicarbonate-ion distribution expected from the field $\mathrm{pH}$. Often no carbonate was reported, even though the field $\mathrm{pH}$ was above 8.3 , a condition under which it should occur (Brown, Skougstad, and Fishman, 1970, p. 42). The absence of carbonate indicated that the $\mathrm{pH}$ was changing between the time of sampling and the time of analysis. Because the determination of the carbonate-ion and bicarbonate-ion distribution is a part of the determination of alkalinity, the alkalinity values reported by the laboratory were also suspected of being erroneous. Therefore, alkalinity was determined both in the field and in the laboratory.

The relationship shown in figure 5 dispelled any suspicion of erroneous laboratory alkalinity values even though the $\mathrm{pH}$ changed enroute to the laboratory. However, the exercise indicated that the carbonate-ion and bicarbonate-ion distribution as it existed in the stream must be determined in the field for all samples having the carbonate-ion in solution ( $\mathrm{pH}$ greater than 8.3 ). This was done in addition to the other field analyses for all samples collected by the Survey in the 1976 and 1977 water years.

Drainage areas for all sites, including the reconnaissance sites, were outlined and planimetered on Survey topographic maps. Boundaries of strip-mined areas for the 20 sites sampled during the 1976 and 1977 water years were transferred from GERPDC and SIRPDC (1977) maps to topographic maps, and the area of strip-mined land above the sampling site was planimetered for each of the 20 basins.

\section{RESULTS}

\section{SULFATE AS AN INDICATOR}

The formation of acidic mine water has been documented many times, and an extensive bibliography on the subject is available (Office of Water Resources Research, 1975). Generally, surface mining exposes many earth materials to weathering, and the physicalchemical breakdown of some materials are thereby accelerated. All 


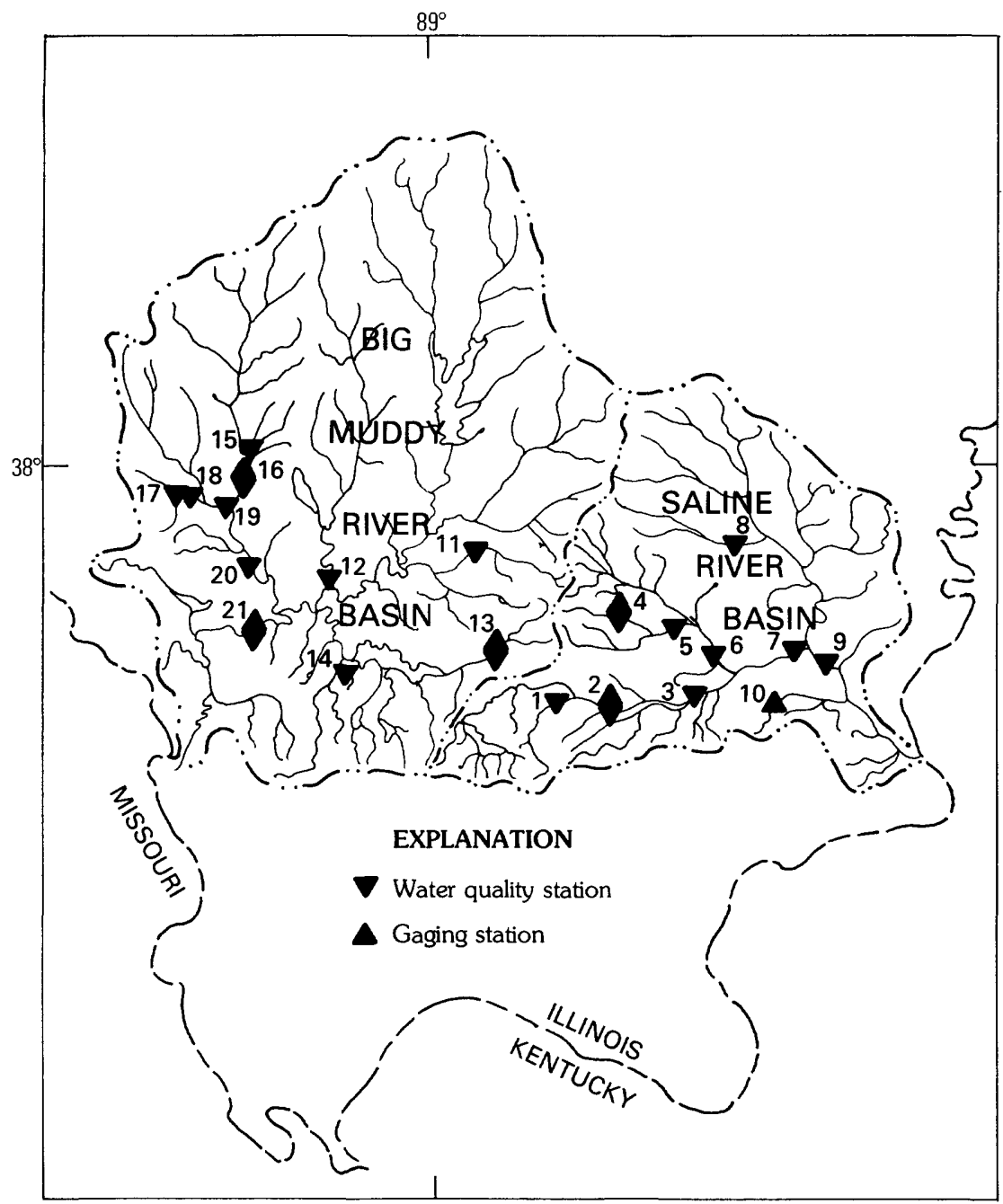

FIGURE 4.-Location of and number identifying sampling sites in Big Muddy and Saline River basins, Illinois.

the reactions are not fully understood (Doyle, 1976, p. 142), but it is generally accepted that water and oxygen act upon iron sulfide to produce ferrous sulfate and sulfuric acid in solution. The ferrous sulfate may react further to produce ferric sulfate and more sulfuric acid. The result is acidic water with high concentrations of sulfate.

Secondary reactions of the acidic water bring many other constituents into solution. These include many metals, such as aluminum, iron, manganese, arsenic, cadmium, mercury, and zinc. These metals are then subject to other reactions such as the formation of precipitates or sorption on sediments. 
If the natural water is sufficiently alkaline, the acidic water may persist only for a short time before neutralization. However, neutralization does not change the concentration of sulfate, and sulfate persists as an indicator of mine drainage. Metals are usually not a reliable indicator of the amount of acidic mine drainage because they may not remain in solution.

The concentration of sulfate in streams depends on the amount produced at some source and the subsequent dilution in the streams. Dilution depends on the discharge of a stream, which varies with several factors such as the amount of precipitation and the drainage area. Concentrations cannot always be compared from stream to stream as an index of mining effect because of these variables. However, the amount of sulfate in acidic mine drainage and the resultant sulfate load in the stream would be expected to vary with the area of the mined land. This assumption was tested with data from the intensive sampling sites.

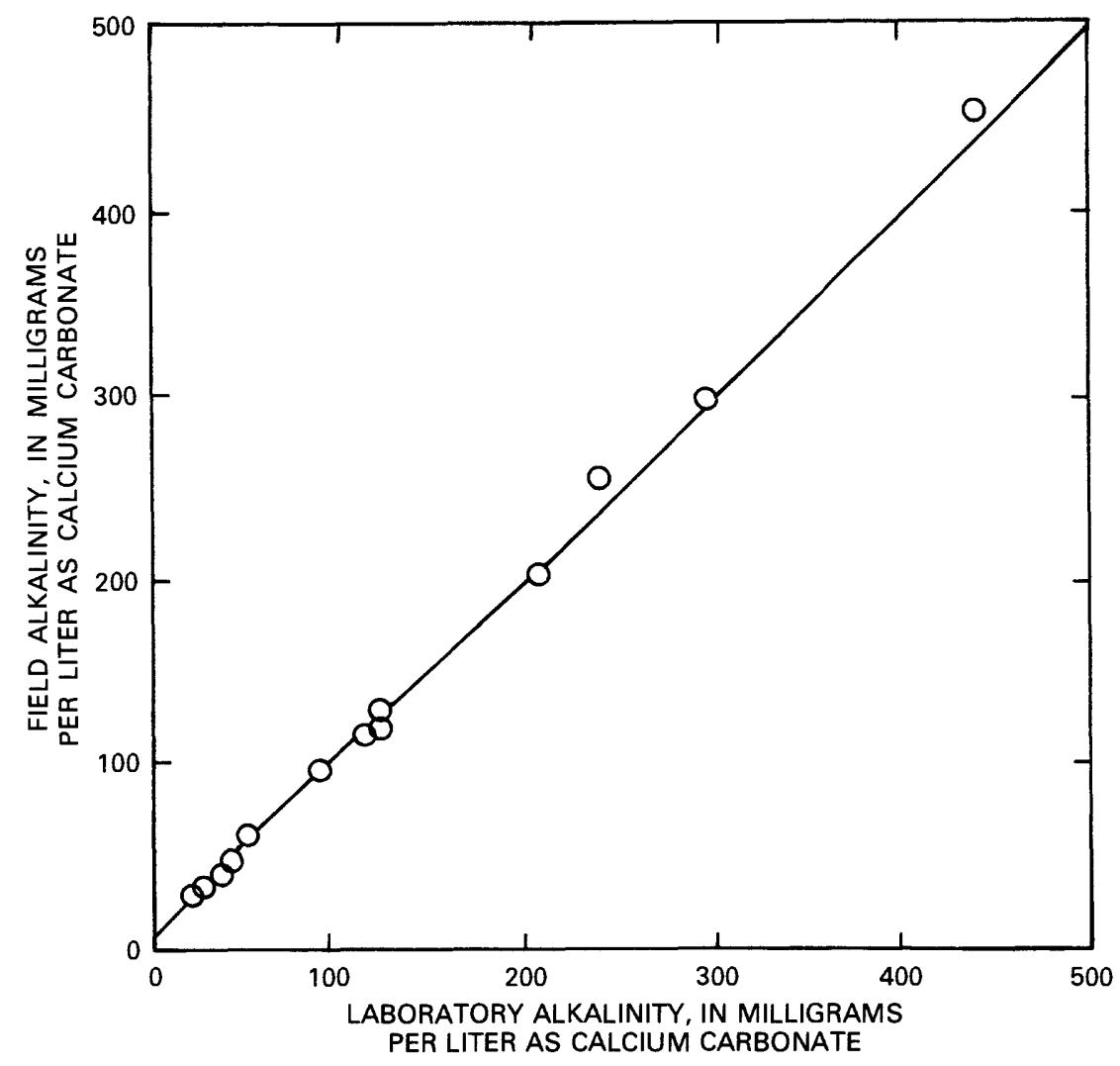

FIGURE 5.-Field and laboratory alkalinity measurements. 


\section{SULFATE LOADS}

Continuous records of both sulfate concentration and stream discharge are needed to compute sulfate loads for any period. Continuous records of both were not available for any sampling site; however, enough data were available for some sampling sites to use the duration curve technique (Searcy, 1959, p. 29) to estimate annual sulfate loads. This technique depends on the correlation of either sulfate concentration or load with stream discharge and the availability of flow duration curves.

Sufficient data to regress sulfate concentrations on concurrent discharge measurements were available for 14 of the 20 sites sampled during the 1976 and 1977 water years. Computerized regression programs were used to relate log-instantaneous discharge to log-sulfate concentration and to log-instantaneous sulfate load. The discharge per square mile was used in the regressions for sulfate load to facilitate the use of the relationships in the duration curve technique for computing average annual sulfate loads. The standards errors (table 1), given in logarithmic deviations and in average percent deviations from the regression line, apply to both equations.

TABLE 1.-Station data for sulfate-concentration and sulfate-load computations $[Q=$ discharge in cubic foot per second; $\boldsymbol{A}=$ drainage area in square miles; $C C=$ Correlation coefficient $]$

\begin{tabular}{|c|c|c|c|c|c|c|c|c|}
\hline \multirow{2}{*}{$\underset{\text { Map }}{\text { number }}$} & \multirow{2}{*}{$\begin{array}{l}\text { Station } \\
\text { number }\end{array}$} & \multicolumn{2}{|c|}{ Sulfate concentration } & \multicolumn{2}{|c|}{ Sulfate load } & \multicolumn{2}{|c|}{ Standard error } & \multirow{2}{*}{$\begin{array}{l}\text { Number of } \\
\text { measurements }\end{array}$} \\
\hline & & Equation & $\mathrm{CC}$ & Equation & $\mathrm{CC}$ & Log & Percent & \\
\hline $1 \ldots$ & 03382090 & $1,400 Q^{-0.543}$ & -0.86 & $19.2(Q / A)^{0.457}$ & 0.81 & 0.203 & 48 & 21 \\
\hline 2 & 03382100 & $2,180 Q^{-0.419}$ & -0.86 & $107(Q / A)^{0.581}$ & 0.92 & 0.195 & 46 & 25 \\
\hline $3-\ldots$ & 03382130 & $1,810 Q^{-0.339}$ & -0.73 & $187(Q / A)^{0.661}$ & 0.90 & 0.234 & 56 & 20 \\
\hline $4 \ldots$ & 03382170 & $162 Q^{0.020}$ & 0.11 & $6.11(Q / A)^{1.02}$ & 0.99 & 0.157 & 37 & 6 \\
\hline $5-$ & 03382185 & $1,640 Q^{-0.133}$ & -0.60 & $194(Q / A)^{0.866}$ & 0.98 & 0.133 & 27 & 20 \\
\hline $8-$ & 03382320 & $196 Q^{-0.056}$ & -0.28 & $31.6(Q / A)^{0.944}$ & 0.98 & 0.200 & 48 & 19 \\
\hline $11-\ldots$ & 05597040 & $176 Q^{-0.137}$ & -0.49 & $9.72(Q / A)^{0.863}$ & 0.96 & 0.269 & 66 & 8 \\
\hline $12-$ & 05597300 & $665 Q^{-0.272}$ & -0.86 & $109(Q / A)^{0.728}$ & 0.98 & 0.156 & 37 & 8 \\
\hline 13 & 05597500 & $456 Q^{-0.162}$ & -0.49 & $22.3(Q / A)^{0.838}$ & 0.95 & 0.252 & 61 & 17 \\
\hline $15-$ & 05598700 & $3,040 Q^{-0.332}$ & -0.80 & & & & & 8 \\
\hline $16 \ldots$ & 05599000 & $3,620 Q^{-0.505}$ & -0.81 & $162(Q / A)^{0.495}$ & 0.80 & 0.247 & 60 & 16 \\
\hline 18 & 05599080 & $1,330 Q^{-0.130}$ & -0.94 & $65.7(Q / A)^{0.760}$ & 0.99 & 0.127 & 30 & 8 \\
\hline 19 & 05599100 & $3,290 A^{-0.423}$ & -0.81 & $167(Q / A)^{0.577}$ & 0.88 & 0.229 & 55 & 16 \\
\hline $21 \ldots$ & 05599500 & $1,350 Q^{-0.287}$ & -0.75 & $869(Q / A)^{0.713}$ & 0.94 & 0.146 & 34 & 49 \\
\hline
\end{tabular}

Sites 6,9 , and 20 had five or fewer measurements and were considered unsuitable for regression analyses. The measurements covered a wide range of discharge and sulfate concentration; therefore, visually estimated lines of relationship were used for these three sites. Sites 7, 14, and 17 were omitted from the load computations because no relationships between discharge and concentration of sulfate load were apparent. 
Continuous discharge records were available for sites $2,4,10,13$, 16, and 21 (fig. 4). All gaged sites except site 10 were also sampling sites. Flow-duration curves were prepared from gaging station records for five gaged sites. These six gaging stations were also used to estimate flow-duration curves for the other sampling sites. Two general curves were prepared, one for sites with drainage areas less than $100 \mathrm{mi}^{2}$ (fig. 6) and one for sites with drainage areas greater than $100 \mathrm{mi}^{2}$ (fig. 7). The flow duration curves were adjusted to discharge per square mile of drainage area to facilitate use of the curves with different drainage areas. The generalized flow duration curves in figures 6 and 7 were visually placed in an intermediate position relative to those for the gaged sites.

The five flow-duration curves prepared from station records and the general curves from figures 6 and 7 were used with sulfate load-discharge relationships for the appropriate gaged and ungaged sites to estimate annual sulfate loads.

The procedure for estimating annual sulfate loads was to first select percent times and corresponding discharges from the duration curves to prepare a flow-duration table. A sulfate-load-duration table was then prepared by determining the sulfate load associated with each discharge value in the flow-duration table. The sulfate loads were determined from the relationships to discharge in table 1 or from the graphical relationships for those sites where regression equations were not available. The product of each time interval and the average sulfate load for the time interval was then summed to obtain the estimated daily sulfate load. Table 2 gives an example of the procedure and the values obtained for one sampling site.

The sulfate loads calculated for 16 sites were regressed against the area of mined land (table 3) upstream from the sampling site. The drainage area and the mined area upstream from site 15 (fig. 4) would require extensive fieldwork to be defined because of modification of the basin divide during mining. This site and the three sites where relationships between discharge and concentration of sulfate were indeterminate were not used.

The relationships of annual sulfate loads to the area of surfacemined land (fig. 8) and the average annual sulfate load per square mile to the percent of surface-mined land (fig. 9) both show that sulfate loads are strongly dependent on the amount of land disturbed by surface mining. The regression coefficients indicate that $3,000-4,000$ tons of sulfate annually enter the streams per square mile of mined land. 


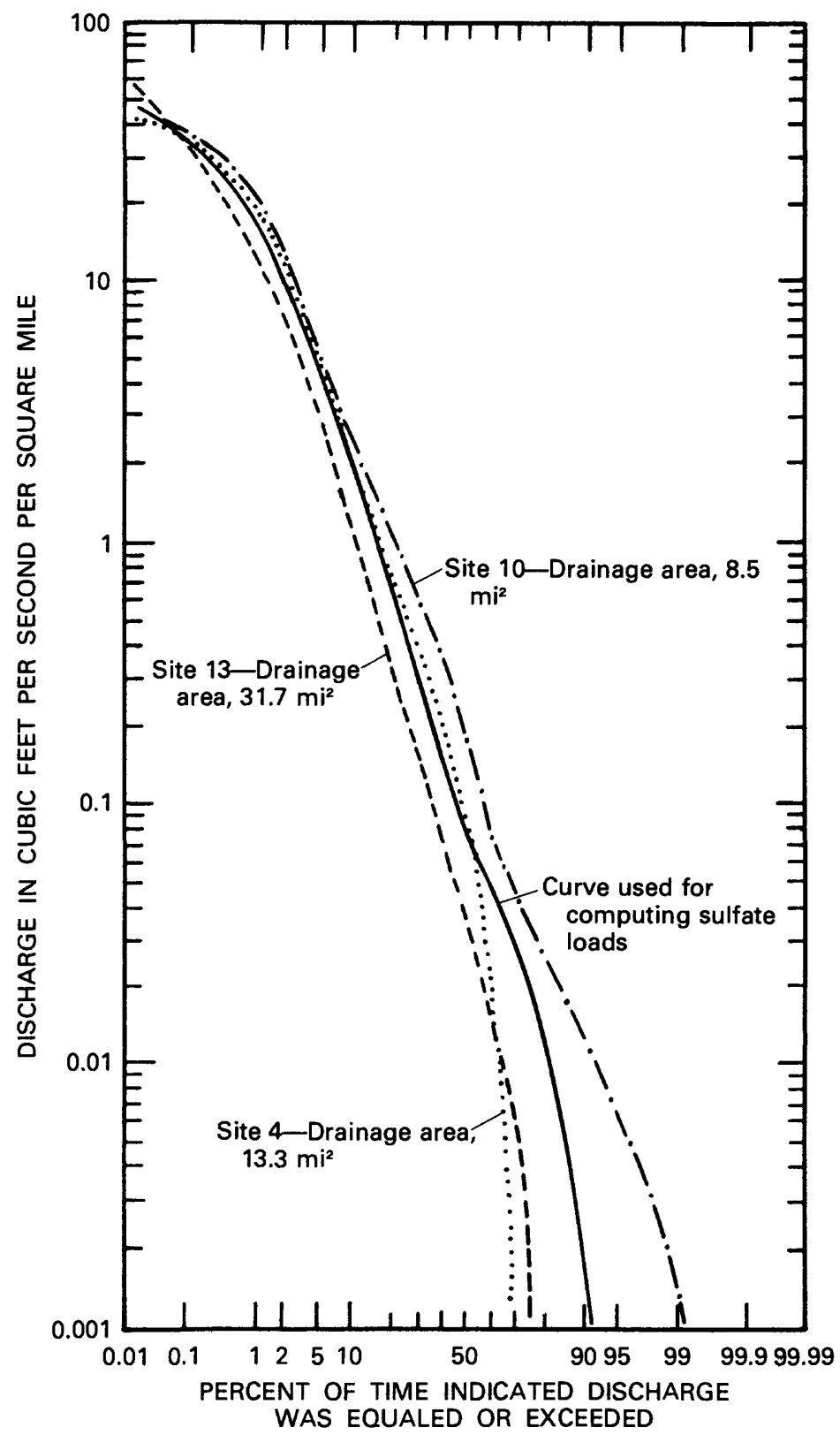

FIgURE 6.-Generalized flow-duration curve for sites less than $100 \mathrm{mi}^{2}$ in the Big Muddy and Saline River basins, Illinois. Location of sites shown in figure 4. 


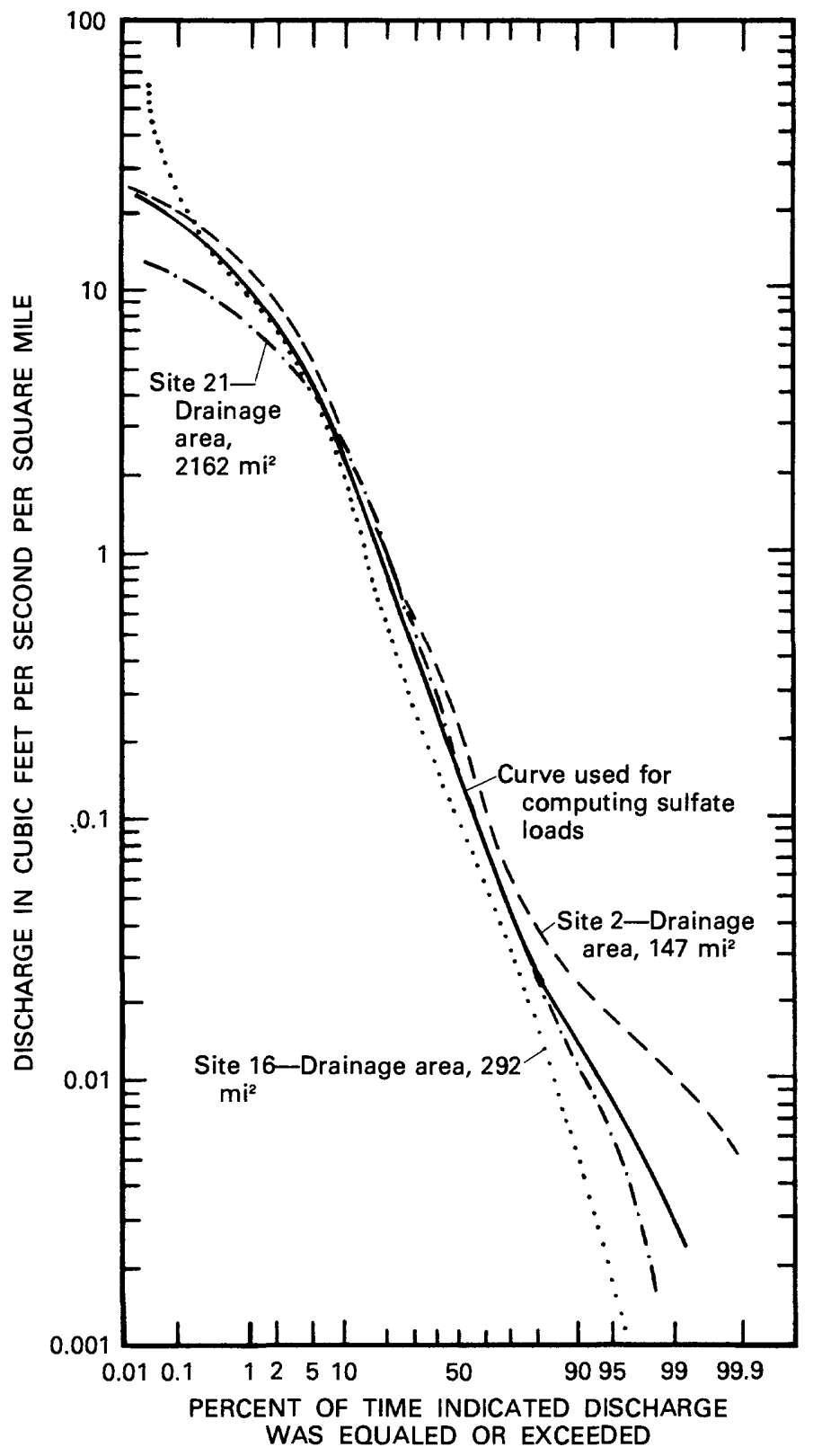

FIGURE 7.-Generalized flow-duration curve for sites greater than $100 \mathrm{mi}^{2}$ in the Big Muddy and Saline River basins, Illinois. Location of sites shown in figure 4. 
TABLE 2.-Tabulation of average annual sulfate load for site 21 by use of the flow-duration curve technique

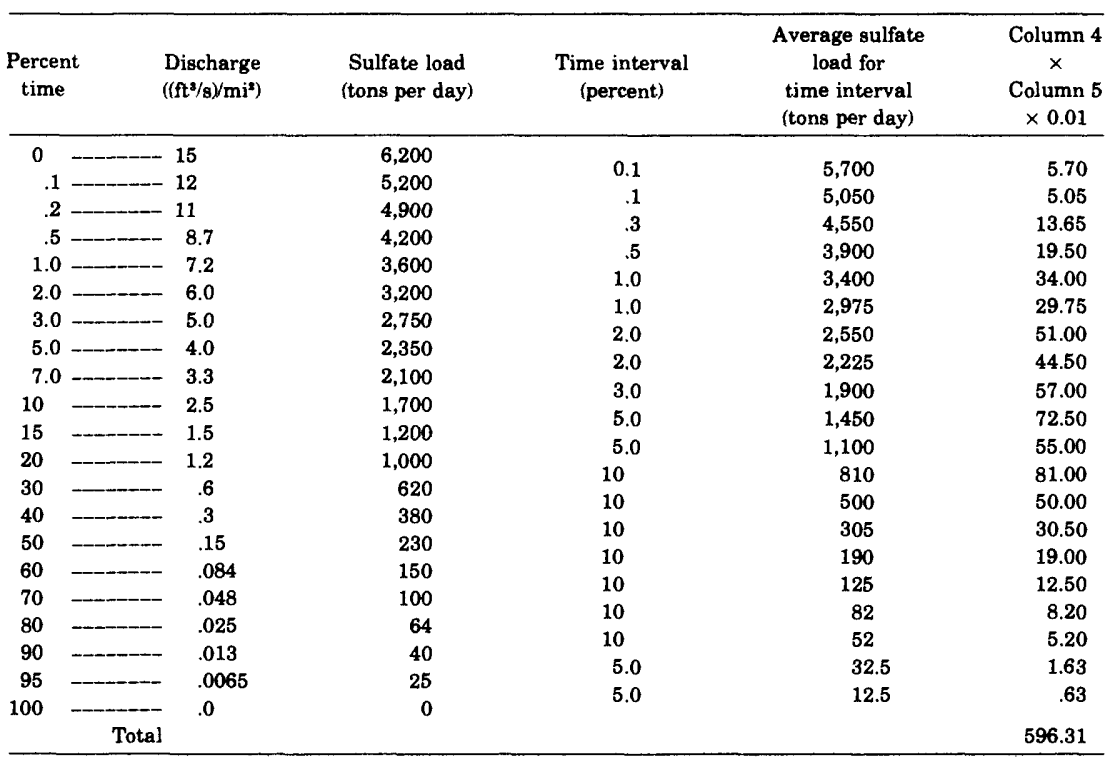

Average tons per day (596.31) times 365 days/yr equals 217,653 tons/yr. Tons per year $(217,653)$ divided by drainage area $\left(2,162 \mathrm{mi}^{2}\right)$ equals 101 tons/yr/mis.

TABLE 3.-Drainage areas, mined areas, and sulfate loads

\begin{tabular}{|c|c|c|c|c|c|c|}
\hline \multirow{2}{*}{$\begin{array}{c}\text { Map } \\
\text { number }\end{array}$} & \multirow{2}{*}{$\begin{array}{l}\text { Station } \\
\text { number }\end{array}$} & \multirow{2}{*}{$\begin{array}{l}\text { Drainage area } \\
\text { (square miles) }\end{array}$} & \multicolumn{2}{|c|}{ Mined area } & \multicolumn{2}{|c|}{ Annual average sulfate load } \\
\hline & & & $\begin{array}{c}\text { Square } \\
\text { miles }\end{array}$ & Percent & Tons & $\begin{array}{c}\text { Tons per } \\
\text { square mile }\end{array}$ \\
\hline $11 \ldots$ & 05597040 & 33.1 & 0.0 & 0.0 & 2,970 & 90 \\
\hline 8- & 03382320 & 76.4 & .0 & .0 & 10,800 & 141 \\
\hline 4-_- & 103382170 & 13.3 & .1 & .8 & 2,410 & 181 \\
\hline $21 \ldots$ & ${ }^{2} 05599500$ & 2,162 & 51.6 & 2.4 & 218,000 & 101 \\
\hline $12-$ & 05597300 & 283 & 6.7 & 2.4 & 27,200 & 96 \\
\hline $1-\ldots$ & 03382090 & 35.4 & 1.1 & 3.1 & 4,200 & 119 \\
\hline 9 & 203382450 & 1,022 & 31.3 & 3.1 & 119,000 & 116 \\
\hline 13 ב_ב_ & ${ }^{1} 05597500$ & 31.7 & 1.2 & 3.8 & 4,640 & 146 \\
\hline $3 \ldots$ & 03382130 & 248 & 12.1 & 4.9 & 45,400 & 183 \\
\hline $20 \ldots$ & 205599300 & 516 & 25.5 & 4.9 & 96,500 & 187 \\
\hline $2-\cdots$ & ${ }^{1} 03382100$ & 147 & 9.8 & 6.7 & 29,000 & 197 \\
\hline $6-\ldots$ & ${ }^{2} 03382205$ & 233 & 15.8 & 6.8 & 52,600 & 226 \\
\hline 19 & 05599100 & 162 & 12.1 & 7.5 & 39,800 & 246 \\
\hline 16 & ${ }^{1} 05599000$ & 292 & 24.1 & 8.3 & 33,600 & 115 \\
\hline $18-$ & 05599080 & 46.1 & 5.5 & 11.9 & 16,400 & 356 \\
\hline 5-_-_-_ & 03382185 & 77.9 & 14.8 & 19.0 & 58,940 & 757 \\
\hline
\end{tabular}

1 Gaging station.

'Insufficient data for regression analysis; eye-fit line used for load calculation. 


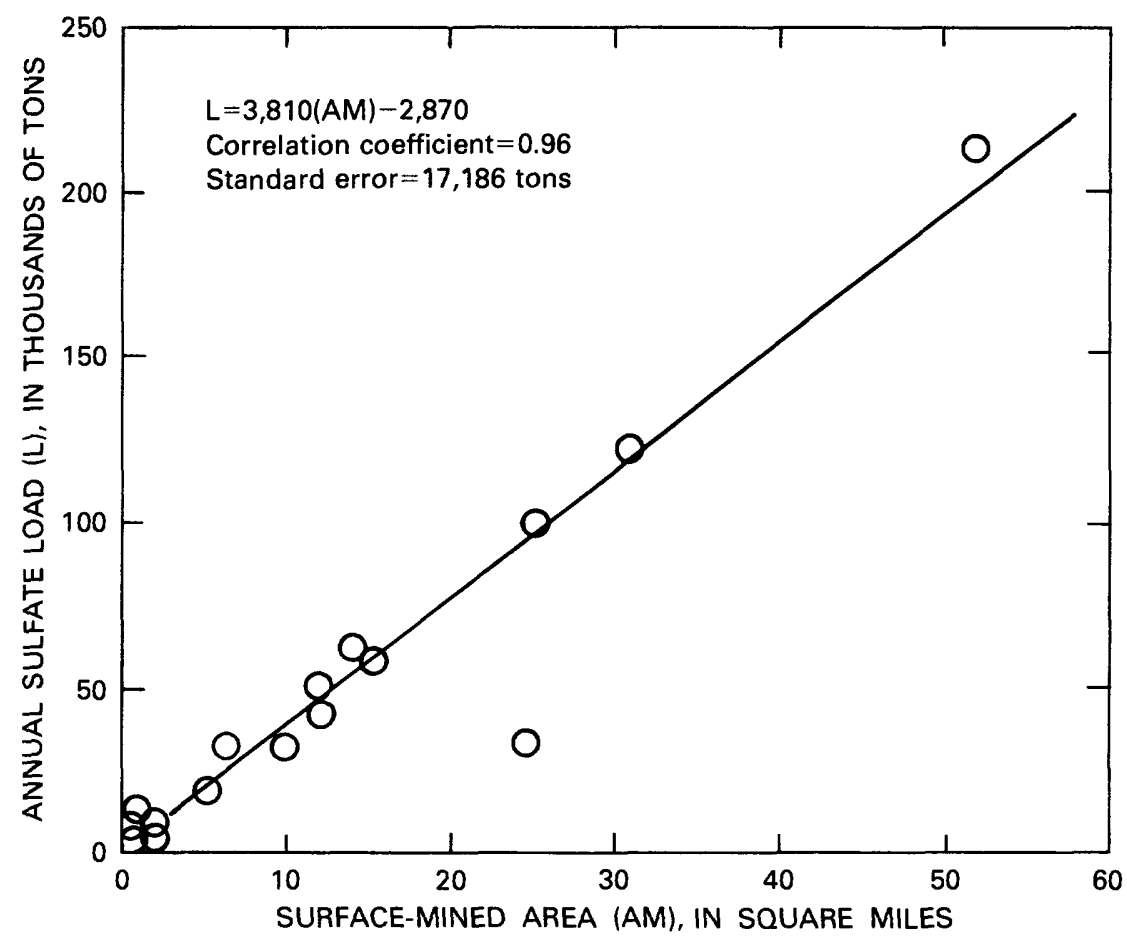

FIGURE 8.-Relationship of annual sulfate load to area of surface-mined land.

\section{DATA NEEDS}

The data collected from 1975 to 1977 provide considerable information on chemical characteristics of streams in surface-mined areas in Illinois and suggest that additional data are needed to define better the hydrology.

The concentrations of dissolved solids, sulfate, and many metals are relatively high near the mine sites, where the water is commonly acidic. In larger streams, acidic water from mine drainage is readily neutralized, and concentration of the above constituents are lower. The inverse correlation of discharge to sulfate concentration (table 1) suggests that dilution is the principal cause of attenuation of sulfate.

The dissolved concentrations of most of the major constituents are directly related to each other and to specific conductance. Figure 10 shows two relationships for one station and indicates that measurements of specific conductance could be used to estimate accurately concentrations of some constituents. As specific conductance is readily measurable, these relationships could be used with recorded flow data to calculate, with a fair degree of accuracy, the dissolved loads of certain constituents contributed by mined areas. 


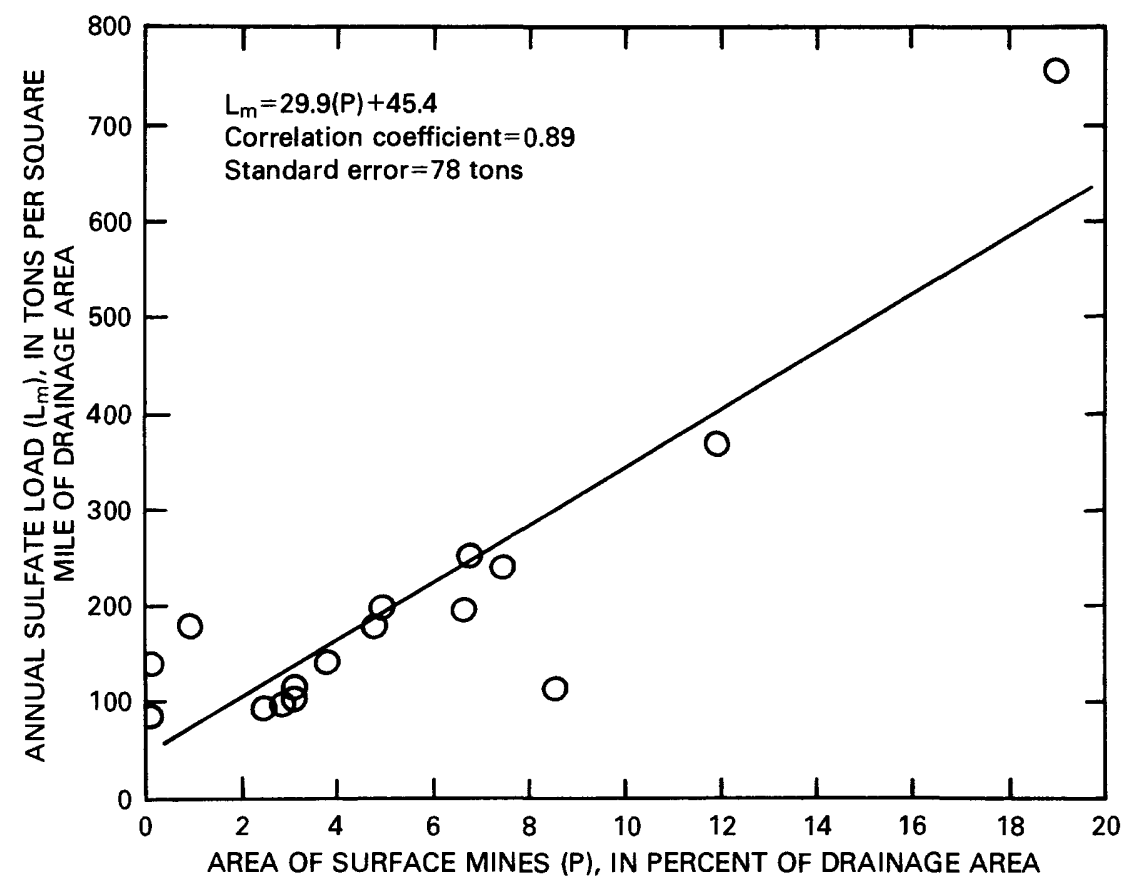

Figure 9.-Relationship of annual sulfate load per square mile of drainage area to percentage of surface-mined land.

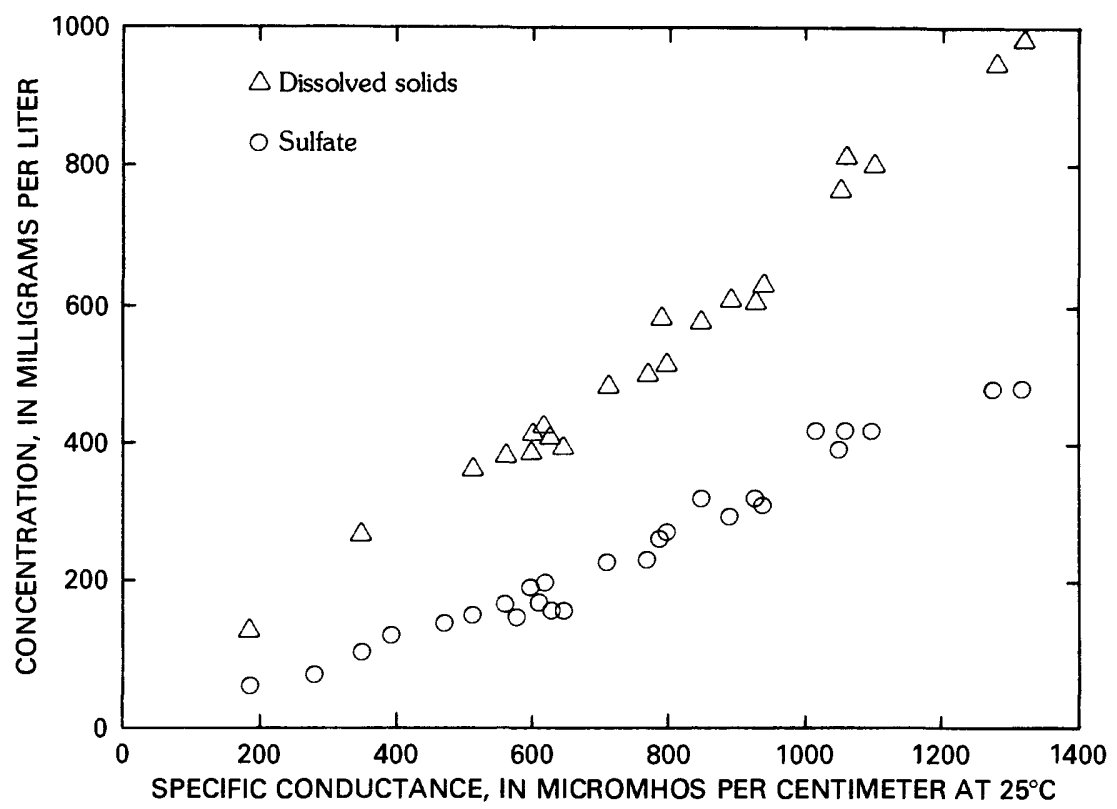

FIGURE 10.-Relationship of specific conductance to dissolved solids and sulfate concentrations for Big Muady River at Murphysboro, Illinois. 
Although many of the major constituents are inversely related to discharge, which suggests that attenuation is largely by dilution, no well-defined relationships were observed for the minor constituents. Metals, in particular, are considered susceptible to adsorption and precipitation, but data are not available to evaluate whether any constituents are being concentrated in bottom materials or how metals absorbed on sediments are transported.

Data are also needed to evaluate the changes in runoff caused by surface mining in Illinois. Corbett (1966) concluded that floodflows in one watershed in southern Indiana were materially reduced by "cast overburden". Comparison studies of runoff from mined areas with that from nearby unmined areas would provide the information needed for this evaluation.

\section{SUMMARY}

Bituminous coal reserves in Illinois make up a large part of the Eastern Interior Coal Field. On the periphery of a structural basin in Illinois, the coal is amenable to removal by surface mining. As of June 1974, 181,000 acres had been affected by surface mining.

The water quality in streams has been altered by runoff from the mined areas. Analyses of stream water made from 1975 to 1977 show that acidic water is formed in many mined areas but is usually neutralized before it reaches the larger streams. Sulfate loadings in streams in southern Illinois are directly related to the area of mined land in the basin. Annually, 3,000-4,000 tons of sulfate per square mile of mined land enter the streams.

High sulfate concentrations are associated with high concentrations of dissolved solids. Concentrations of aluminum, arsenic, chromium, copper, iron, manganese, and zinc are significant in the acidic waters.

\section{REFERENCES CITED}

Brown, Eugene, Skougstad, M. W., and Fishman, M. J., 1970, Methods for collection and analysis of water samples for dissolved minerals and gases: U.S. Geological Survey Techniques of Water-Resources Investigations, Book 5, Chapter A1, $160 \mathrm{p}$.

Corbett, D. M., 1966, Surface mining for coal-Effect on storm runoff: Water Resources Research Center, Indiana University, first interim report, $19 \mathrm{p}$.

Doyle, W. S., 1976, Strip mining of coal, environmental solutions: Noyes Data Corporation, Park, Ridge, N.J., 307 p.

Greater Egypt Regional Planning and Development Commission and Southeastern Illinois Regional Planning and Development Commission, 1978, Southern Illinois 208 mine waste control program, Volume 3, map appendix section, map 5, location of surface mines, scale, one inch equals two miles.

Haynes, R. J., and Klimstra, W. D., 1975, Some properties of coal spoil bank and refuse materials resulting from surface-mining coal in Illinois: Illinois Institute of Environmental Quality Document 75-21, 126 p. 
Office of Water Resources Research, 1975, Acid mine water, a bibliography: U.S. Department of Commerce, National Technical Information Service PB-239 523, $563 \mathrm{p}$.

Searcy, J. K., 1959, Flow-duration curves: U.S. Geological Survey Water-Supply Paper 1542-A, 33 p.

Smith, W. H., and Stall, J. B., 1975, Coal and water resources for coal conversion in Illinois: Illinois State Geological Survey and Illinois State Water Survey Cooperative Resources Report 4, 79 p., 3 pls. 
TABLES 4,5 
Table 4.--Chemical analyses of samples collected at reconnaissance sites

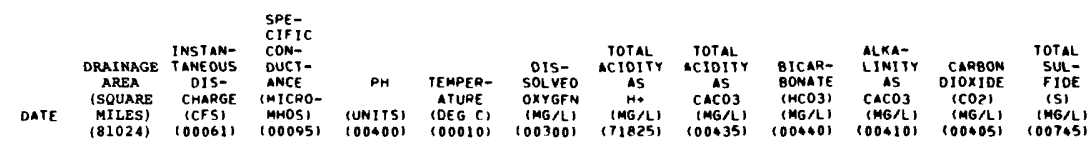

03338090 - JOROAN CREEK AT FAIRMOUNT, IL (LAT 40 O3 26 LONG OBT SO 111

\begin{tabular}{|c|c|c|c|c|c|c|c|c|c|c|c|c|}
\hline${ }_{\operatorname{MAN}}^{12 . .}$ & 517.8 & 14 & 643 & 7.9 & $14 . n$ & 11.2 & .0 & .0 & 260 & 213 & 5.2 & .0 \\
\hline $19 .$. & 17.8 & 13 & 660 & 7.4 & 22.5 & 7.7 & .0 & .0 & 278 & 228 & 18 & 1.4 \\
\hline & & & 033390 & GRAPE & REEK & ILTON. & ILAT & 42 & 087 & 8) & & \\
\hline MaY $12 .$. & ${ }^{5} 11.8$ & 7.3 & 818 & 7.3 & 12.0 & 9.2 & .0 & .0 & 244 & 200 & 20 & .0 \\
\hline $19 \ldots$ & 11.8 & 11 & 770 & 6.8 & 20.0 & B.2 & .0 & .0 & 246 & 202 & 62 & .0 \\
\hline
\end{tabular}

03339142 - FAYETTE DRAIN NEAR INOIANOLA, IL ILAT 3957 O5 LONG 08742301

\begin{tabular}{|c|c|c|c|c|c|c|c|c|c|c|c|}
\hline${ }_{j, N}$ & ${ }^{5} 10.3$ & 12 & 666 & 7.9 & 13.0 & 11.5 & .0 & .0 & 276 & 226 & 5.6 \\
\hline $19 .$. & 10.3 & 11 & 752 & 7.3 & 20.5 & 9.4 & .0 & .0 & 293 & 240 & 23 \\
\hline
\end{tabular}

03346150 - BIG CREEK NEAR OBLONG. IL ILAT 390012 LONG 08750281

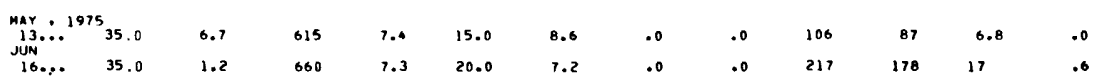

03346180 - OOGHOOD CREEK AT OQLONG. IL LLAT 39 OO O7 LONG 0875338 I

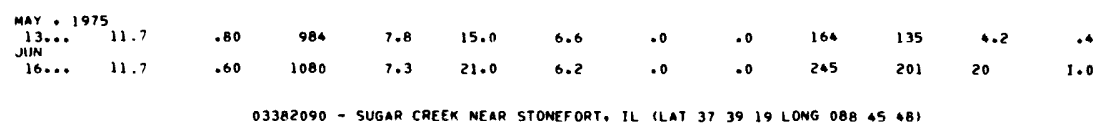

\begin{tabular}{|c|c|c|c|c|c|c|c|c|c|c|c|c|}
\hline נדו.... & 35.4 & 5.0 & 760 & 3.6 & 16.5 & 7.7 & 5.5 & 273 & 0 & 0 & .0 & .5 \\
\hline $17 \ldots$ & 35.4 & EA.1 & 2420 & 3.3 & 23.0 & .3 & 44 & 2180 & 0 & 0 & .0 & 1.0 \\
\hline
\end{tabular}

03382120 - LITILE SALINE RIVER NEAR STONEFORT, IL (LAT 3735 O4 LONG $08 B 40091$

\begin{tabular}{|c|c|c|c|c|c|c|c|c|c|c|c|c|}
\hline JUN & 19.5 & 13 & 120 & 6.9 & 15.0 & A. 8 & .0 & .0 & 23 & 19 & 4.6 & .0 \\
\hline $17 \ldots$ & 19.5 & .50 & 167 & 6.9 & 21.0 & 5.5 & .0 & .0 & $6 B$ & 56 & 14 & 1.4 \\
\hline
\end{tabular}

03382155 - PRAIRIE CREEK MEAR CORINTH. IL ILAT 37 49 37 LONG 68842361

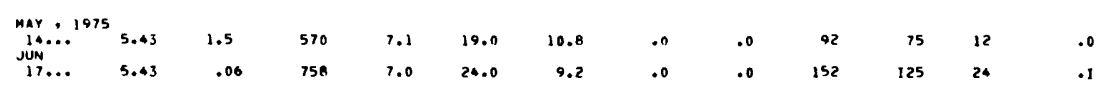

03382175 - bRUSHY CREEK TRIO NEAR MARCO, IL ILAT 37 46 33 LONG 08837091

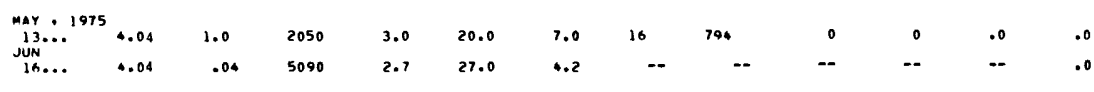


Table 4.--Chemical analyses of samples collected at reconnaissance sites--Continued

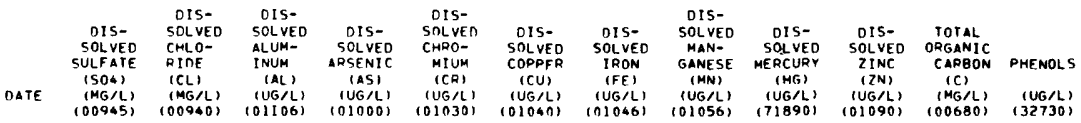

03338090 - JORDAN CREEK AT FAIRMOUNT, IL ILAT 400326 LONG 08750111

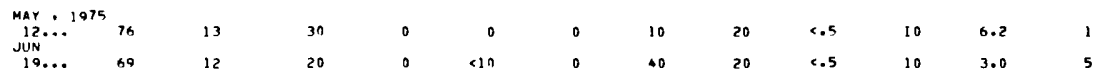

03339040 - GRAPE CPEEK AT TILTON. IL ILAT 40 OS 42 LONG 087 38 08)

\begin{tabular}{|c|c|c|c|c|c|c|c|c|c|c|c|}
\hline 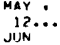 & ${ }^{5} 120$ & 26 & 70 & 1 & 0 & 0 & 30 & 290 & .6 & 1100 & 5.7 \\
\hline $19 \ldots$ & 110 & 22 & so & 0 & 0 & 0 & 20 & 260 & c.5 & 690 & 19 \\
\hline
\end{tabular}

03339142 - FAYETTE ORAIN NEAR INDIANOLA. IL (LAT 3957 O5 LONG 087 .2 30)

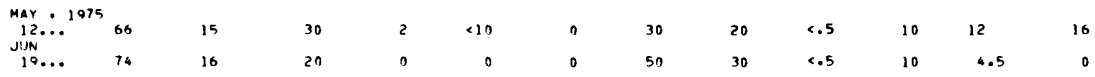

03345150 - BIG CREEK NEAR OALONG. IL (LAT 390012 LONG OQ7 50 28)

\begin{tabular}{|c|c|c|c|c|c|c|c|c|c|c|c|}
\hline JUN $13 \ldots$ & 33 & 89 & 180 & 1 & 10 & 10 & 260 & 120 & .8 & 390 & \\
\hline $16 \ldots$ & 25 & $9 n$ & 20 & 1 & $<10$ & 0 & 60 & 490 & 1.3 & 0 & 9.6 \\
\hline
\end{tabular}

03346180 - DOGWOOO CREEK AT OBLONG. IL (LAT 39 OO OT LONG OAT 5338 )

\begin{tabular}{|c|c|c|c|c|c|c|c|c|c|c|c|}
\hline JUN & 50 & $1 A_{0}$ & 110 & 2 & 20 & 20 & 180 & 500 & .7 & 60 & 13 \\
\hline $16 \ldots$ & 63 & 200 & 40 & $\mathbf{s}$ & $1 n$ & 10 & 70 & 1000 & .6 & 10 & 18 \\
\hline
\end{tabular}

033P2090 - SUGAR CREEK NEAR STONEFORT. IL ILAT 373919 LONG OB8 45 48)

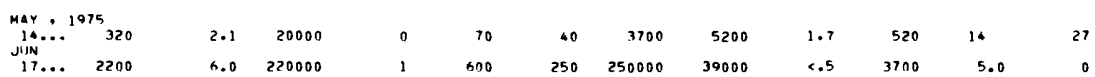

03382120 - LITTLE SALINE RIVER NEAR STONFFORT. IL ILAT 3735 O4 LONG 08B 60 09)

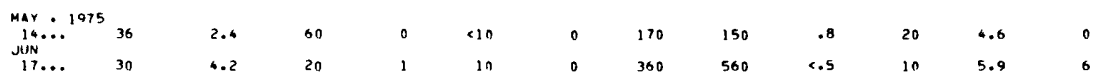

03382155 - PRAIRIE CAEEK NEAR CORINTH. IL ILAT 374937 LONG 08842361

\begin{tabular}{|c|c|c|c|c|c|c|c|c|c|c|c|}
\hline JUN & $16 n$ & 16 & 210 & 0 & $n$ & 10 & 260 & 60 & 1.1 & 20 & 4.0 \\
\hline $17 \ldots$ & 180 & 68 & 220 & 1 & 20 & 10 & 110 & 90 & .7 & 0 & 5.2 \\
\hline
\end{tabular}

0338217S - RRUSHY CREEK TRIB NEAR HARCOV IL ILAT $3746 \quad 33$ LONG 08837 09)

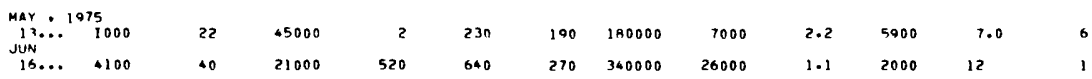


Table 4,--Chemical analyses of samples collected at reconnaissance sites--Continued

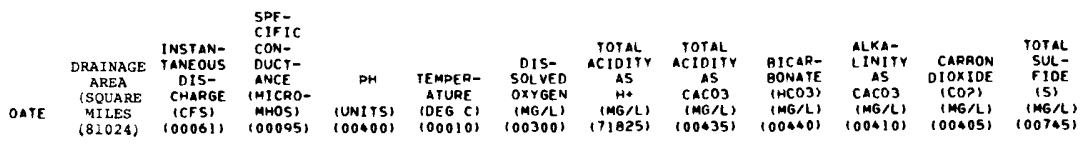
O338ZIAO - BANKSTON FORK AT DORRIS HEIGHTS, IL ILAT 374534 LONG OBB 36101

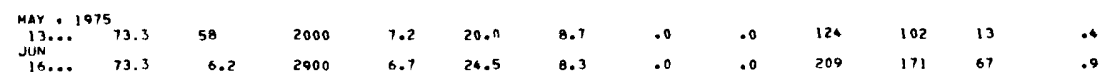

OJ3R2ZIN - ARIER CREEX NEAR HARRISBURG. IL ILAT 37 AI 60 LONG 08832101

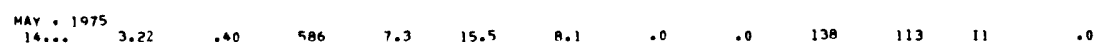
05447370 - MUD CREEK AT ANNAWAN. IL ILAT 41 24 O4 LONG O89 55131

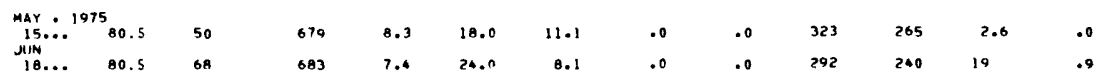

OS447390 - COAL CREEK AT SHEFFIELD, IL ILAT 41 21 40 LONG 089 \$4 \$31

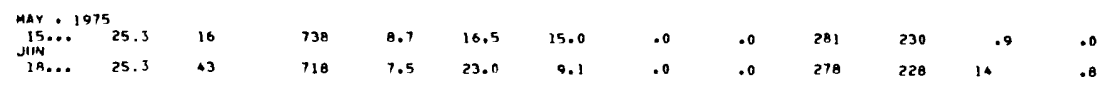

05447400 - KING CREEK AT MINERAL. IL LLAT 412300 LONG OBg 50 34)

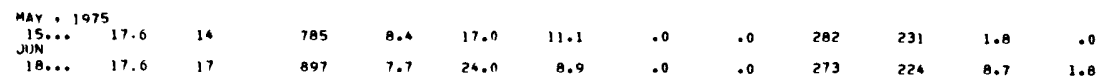

05466010 - MUD CPEEK AT OPHIEM, IL LLAT \&I 1503 LONG 09022401

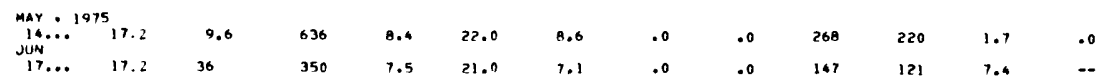

05466600 - POPE CREEK NEAR ALPHA, IL ILAT 41 OQ 45 LONG 09022 4TI

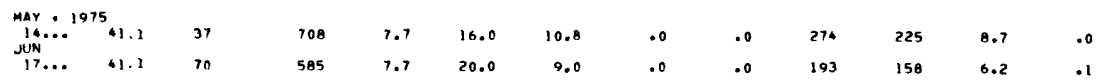

05\$66630 - NORYH POPE CREEK AT NEW WINOSOR. IL ILAT 411130 LONG 09026 47,

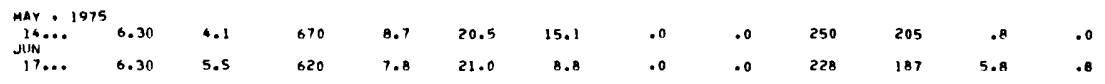

D552T0O2 - HORSE CREEK NEAR ESSEX, IL ILAT AI 10 38 LONG OBA OQ 001

\begin{tabular}{|c|c|c|c|c|c|c|c|c|c|c|c|}
\hline $12 \ldots$ & 107 & 46 & 690 & 8.3 & 16.5 & 14.3 & .0 & .0 & 211 & 173 & 1.7 \\
\hline $19 \ldots$ & 107 & 186 & 103 & 7.2 & 25.5 & 8.5 & .0 & .0 & 233 & 191 & 24 \\
\hline
\end{tabular}


Table 4.--Chemical analyses of samples collected at reconnaissance sites--Continued

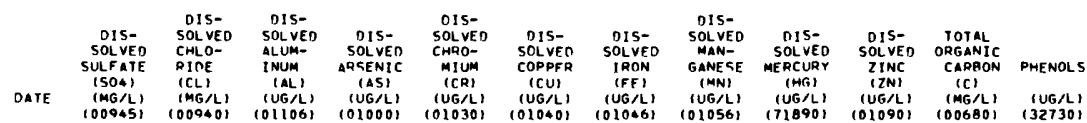

O33A2180 - GANKSTON FORK AT DORRIS HEIGMTS, IL (LAT 37 45 34 LONG OBB 34101

\begin{tabular}{|c|c|c|c|c|c|c|c|c|c|c|c|}
\hline & $\begin{array}{l}1100 \\
1900\end{array}$ & 16 & 20 & 1 & 10 & 10 & 20 & & $\begin{array}{r}.6 \\
<.5\end{array}$ & 0 & 4.5 \\
\hline $16 \ldots$ & 1900 & 23 & 110 & 0 & 20 & 20 & 30 & 640 & $<.5$ & 40 & 16 \\
\hline
\end{tabular}

03382210 - BRIER CREEK NEAR HARRISGURG, IL ILAT 37 41 40 LONG 08832101

MAY 1975 80

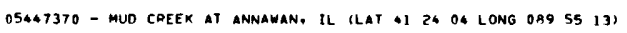

MAY 1975
JUN....
IR...

18

30

40

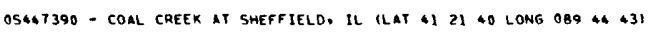

$\max _{\text {JuN }}^{15 . .0^{1975} 100}$ 15

$$
20
$$

$$
20
$$$$
60
$$

\begin{tabular}{|c|c|c|c|c|c|c|c|c|c|c|c|c|}
\hline $15 \ldots$ & 220 & 8.6 & 10 & 1 & $<10$ & 10 & 20 & 70 & .5 & 0 & 1.8 & 4 \\
\hline IR... & 230 & 11 & 50 & 1 & 0 & 0 & 50 & 50 & $<.5$ & 10 & 11 & 3 \\
\hline
\end{tabular}

05447400 - KING CREEK AT MINERAL, IL ILAT 412300 LONG 08950341

\begin{tabular}{|c|c|c|c|c|c|c|c|c|c|c|c|}
\hline JUN..." & 48 & 13 & 20 & 0 & 0 & 0 & 20 & 20 & c.s & 0 & 3.7 \\
\hline $17 \ldots$ & 27 & 14 & 190 & 1 & $n$ & 0 & 160 & 140 & c.5 & 20 & 71 \\
\hline
\end{tabular}

05466010 - MUO CREEK AT OPHIEM, IL ILAT \& 1503 LONG 0902260$)$

05466600 - POPE CREEK NEAR ALPHA, IL ILAT 41 O8 45 LONG 09022 47

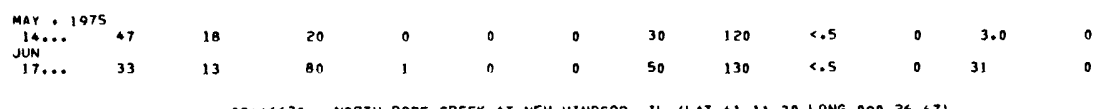

OS466630 - NORIH POPE CREEK AT NEW WINOSOR, IL ILAT 411130 LONG 09026471

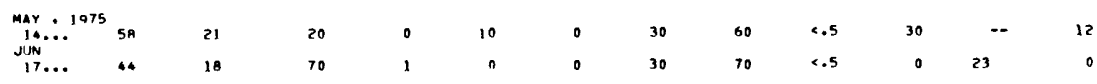

05527002 - HORSE CAEEK NEAR ESSEX. IL ILAT 41 10 38 LONG OB8 09001

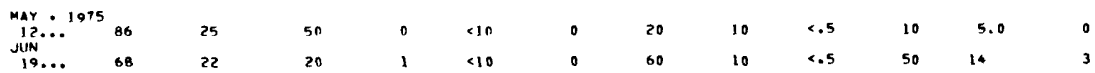


Table 4.--Chemical analyses of samples collected at reconnassance sites--Continued

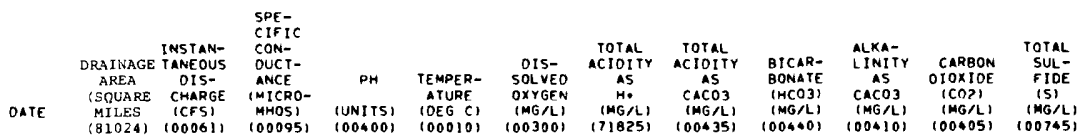

05568450 - LITTLF LAMAPSH CREEK AT MAPLETON. IL (LAT 403346 LONG 0894436 )

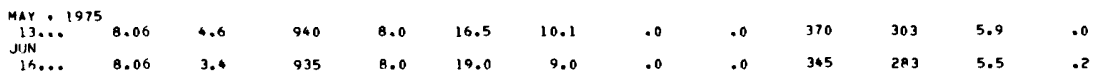

05568770 - SPOON RIVER AT MODENA, IL ILAT 41 OQ OB LONG 0894538 I

\begin{tabular}{|c|c|c|c|c|c|c|c|c|c|c|c|}
\hline$\underset{\text { JuN }}{\operatorname{Mar} . .}$ & 154 & 113 & 732 & & & & .0 & & 279 & 229 & 2.8 \\
\hline $19 \ldots$ & 154 & 248 & 752 & 7.8 & $21 . n$ & 8.4 & .0 & .0 & 273 & 224 & 6.9 \\
\hline
\end{tabular}

05568772 - JACK CREEK AT MOOENA. IL ILAT 41 OB 05 LONG 0894640 )

\begin{tabular}{|c|c|c|c|c|c|c|c|c|c|c|c|c|}
\hline JUN & 15.7 & 12 & 640 & 0.3 & 15.0 & 12.8 & .0 & .0 & 302 & & 2.4 & .0 \\
\hline $19 \ldots$ & 15.7 & $1 \mathrm{~A}$ & 696 & 7.9 & 18.0 & 9.2 & .0 & .0 & 297 & 246 & 6.0 & .0 \\
\hline
\end{tabular}

A5568795 - INOIAN CREEK AT TOULON. IL ILAT 4 I 0555 LONG 0895306 I

\begin{tabular}{|c|c|c|c|c|c|c|c|c|c|c|c|}
\hline JUN & 39.6 & 42 & 768 & 7.9 & 13.0 & & .0 & .0 & 284 & 233 & 5.7 \\
\hline $18 \ldots$ & 39.6 & 61 & 648 & $7 . A$ & 21.5 & 9.1 & . 0 & .0 & 256 & 208 & 6.6 \\
\hline
\end{tabular}

05568860 - WALNUT CREEK NEAR WFST JERSEY. IL ILAT 41 OI 05 LONG 08957431

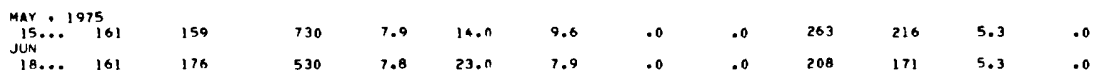

05568895 - SNAKEOEN HOLLOW NEAR OAHINOA. IL ILAT 405734 LONG 090 O4 15,

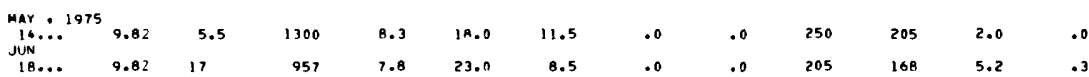

05568910 - SUGAR CREEK AT DAMINOA. IL ILAT 405540 LONG 090 OT 00

\begin{tabular}{|c|c|c|c|c|c|c|c|c|c|c|c|c|}
\hline$\underset{\text { JuN }}{\max } . .$. & 21.6 & 15 & 706 & 8.4 & 19.0 & 9.7 & .0 & .0 & 209 & 171 & 1.3 & $\cdot 1$ \\
\hline $18 \ldots$ & 21.6 & 30 & 742 & 8.1 & 22.0 & B. 2 & .0 & .0 & 195 & 160 & 2.5 & . 3 \\
\hline
\end{tabular}

05568940 - HICKORY CREEK MEAR MAQUON, IL ILAT 404643 LONG 090 O8 OTI

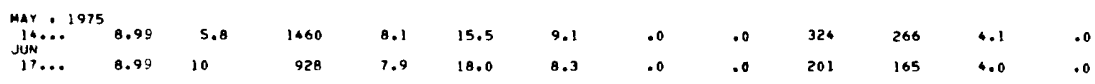

05569450 - LITTLERS CREEK NEAR RAPATEE. IL LLAT 404453 LONG 09009281

\begin{tabular}{|c|c|c|c|c|c|c|c|c|c|c|c|}
\hline $\begin{array}{l}14 \ldots \\
\operatorname{Jun}\end{array}$ & 33.3 & 14 & 1080 & $\begin{array}{l}0.4 \\
7.9\end{array}$ & 16.0 & & $\begin{array}{l}.0 \\
.0\end{array}$ & .0 & 308 & 253 & $\begin{array}{l}2.0 \\
5.2\end{array}$ \\
\hline $17 \ldots$ & 33.3 & 26 & 1110 & 7.9 & 19.0 & 6.4 & $\bullet 0$ & & $25 \mathrm{~B}$ & 212 & 5.2 \\
\hline
\end{tabular}


Table 4.--Chemical analyses of samples collected at reconnaissance sites--Continued

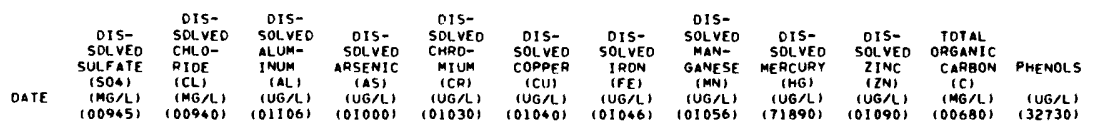

05568450 - LITTLE LAMARSM CREEK AT MAPLETON, IL (LAT 4033 4A LONG 0894436 )

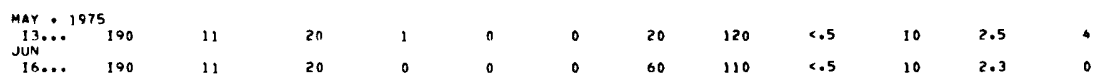

055687TO - SPOON RIVER AT MODENA, IL ILAT 4 I OB OB LONG OB9 4538 I

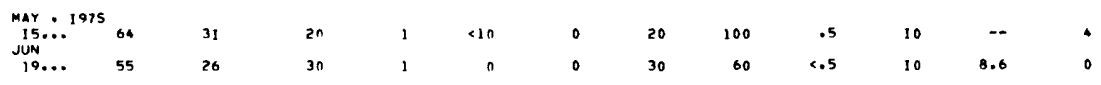

055687T? - JACK CREEK AT MODENA. IL ILAT 4 I OB O5 LONG 0894640 )

\begin{tabular}{|c|c|c|c|c|c|c|c|c|c|c|c|}
\hline $\begin{array}{l}\text { MAY } \\
15 . .1^{1975}\end{array}$ & 56 & is & 20 & 0 & 10 & 10 & 50 & 50 & .5 & 10 & 3.5 \\
\hline $19 . .$. & 47 & 14 & 40 & 1 & 0 & 0 & 10 & 40 & $<.5$ & 0 & 9.8 \\
\hline
\end{tabular}

05568795 - INDIAN CREEK AT TOULON. IL (LAT II OS 55 LONG OB9 53 O6)

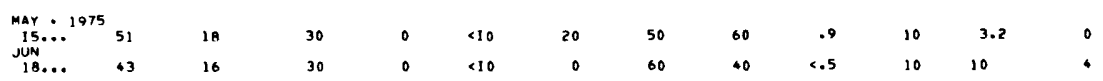

05568B60 - WALNUT CREEK NEAR WEST JEASEY, IL ILAT 4I OI O5 LONG OB9 57 431

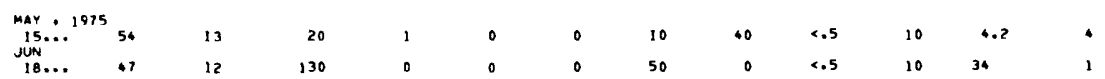

05568895 - SNAKEOEN HOLLOH NEAR OAHINOA. IL ILAT 405734 LONG 09004151

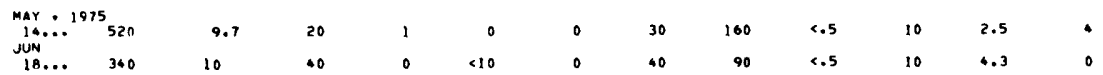

05568910 - SUGAR CREEK AT DAHINOA. IL ILAT 4055 40 LONG 090 OT OOI

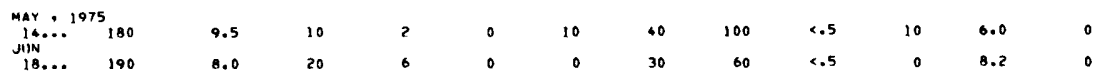

05568940 - HICKORY CREEK NEAR MAOUON. IL ILAT 404643 LONG 090 OB OTI

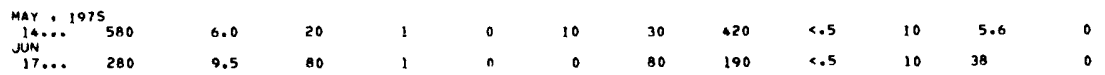

055694SO - LITTLERS CREEK NEAR RAPATEE. IL ILAT 404453 LONG 0900928 )

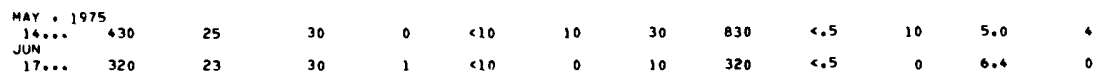


Table 4.--Chemical analyses of samples collected at reconnassance sites--Continued

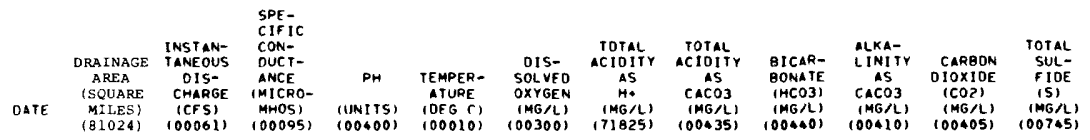

05569920 - COAL CREEK NEAR FAIRVIEW. IL ILAT 403933 LONG 0901400 )

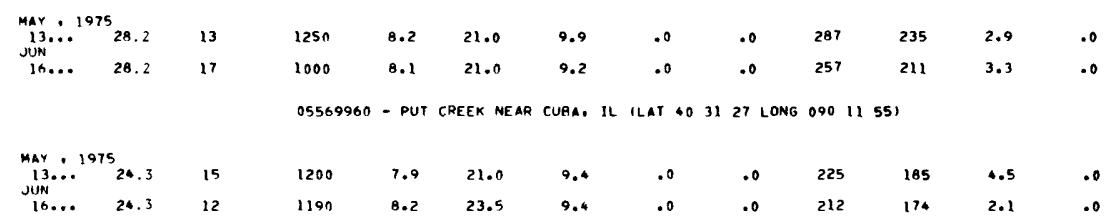

05569970 - TURKEY CREEK NEAR ALYTON, IL ILAT 403326 LONG 09015 401

\begin{tabular}{|c|c|c|c|c|c|c|c|c|c|c|c|c|}
\hline MAY & ${ }^{5} 35.6$ & 14 & 612 & 8.2 & 23.0 & 10.0 & .0 & .0 & 280 & 230 & 2.8 & .2 \\
\hline $\begin{array}{l}\text { JUN } \\
16 . . .\end{array}$ & 35.6 & 13 & 704 & 6.2 & 24.0 & 8.7 & .0 & .0 & 279 & 229 & 2.8 & O. \\
\hline
\end{tabular}

05589735 - PRATRIE OU PONT CREEK NEAR CENTREVILLE. IL ILAT 3831 28 LONG 09006161

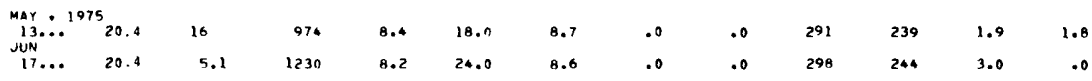

O5594795 - MEAERERS BRANCH NEAR FREEBURG. IL ILAT 382620 LONG OB9 50291

\begin{tabular}{|c|c|c|c|c|c|c|c|c|c|c|c|}
\hline $\begin{array}{l}15 \ldots 15 \\
\operatorname{ltin} \ldots \\
17 \ldots\end{array}$ & $\begin{array}{l}2.55 \\
2.55\end{array}$ & $\begin{array}{c}.89 \\
1.4\end{array}$ & 1790 & $\begin{array}{l}7.9 \\
7.9\end{array}$ & $\begin{array}{l}17.0 \\
22.0\end{array}$ & $\begin{array}{l}8.7 \\
8.3\end{array}$ & $\begin{array}{l}.0 \\
.0\end{array}$ & $\begin{array}{l}.0 \\
.0\end{array}$ & 138 & 113 & $\begin{array}{l}2.8 \\
2.8\end{array}$ \\
\hline
\end{tabular}

05595185 - DOUGLAS CPEEK NEAR SMITHTON. IL (LAT 382528 LONG O89 59 14)

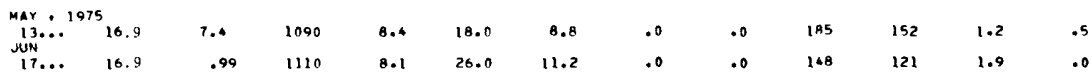

05595190 - WEST FORK RICHLAND CREEK AT FLORAVILLE. IL ILAT 3822 S4 LONG 09002 43)

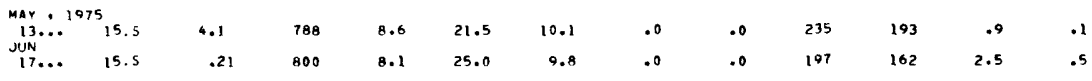

05595230 - DOZA CREEK NEAR NEW ATHENS. IL (LAT 381513 LONG 089 51 (1)

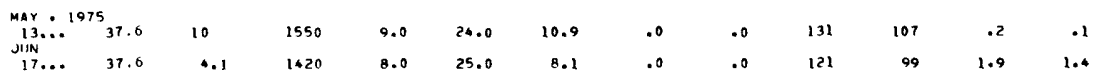

05595295 - LITTLE PLUM CREEK NEAR WALSH. IL ILAT 380713 LONG 0895050 )

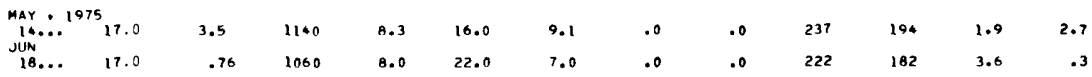


Table 4.--Chemical analyses of samples collected at reconnaissance sites--Continued

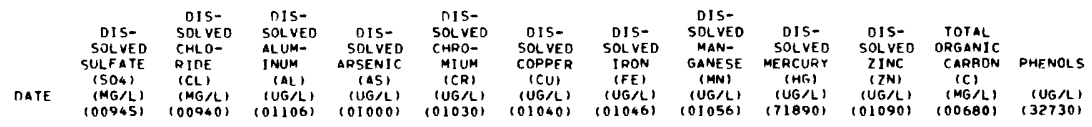

05569920 - COAL CREEK NEAR FAIRYIEW, IL ILAT 403933 LONG 0901400

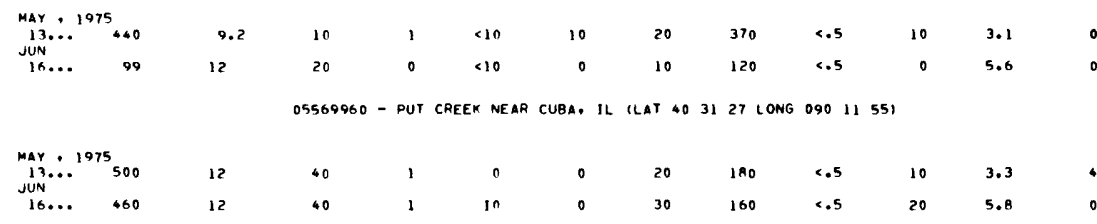

05569970 - TURKEY CREEK NEAR BLYTON, IL (LAT 403326 LONG 09015401

\begin{tabular}{|c|c|c|c|c|c|c|c|c|c|c|c|c|}
\hline I3... & 120 & 14 & 20 & 2 & 10 & 10 & 30 & 100 & $<.5$ & 10 & 6.7 & 34 \\
\hline $16 \ldots$ & 82 & 16 & 20 & 1 & 0 & 0 & 50 & 40 & $<.5$ & 10 & 4.4 & 7 \\
\hline
\end{tabular}

05589735 - PRAIRIE DU PDNT CREEK NEAR CENTREVILLE, IL (LAT 383128 LONG 09006161

\begin{tabular}{|c|c|c|c|c|c|c|c|c|c|c|c|}
\hline${ }_{\text {JUN }} 13 .$. & 270 & 19 & 40 & 2 & $<10$ & 10 & 20 & 530 & .9 & 10 & \\
\hline $17 \ldots$ & 410 & 32 & 40 & 4 & 20 & 10 & 50 & 600 & $<.5$ & 0 & 9.1 \\
\hline
\end{tabular}

05594795 - HEBERERS BRANCH NEAR FREEBURG, IL (LAT 3B 2620 LONG 0895029 )

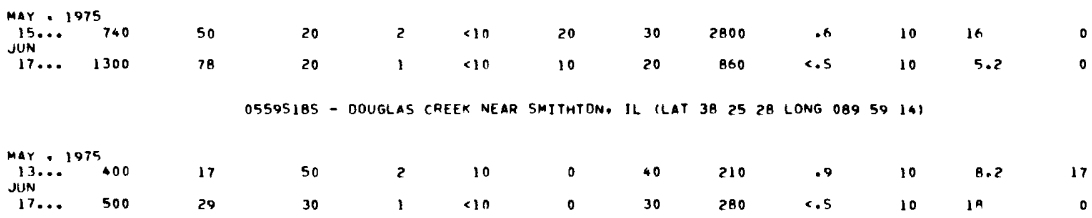

05595190 - WEST FORK RICHLAND CREEK AT FLORAVILLE. IL ILAT 3822 S4 LONG 09002 431

$\operatorname{mar}_{\text {IUN }} 1975190$

15

JiN $17 . . .220$

18

300

$10 \quad 0 \quad 10$

$10 \quad 20 \quad 100$

$100 \quad<.5 \quad 10 \quad 5.6 \quad 0$

OS595230 - DOZA CREEK NEAR NEW ATHENS. IL LLAT 3B 1513 LONG OB9 51411

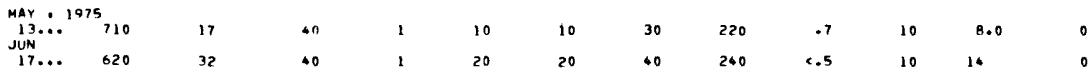

05595295 - L1TTLE PLUM CREEK NEAR WALSH, IL ILAT 3B OT J3 LONG 08950501

\begin{tabular}{|c|c|c|c|c|c|c|c|c|c|c|c|}
\hline JuN & 430 & 13 & 10 & 0 & $<10$ & 20 & 60 & 210 & .6 & 10 & \\
\hline $18 \ldots$ & 380 & 12 & 10 & 1 & 0 & 0 & 20 & 200 & $<.5$ & 0 & 5.2 \\
\hline
\end{tabular}


Table 4,--Chemical analyses of samples collected at reconnaissance sites--Continued

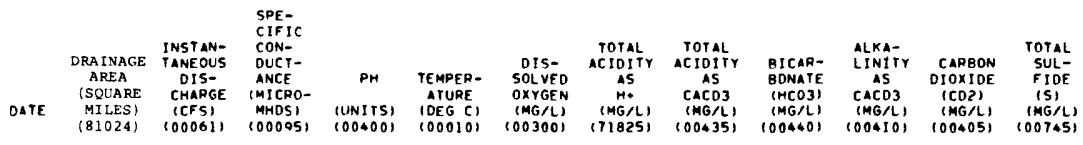

05595505 - Mapys PIVER NEAR STEEleville, IL (LAT 3A 02 OI LONG 089 40 111

\begin{tabular}{|c|c|c|c|c|c|c|c|c|c|c|c|}
\hline JUN $14 .$. & 47.8 & 10 & 1720 & 8.0 & 19.0 & 6.4 & .0 & .0 & 237 & 194 & 3.8 \\
\hline & 47.8 & 1.4 & 1500 & 7.9 & $25 . n$ & 5.8 & .0 & .0 & 221 & 181 & 4.5 \\
\hline
\end{tabular}

OS595520 - NORTH FORK COX CPEEK AT STEELEVILLE. IL ILAT 38 OO 41 LONG O89 38461

\begin{tabular}{|c|c|c|c|c|c|c|c|c|c|c|c|c|}
\hline JUN & 12.8 & 8.5 & 2340 & 8.2 & 20.0 & 7.6 & .0 & .0 & 305 & 250 & 3.1 & .5 \\
\hline $18 \ldots$ & 12.8 & 3.7 & 3180 & 8.3 & 27.0 & 8.4 & .0 & .0 & 305 & 250 & 2.4 & .5 \\
\hline
\end{tabular}

05995530 - WELGE CREEK NEAR STEELEVILLE, IL ILAT 38 OI 33 LONG 08945531

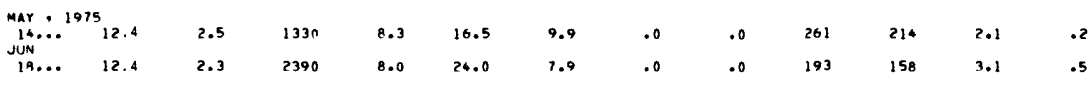

05596110 - ANOY CREEK NEAR CMRISTOPHEA, IL ILAT 375753 LONG 0 OB9 01371

\begin{tabular}{|c|c|c|c|c|c|c|c|c|c|c|c|c|}
\hline IS... & 18.4 & 4.0 & 830 & 7,2 & 17.5 & 6.8 & .0 & .0 & 88 & 12 & 8.9 & .5 \\
\hline IA... & 18.4 & 1.0 & 714 & 0.8 & 22.5 & 4.0 & .0 & .0 & 126 & 103 & 32 & .8 \\
\hline
\end{tabular}

05597035 - PRAIRIE CPEEK NEAP 7EIGLER. IL ILAT 375143 LONG 0890403 I

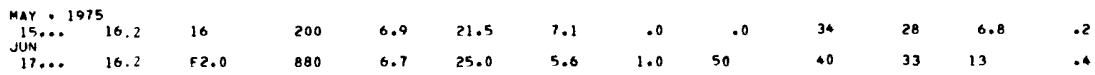

05597045 - LAKE CREEK NEAR HERRIN. IL ILAT 375023 LONG 08900031

\begin{tabular}{|c|c|c|c|c|c|c|c|c|c|c|c|c|}
\hline JUN & 34.0 & $1^{A}$ & 1080 & 6.9 & $21 . n$ & 9.1 & . & .0 & 63 & 52 & 13 & - \\
\hline $17 \ldots$ & 34.0 & 0.0 & -- & 7.1 & 25.1 & 8.8 & .0 & .0 & 57 & 47 & 1.2 & .0 \\
\hline
\end{tabular}

05597050 - PONO CREEK NEAR HERRIN. IL ILAT $3751 \quad 10$ LONG 0890037 )

\begin{tabular}{|c|c|c|c|c|c|c|c|c|c|c|c|c|}
\hline $\operatorname{MaY}_{14 . .}$ & 95.5 & E2? & 1260 & 6.9 & 18.0 & 7.9 & .0 & .0 & 63 & 52 & 13 & 2.3 \\
\hline $17 \ldots$ & 95.5 & 11 & 1980 & 6.7 & 23.5 & 7.1 & .0 & .0 & 57 & 47 & 18 & .0 \\
\hline
\end{tabular}

05597080 - HUPRICANE CREEK NEAP MERRIN. IL (LAT 374916 LONG 0890546 )

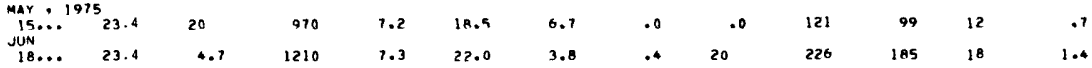


Table 4.--Chemical analyses of samples collected at reconnaissance sites--Continued

\begin{tabular}{|c|c|c|c|c|c|c|c|c|c|c|c|c|}
\hline & $\begin{array}{l}\text { OIS- } \\
\text { SOL VEO } \\
\text { SULFATE } \\
\text { (SOA) }\end{array}$ & $\begin{array}{l}\text { DIS- } \\
\text { SOL VED } \\
\text { CHLO- } \\
\text { RINE } \\
\text { (CL) }\end{array}$ & $\begin{array}{l}\text { DIS- } \\
\text { SOL VED } \\
\text { ALUM- } \\
\text { INUM } \\
\text { (AL) }\end{array}$ & $\begin{array}{l}\text { OIS- } \\
\text { SOLVED } \\
\text { ARSENIC } \\
\text { (AS) }\end{array}$ & $\begin{array}{l}\text { DIS- } \\
\text { SOLVED } \\
\text { CHRO- } \\
\text { MIUM }\end{array}$ & $\begin{array}{l}\text { DIS- } \\
\text { SOLVED } \\
\text { COPPER } \\
\text { (CU) }\end{array}$ & $\begin{array}{l}\text { OIS- } \\
\text { SOLVEO } \\
\text { TRON }\end{array}$ & $\begin{array}{l}\text { OIS- } \\
\text { SOLVED } \\
\text { MAN- } \\
\text { GANESE }\end{array}$ & $\begin{array}{l}\text { OIS- } \\
\text { SOLVEO } \\
\text { MERCURY }\end{array}$ & $\begin{array}{l}\text { OIS- } \\
\text { SOLVED } \\
\text { ZINC }\end{array}$ & $\begin{array}{l}\text { TOTAL } \\
\text { ORGANIC } \\
\text { CARBON }\end{array}$ & PHENOLS \\
\hline ATE & $\begin{array}{l}(504) \\
(M G / L) \\
(00945)\end{array}$ & $\begin{array}{c}\text { (CL) } \\
(M G / L) \\
(00940)\end{array}$ & $\begin{array}{c}(A L) \\
(U G) \\
(01106)\end{array}$ & $\begin{array}{r}(A S) \\
(U G) \\
(01000)\end{array}$ & $\begin{array}{r}\text { (CD) } \\
(U G / 2) \\
(01030)\end{array}$ & $\begin{array}{r}\text { (CU) } \\
(U G / 2) \\
(01040)\end{array}$ & $\begin{array}{c}(F E) \\
(U G / L) \\
(01046)\end{array}$ & $\begin{array}{c}\text { (MN) } \\
(\text { UNG }) \\
(01056)\end{array}$ & $\begin{array}{c}\text { (HG) } \\
(U G / L) \\
(71 B 90)\end{array}$ & $\begin{array}{r}(7 N) \\
(U G /) \\
(01090)\end{array}$ & $\begin{array}{c}(C) \\
(M G) \\
(00680)\end{array}$ & $\begin{array}{r}(U G / L) \\
(32730)\end{array}$ \\
\hline
\end{tabular}

05595505 - MARYS RIVER NEAR STEFLEVILLE. IL ILAT 380201 LONG 08940111

\begin{tabular}{|c|c|c|c|c|c|c|c|c|c|c|c|}
\hline $\operatorname{MUY}_{14}$ & -- & -- & 20 & 1 & $<10$ & 10 & BO & 1600 & 9.5 & 20 & \\
\hline $18 \ldots$ & $64 n$ & 17 & 20 & 0 & 0 & 0 & 10 & 470 & $<.5$ & 10 & 5.8 \\
\hline
\end{tabular}

05595520 - NORTH FORK COX CREEK AT STEELEVILLE. IL (LAT 380041 LONG 08938 46)

\begin{tabular}{|c|c|c|c|c|c|c|c|c|c|c|c|}
\hline JUN".." & 670 & 18 & 20 & 1 & $2 n$ & 20 & 30 & 1000 & .5 & 20 & 7.5 \\
\hline 1月.. & 1800 & 14 & 20 & 1 & 30 & 20 & 60 & 290 & $<.5$ & 10 & 8.4 \\
\hline
\end{tabular}

0S595530 - delge caeek neap Steeleville, IL ILAT 380133 LONG 08945531

\begin{tabular}{|c|c|c|c|c|c|c|c|c|c|c|c|}
\hline $\begin{array}{l}\text { MaY } \\
14 . .\end{array}$ & 1100 & 11 & 20 & 1 & 10 & 10 & 30 & 180 & $<.5$ & 10 & \\
\hline & 1300 & 6.9 & 10 & 1 & 20 & 20 & 10 & 170 & $<.5$ & 0 & 5.3 \\
\hline
\end{tabular}

05596110 - ANOY CREEK NFAP CHRISTOPHER. IL ILAT 375753 LONG OBO 0137 )

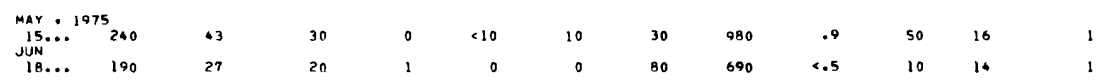

05597035 - PRAIRIE CREEK NEAR TEIGLER, IL ILAT 3751 \$3 LONG OB9 O4 O31

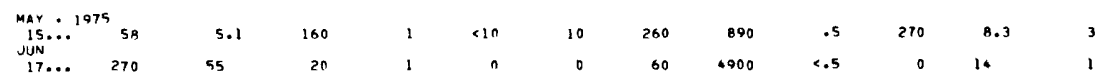

05597045 - LAKE CREEK NEAR HERPIN, IL ILAT 375023 LONG 08900031

\begin{tabular}{|c|c|c|c|c|c|c|c|c|c|c|c|}
\hline JUN & 470 & $1 \mathrm{~A}$ & 60 & 0 & $<10$ & 10 & 100 & 1600 & .6 & 30 & 6.6 \\
\hline $17 \ldots$ & 1100 & 33 & Sn & 0 & 20 & 20 & 10 & 3200 & .5 & 30 & 5.0 \\
\hline
\end{tabular}

05597050 - PONO CREEK NEAR MERRIN, IL ILAT 375110 LONG 08900371

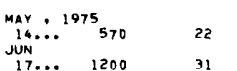

\begin{tabular}{|c|c|c|c|c|c|c|c|c|}
\hline 30 & 1 & 0 & 10 & 70 & 1600 & .9 & 90 & 7.3 \\
\hline 40 & 1 & $<10$ & 10 & 60 & 2600 & .6 & 110 & 17 \\
\hline
\end{tabular}

05597080 - MURAICANE CREEK NEAR HERRIN. IL ILAT 374916 LONG 089 05 46)

\begin{tabular}{|c|c|c|c|c|c|c|c|c|c|c|c|}
\hline $\begin{array}{c}15 \ldots \\
\text { HN } \\
18 . . .\end{array}$ & 360 & 15 & 30 & 1 & 10 & 10 & 110 & 1500 & .7 & 30 & 12 \\
\hline
\end{tabular}


Table 4---Chemical analyses of samples collected at miscellaneous sites--Continued

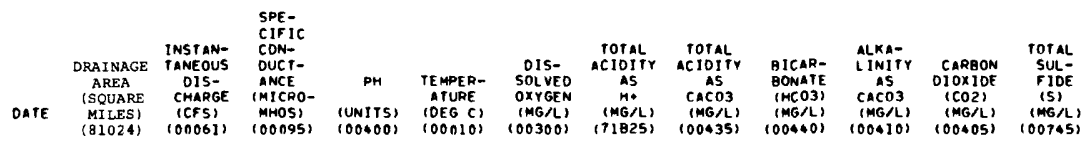

OSS972SS - REESE CREEK NEAR OLO DU OUOIN, IL ILAT 37 SB 33 LONG 089 1002 O

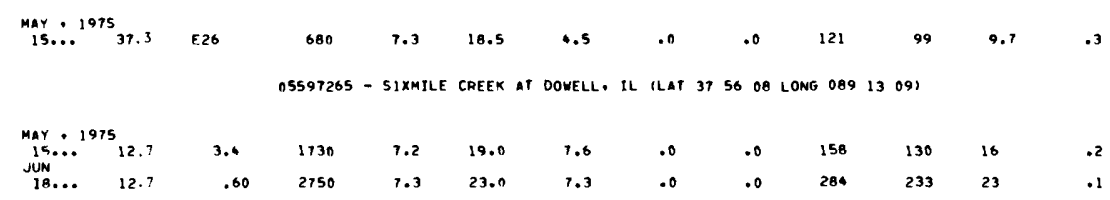

05597490 - LITTLE CRAB ORCHARD CREEK NEAR MARION. IL (LAT 37 44 22 LONG DBB 51211

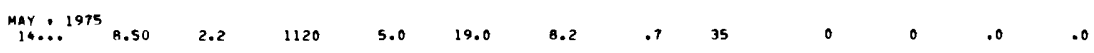

05599020 - GALUM CREEK NEAR LOST PRALRIE, IL ILAT 38 OS 13 LONG O8O 33141

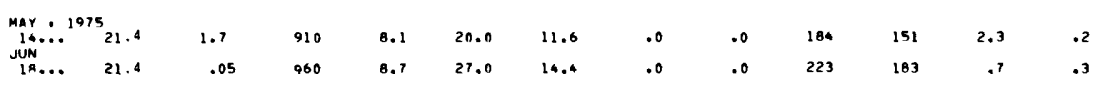

05599055 - PIPESTONE CREEK NEAR DENMARK, IL ILAT 375758 LONG OB9 $2903 \%$

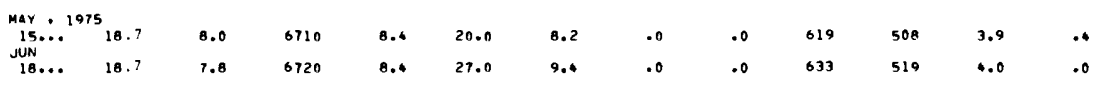
05599060 - BRUSHY FORK NEAR DENMARK. IL ILAT 37 5Y S2 LONG OB9 29 031

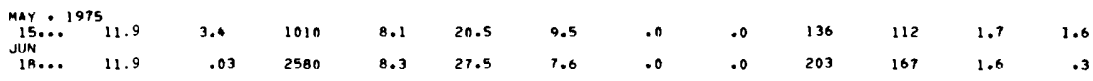


Table 4.--Chemical analyses of samples collected at reconnassance sites--Continued

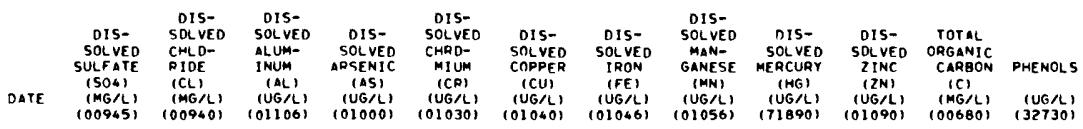

OS597255 - DEESE CREEK NEAR DLD OU OUOIN, IL ILAT 37 SB 33 LONG OB9 1002 I

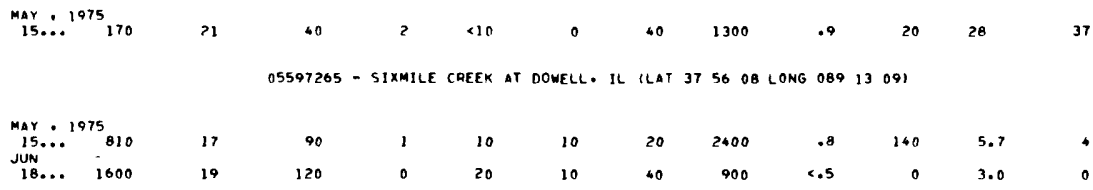

n5597490 - LITTLE CAAB ORCHAPO CREEK NEAR MARION. IL ILAT 37 A4 22 LONG O8B S1 211

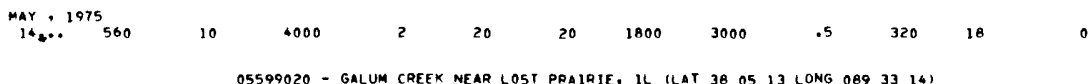

05599020 - GALUM CREE NEAR LOST PRAIRIE. IL ILAT 380513 LONG OB9 33 14)

\begin{tabular}{|c|c|c|c|c|c|c|c|c|c|c|c|}
\hline JUN & 250 & 18 & 20 & 1 & $<10$ & 10 & 60 & 510 & $<.5$ & & \\
\hline $1 A \ldots$ & 250 & 22 & 20 & 1 & $n$ & 0 & 40 & 1300 & $<.5$ & 10 & 8.1 \\
\hline
\end{tabular}

0559905S - PIPESTONE CPEFK NEAR DENMARK, IL ILAT 375758 LONG 08929031

\begin{tabular}{|c|c|c|c|c|c|c|c|c|c|c|c|c|}
\hline${ }_{J U N} 15 . .$. & 3000 & 41 & 20 & 1 & 10 & 10 & 50 & 680 & .6 & 10 & 6.9 & 0 \\
\hline 1в... & 3500 & 45 & 30 & 0 & 20 & 20 & 60 & 140 & $<.5$ & 10 & 18 & - \\
\hline
\end{tabular}

n5599060 - BRUSHY FORK NEAR DENMARK. IL ILAT 375752 LONG 08929031

\begin{tabular}{|c|c|c|c|c|c|c|c|c|c|c|c|c|}
\hline JUN & 360 & 11 & 30 & 1 & 10 & 0 & 0 & 130 & .7 & 10 & 9.4 & 0 \\
\hline lR.. & 1300 & 14 & 20 & 0 & 10 & 20 & 60 & 240 & $<5$ & 10 & 4.8 & $\cdots$ \\
\hline
\end{tabular}


Table $5, \cdots$ Chemical analyses of samples from Saline and Big Muddy River basins

Samples collected and analyzed by the Illinois Environmental Protection Agency are indicated by an asterisk ("). Metal analyses by IEPA are made on acidified water-suspended sediment mixture. The nuaber in parentheses preceding the station number and name refers to the map location on figure 4.

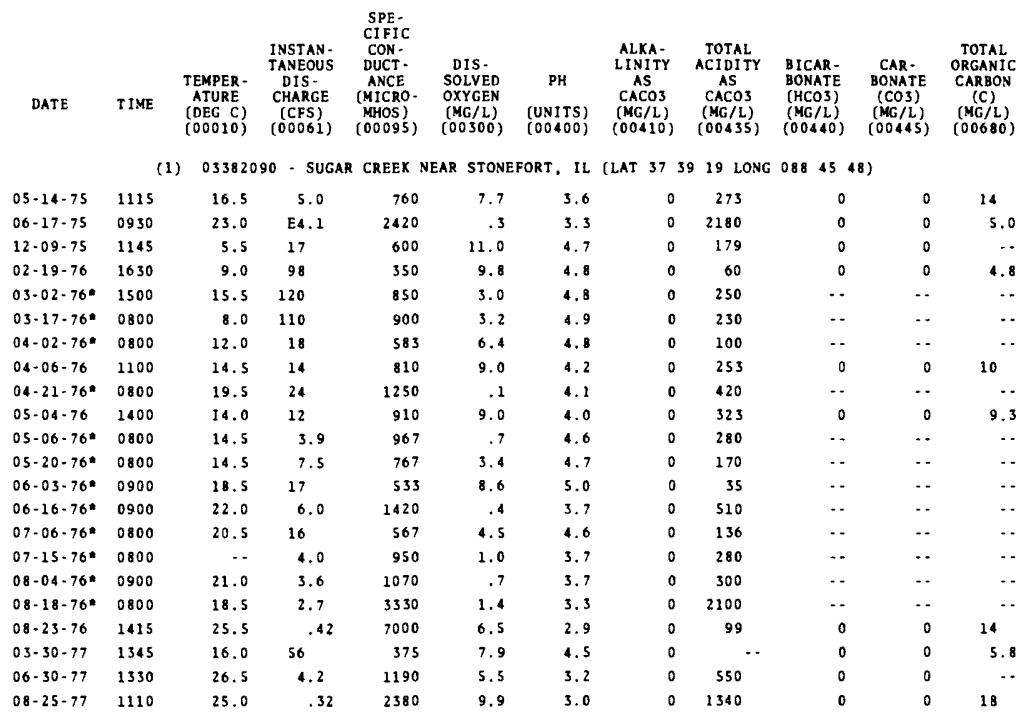

(2) 03382100 - SOUTH FORK SALINE RIVER NEAR CARRIER MILLS, IL (LAT 373816 LONG O88 40 40)

\begin{tabular}{|c|c|c|c|c|c|c|c|c|c|c|c|}
\hline $12 \cdot 09-75$ & 1430 & 5.5 & 126 & 685 & 11.6 & 6.1 & 8 & 15 & 10 & 0 & -. \\
\hline $02-20-76$ & 1030 & 9.0 & 1370 & 260 & 11.0 & 0.2 & 16 & 10 & 20 & 0 & 7.0 \\
\hline $03-04-76^{*}$ & 0900 & 15.5 & 85 & 1070 & 8.6 & 5.9 & 0 & 50 & - & -- & - \\
\hline $03-17-76 *$ & 0900 & 6.5 & 38 & 1170 & 9.8 & 5.1 & 0 & 70 & -. & .. & -. \\
\hline $03-25 \cdot 76$ & 1345 & 14.0 & 118 & $\$ 15$ & 9.8 & 6.2 & 10 & 10 & 12 & 0 & 6.5 \\
\hline $04-02.76^{*}$ & 0900 & 12.0 & 95 & $\$ 50$ & 10.0 & 6.2 & 4 & 0 & $\cdots$ & $\cdots$ & $\cdots$ \\
\hline $04-21-76=$ & 0900 & 19.5 & 42 & 1150 & 8.1 & 3.9 & 0 & 110 & .. & -- & .. \\
\hline $05-06.76 *$ & 0900 & 16.5 & 42 & 1150 & 7.8 & 4.9 & 0 & 60 & $\cdots$ & - & $\cdots$ \\
\hline $05-06-76$ & 1445 & 17.0 & 44 & 1060 & 9.2 & 4.7 & 0 & 65 & 0 & 0 & 6.9 \\
\hline $05-20.76^{\circ}$ & 0900 & 15.5 & 49 & 1100 & 9.1 & 6.4 & 0 & 10 & $\cdots$ & $\cdots$ & $\cdots$ \\
\hline $06-03-76=$ & 0900 & 18.5 & 99 & 583 & 8.6 & 6.4 & 20 & 0 & -. & -. & .. \\
\hline $06-16-76^{*}$ & 0900 & 24.5 & 17 & 1100 & 9.4 & 4.7 & 0 & 140 & $\cdot \cdot$ & $\cdots$ & -. \\
\hline $07-06-76 *$ & 0900 & 22.0 & 796 & 317 & 7.4 & 6.1 & 0 & 0 & $\cdots$ & $\cdots$ & $\cdots$ \\
\hline $07 \cdot 15 \cdot 76^{\circ}$ & $\cdots$ & 26.5 & 26 & 1130 & 7.8 & 4.6 & 0 & so & - & - & - \\
\hline $08-24-76$ & 1530 & 26.0 & 3.1 & 1570 & 7.0 & 3.4 & 0 & 5.0 & 0 & 0 & 12 \\
\hline $10-27-76$ & 1000 & 10.0 & 11 & 1480 & 9.6 & 3.4 & $\cdots$ & 292 & $\cdots$ & $\cdots$ & $\cdots$ \\
\hline $11-18-76$ & 1245 & 7.5 & 4.9 & 2250 & 10.2 & 4.0 & $\cdots$ & 227 & $\cdots$ & $\cdots$ & $\cdots$ \\
\hline $12 \cdot 10 \cdot 76$ & 1100 & 2.5 & 5.5 & 1700 & 13.6 & 3. 8 & $\cdots$ & 458 & $\cdots$ & -. & $\cdots$ \\
\hline $01-12-77$ & 1430 & .0 & 3.8 & 1490 & 7.4 & 3.9 & -. & 412 & $\cdots$ & $\cdots$ & $\cdots$ \\
\hline $02-03-77$ & 1430 & 1.0 & 7.0 & 1360 & -. & 3.5 & $\cdots$ & 306 & 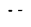 & $\cdots$ & $\cdots$ \\
\hline $03.03-77$ & 1230 & 6.5 & 55 & 1040 & $\cdots$ & 4.9 & $\cdots$ & 64 & $\cdots$ & - & - \\
\hline $03-29-77$ & 1400 & 15.5 & 4710 & 220 & 7.3 & 6.0 & 8 & - & 16 & 0 & 5.4 \\
\hline $05-05.77$ & 1500 & 22.0 & 55 & 1130 & $\cdots$ & 4.0 & $\cdots$ & 90 & $\cdots$ & -. & $\cdots$ \\
\hline 06.09 .77 & 1315 & 24.5 & 4.9 & 3000 & 7.6 & 4.1 & $\cdots$ & 164 & $\cdots$ & $\cdots$ & - \\
\hline 07.01 .77 & 1240 & 26.5 & 24 & 880 & 8.5 & 3.9 & 0 & so & 0 & 0 & 5.4 \\
\hline
\end{tabular}
E Estimated value. 
Table 5.--Chemical analyses of samples from Saline and Big Muddy River basins-Continued

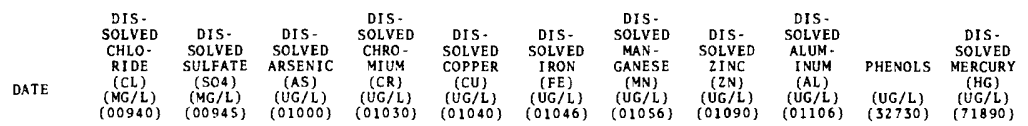

(1) 03382090 - SUGAR CREEK NEAR STONEFORT, IL (LAT 373919 LONG 088 45 48)

\begin{tabular}{|c|c|c|c|c|c|c|c|c|c|c|c|}
\hline$O S-14-7 S$ & 2.1 & 320 & 0 & 70 & 40 & 3700 & $\$ 200$ & $\$ 20$ & 20000 & 27 & 1.7 \\
\hline $06-17-75$ & 6.0 & 2200 & 1 & 600 & 250 & 250000 & 39000 & 3700 & 220000 & 0 & c. 5 \\
\hline $12.09-75$ & 6.4 & 290 & 0 & $<10$ & 10 & 33000 & 4200 & 350 & 14000 & .. & $<.5$ \\
\hline $02-19-76$ & 4.2 & 130 & 0 & $<10$ & 10 & 8000 & 1900 & 140 & 3300 & $\cdots$ & $<. s$ \\
\hline $03-02-76^{\star}$ & 13 & 360 & 0 & 70 & 70 & 44000 & 5500 & 1900 & $\ldots$ & 10 & .4 \\
\hline $03-17-76^{\circ}$ & - & 440 & 0 & 30 & 70 & 46000 & 7000 & 1200 & - & 0 & .7 \\
\hline $04-02-76^{*}$ & -. & 300 & - & $\cdots$ & $\cdots$ & 24000 & 3700 & 500 & $\cdots$ & $\cdots$ & .0 \\
\hline $04-06-76$ & 5.7 & 380 & 0 & 10 & 10 & 44000 & 6000 & 500 & 18000 & .. & $<.5$ \\
\hline $04-21-76 *$ & - & 600 & $\cdots$ & $\cdots$ & -. & 4400 & 11000 & 1900 & $\cdots$ & $\cdots$ & .1 \\
\hline $05.04-76$ & 5.5 & 480 & 0 & 10 & 20 & 48000 & 6500 & 630 & 24000 & .. & $<.5$ \\
\hline $05.06-76 *$ & -. & 440 & $\cdots$ & -. & $\cdots$ & 59000 & 7500 & 1200 & $\cdots$ & 0 & .0 \\
\hline $05-20-76^{\circ}$ & $\cdots$ & 350 & $\cdots$ & . & $\cdots$ & 37500 & 4800 & 700 & - & 0 & .0 \\
\hline $06-03-76 *$ & 8.0 & 140 & 0 & 0 & 0 & 15000 & 2200 & 300 & - & 20 & .0 \\
\hline $06-16-76^{*}$ & 5.0 & 725 & 0 & 20 & 100 & 95000 & 14000 & 2500 & $\cdots$ & 25 & .0 \\
\hline $07-06-76^{\prime \prime}$ & $\cdots$ & 225 & $\cdots$ & $\cdots$ & $\cdots$ & 26500 & -. & - & $\cdots$ & -. & -. \\
\hline $07.15-76^{*}$ & -. & 400 & .. & .. & $\cdots$ & 54000 & -. & .. & - & .. & .. \\
\hline $08-04-76^{*}$ & $\cdots$ & 350 & - & $-\cdot$ & $\cdots$ & 48000 & -- & -. & $\cdots$ & $\cdots$ & $\cdots$ \\
\hline $08-18-76^{\circ}$ & -. & $\cdots$ & . & $\cdots$ & .. & 31500 & .. & $\cdots$ & $\cdots$ & -. & - \\
\hline $08-23-76$ & 6.7 & 5300 & 4 & 100 & 240 & 780000 & 91000 & 8800 & 470000 & -. & $<.5$ \\
\hline $03 \cdot 30-77$ & 4,7 & 160 & 0 & $<10$ & 10 & 9300 & 2200 & 150 & 5400 & .. & c.s \\
\hline $06-30-77$ & 6.5 & 700 & 2 & 15 & 31 & 51000 & 11000 & 1000 & 3700 & .. & .1 \\
\hline $08-25-77$ & 4.4 & 1700 & 0 & 26 & 140 & 100000 & 26000 & 2600 & 140000 & -. & .0 \\
\hline
\end{tabular}

(2) 03382100 - SOUTH FORK SALINE RIVER NLAR CARRIER MILLS, IL (LAT 373816 LONG OB8 40 40)

\begin{tabular}{|c|c|c|c|c|c|c|c|c|c|c|c|}
\hline 12.09 .75 & 6.8 & 320 & 0 & 0 & 0 & 6800 & 3700 & 140 & 390 & -. & $<.5$ \\
\hline $02-20-76$ & 4.6 & 94 & 0 & $<10$ & 0 & 630 & 760 & 60 & 130 & - & $<.5$ \\
\hline $03-04-76^{\circ}$ & .. & 400 & $\cdots$ & $\cdots$ & $\cdots$ & 5500 & 5000 & 400 & -. & 0 & .0 \\
\hline $03.17-76$ & $\cdots$ & 640 & .. & .. & - & 13800 & 6350 & 800 & $\cdots$ & 0 & .7 \\
\hline $03-25-76$ & 6.1 & 230 & 0 & 10 & 0 & 3700 & 2200 & 90 & 30 & -. & $<.5$ \\
\hline $04-02-76 *$ & 8.0 & 220 & 0 & 0 & 0 & 4900 & 2300 & 200 & .. & 0 & .0 \\
\hline $04.21-76^{*}$ & 10 & 680 & 1 & 0 & 50 & $\$ 200$ & 7750 & 700 & - & 5 & .0 \\
\hline $05.06-76 *$ & $-\cdot$ & 560 & - & -. & - & 10000 & 6000 & 400 & $\cdots$ & .. & .4 \\
\hline $0 \$-06-76$ & 5.0 & 190 & 0 & 20 & 20 & 9200 & 5600 & 220 & 3200 & $\cdots$ & $<.5$ \\
\hline $0 S-20-76 *$ & $\cdots$ & 525 & -. & .. & $\cdots$ & 5400 & 4300 & 300 & $\cdots$ & 0 & .0 \\
\hline $06.03-76^{*}$ & .. & 100 & -. & - & -. & 2800 & 1970 & 0 & $\cdots$ & 0 & .0 \\
\hline $06-16-76^{*}$ & . & 550 & .. & -. & . & 1300 & . & -. & - & $\cdots$ & $\cdots$ \\
\hline $07-06-76 *$ & 5.0 & 120 & 0 & 0 & 0 & 2600 & 1100 & 100 & $\cdots$ & 0 & .0 \\
\hline $07.15-76^{*}$ & 8.0 & s50 & 0 & 10 & 50 & 1300 & 4800 & 700 & $\cdots$ & $s$ & .0 \\
\hline $08-24-76$ & 0.6 & 850 & 0 & 10 & 30 & 3200 & 9900 & 770 & 31000 & -. & $<.5$ \\
\hline $10-27-76$ & 13 & 928 & - - & 0 & 20 & 13000 & 10100 & 610 & 13000 & . & -. \\
\hline $11-18-76$ & 10 & 1590 & . & 0 & 20 & 3600 & 13700 & 250 & 3000 & -. & - \\
\hline $12 \cdot 10-76$ & 10 & 1200 & $\cdots$ & 0 & 10 & 28300 & 13300 & 700 & 20000 & $\cdots$ & - \\
\hline $01-12-77$ & 10 & 864 & $\cdots$ & 0 & 20 & 44000 & 10900 & 900 & 30000 & - & $\cdots$ \\
\hline $02-03-77$ & 13 & 783 & -- & 0 & 0 & 30600 & 9360 & 830 & 22000 & 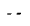 & $\cdots$ \\
\hline $03-03-77$ & 17 & 614 & - & 0 & 0 & 10300 & $\$ 700$ & 280 & 2000 & $\cdots$ & -. \\
\hline 03.29 .77 & 2.0 & 88 & 0 & 0 & 0 & 420 & 780 & 30 & 0 & - & $<.5$ \\
\hline $05.05-77$ & 11 & 672 & .. & 0 & 10 & 2700 & 6800 & 260 & 8000 & $\cdots$ & .. \\
\hline $06-09-77$ & 12 & 2060 & -. & 0 & 10 & 740 & 9800 & 250 & 8000 & $\cdots$ & $\cdots$ \\
\hline $07-01-77$ & 10 & 432 & 2 & 0 & 10 & 630 & 3810 & 230 & 3000 & $\cdots$ & .0 \\
\hline
\end{tabular}


Table 5.--Chemcal analyses of samples from Saline and Big Muddy River basins--Continued

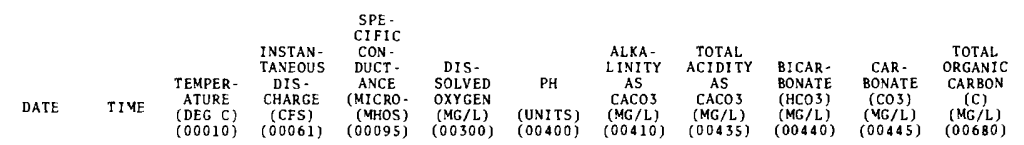

(2) 03382100 - SOUTH FORK SALINE RIVER NEAR CARRIER MILLS, IL (LAT 373816 LONG 08840 40) -. Continued

\begin{tabular}{|c|c|c|c|c|c|c|c|c|c|c|c|}
\hline $08-11-77$ & 1300 & 30.0 & 12 & 2400 & - & 2.9 & - & 1000 & $\cdots$ & $\cdots$ & $\cdots$ \\
\hline $08-29.77$ & 1130 & 26.5 & 6.5 & 1400 & 8.7 & 3.8 & 0 & 84 & 0 & 0 & 8.1 \\
\hline $09-14-77$ & 1345 & 20.0 & 89 & 1140 & 8.8 & 3.1 & - & 300 & - & -. & - \\
\hline
\end{tabular}

(3) 03382130 - SOUTH FORK SALINE RIVER AT MITCHELLSVILLE, IL (LAT $37 \quad 3930$ LONG $08832 \quad 14$ )

\begin{tabular}{|c|c|c|c|c|c|c|c|c|c|c|c|}
\hline $12-12-75$ & 1130 & 8.0 & 113 & 850 & 11.2 & 6.0 & 6 & 25 & 7 & 0 & 3.2 \\
\hline $02-20-76$ & 1520 & 10.0 & 1880 & 278 & 10.0 & 6.5 & 13 & 10 & 16 & 0 & 6.3 \\
\hline $03-04-76^{*}$ & 1000 & 16.0 & 104 & 733 & 8.8 & 5.7 & 0 & 36 & $\cdots$ & $\cdots$ & - \\
\hline $03-17-76^{\prime \prime}$ & 1000 & 6.5 & 67 & 1030 & 10.9 & 5.3 & 0 & 40 & -. & .. & $\cdots$ \\
\hline $03-26-76$ & 1400 & 17.0 & 137 & 560 & 9.3 & 5.8 & 2 & 20 & 2 & 0 & 7.2 \\
\hline $04-02-76^{*}$ & 1000 & 12.0 & 152 & 500 & 10.7 & 6.3 & 0 & 8.0 & -- & .. & .. \\
\hline $04-21-76^{*}$ & 1000 & 18.5 & 65 & 867 & 8.2 & 4.3 & 0 & 60 & .. & -. & .. \\
\hline $05.06 .76^{*}$ & 0900 & 16.5 & 113 & 967 & 8.5 & 4.8 & 0 & 330 & .. & $\ldots$ & $\ldots$ \\
\hline $05-06-76$ & 1430 & 16.0 & 57 & 990 & 9.0 & 4.5 & 0 & 55 & 0 & 0 & 6.8 \\
\hline $05-20-76^{*}$ & 1100 & 16.5 & 80 & 767 & 9.5 & 6.5 & 0 & 4.0 & - - & .. & - \\
\hline $06-16-76^{*}$ & 1100 & 23.5 & 41 & 1130 & 9.0 & 4.0 & 0 & 85 & .. & .. & -. \\
\hline $07-06.76^{\circ}$ & 0900 & 23.0 & 800 & 317 & 8.4 & 6.5 & 6 & 0 & -- & .. & $\cdots$ \\
\hline $07-15-76 *$ & -. & 21.0 & 36 & 1830 & 6.0 & 3.5 & 0 & 230 & - & .. & .. \\
\hline $08.04-76^{*}$ & 1000 & 21.5 & 45 & 1100 & 7.7 & 3.9 & 0 & 75 & $\cdots$ & .. & .. \\
\hline $08-18-76^{*}$ & 1000 & 26.0 & 48 & 1120 & 7.1 & 4.0 & 0 & 100 & .. & -. & $\cdots$ \\
\hline $08-24-76$ & 1230 & 24.0 & 3.7 & 2000 & 7.0 & 3.5 & 0 & 372 & 0 & 0 & 12 \\
\hline $09-03-76 *$ & 1000 & 21.5 & 38 & 2000 & 4.6 & 3.5 & 0 & 330 & $\cdots$ & $-\cdot$ & $\cdots$ \\
\hline $03-31.77$ & 1645 & 15.5 & 4160 & 350 & 7.5 & 6.9 & 11 & .. & 13 & 0 & 8.2 \\
\hline $06-30.77$ & 1100 & 26.5 & 43 & 1430 & 8.9 & 3.2 & 0 & 200 & 0 & 0 & 11 \\
\hline $08-24-77$ & 1400 & 26.0 & 10 & 1630 & 8.3 & 3.4 & 0 & 180 & 0 & 0 & 6.5 \\
\hline
\end{tabular}

(4) 03382170 - BRUSHY CREEK NEAR HARCO, IL (LAT $3746 \quad 30$ LONG 0883908 )

\begin{tabular}{|c|c|c|c|c|c|c|c|c|c|c|c|}
\hline $12-10-75$ & 1030 & 2.0 & 4.0 & 570 & 13.8 & 7.6 & 48 & 30 & 58 & 0 & 3.4 \\
\hline $02-24-76$ & 1130 & 8.0 & 10 & 482 & 12,0 & 6.9 & 44 & 10 & 54 & 0 & 3.3 \\
\hline $03-25-76$ & 1440 & 15.0 & 18 & 560 & 11.2 & 7.8 & 53 & 10 & 65 & 0 & 8.0 \\
\hline $05-04-76$ & 1515 & 16.0 & 13 & 640 & 10.4 & 7.8 & 70 & 15 & 85 & 0 & 4.2 \\
\hline $03-29-77$ & 1130 & 14.5 & 38 & 330 & 8.9 & 6.9 & 29 & - & 35 & 0 & 5.1 \\
\hline $06-29-77$ & 1130 & 23.0 & .20 & 450 & 4.3 & 7.3 & 49 & .. & 60 & 0 & 5.0 \\
\hline
\end{tabular}

(5) 03382185 - BANKSTON FORK NEAR DORRIS HEIGHTS, IL (LAT 3746 O5 LONG 08B 32 25)

\begin{tabular}{|c|c|c|c|c|c|c|c|c|c|c|c|}
\hline $12-10-75$ & 1330 & 3.0 & 39 & 1840 & 12.2 & 7.3 & 94 & 10 & 114 & 0 & 3.6 \\
\hline $02-24-76$ & 1330 & 9.0 & 95 & 1750 & 12.0 & 7.4 & B6 & 15 & 105 & 0 & 7.3 \\
\hline $03.04-76^{\star}$ & 1300 & 15.5 & 19 & .. & 9.6 & 7.7 & 100 & 0 & . & .. & -- \\
\hline $03-17-76^{*}$ & 1300 & 5.5 & 9.0 & . & 12.5 & 7.6 & 120 & 0 & .. & -. & + \\
\hline $03-26-76$ & 1100 & 16.0 & 29 & 2000 & 9.1 & 7.5 & 112 & 10 & 136 & 0 & 12 \\
\hline $04-02-76 *$ & 1300 & 12.5 & 21 & 1950 & 10.8 & 7.3 & 110 & 0 & $\cdots$ & -- & $\cdots$ \\
\hline $04-21-76 *$ & 1300 & 18.0 & 29 & . & 8.3 & 7.4 & 90 & 0 & $\cdots$ & -- & $\cdots$ \\
\hline $05-06-76=$ & 1300 & 17.0 & 2.0 & $\cdots$ & 8.6 & 7.8 & 150 & 0 & $\cdots$ & - & - \\
\hline $05 \cdot 07-76$ & 1300 & 15.0 & 14 & 2350 & 9.2 & 8.1 & 144 & 10 & 176 & 0 & 10 \\
\hline $05-20-76^{*}$ & 1400 & 19.0 & 2.3 & - & 9.5 & 7.7 & 130 & 0 & $\cdots$ & -- & $\cdots$ \\
\hline $06-03-76=$ & 1300 & 18.0 & 2.0 & - & 8.7 & 7.1 & 65 & 0 & - & -- & $\cdots$ \\
\hline $06-16-76 *$ & 1400 & 23.0 & 9.0 & 2500 & 9.0 & 7.3 & 95 & 0 & -. & . & .. \\
\hline $07.06-76^{*}$ & 1200 & 23.0 & 290 & 1400 & 8.0 & 7.1 & 52 & 0 & $\cdots$ & .. & - \\
\hline $07-15-76 *$ & 1400 & 26.0 & 27 & 2700 & 7.4 & 7.5 & 150 & 0 & $\cdots$ & -- & - \\
\hline $08-04-76^{*}$ & 1300 & 23.5 & 57 & 2570 & 8.4 & 7.5 & 110 & 0 & . & . & .. \\
\hline $08-18-76^{*}$ & 1300 & 23.5 & 45 & 2170 & 8.0 & 7.3 & 90 & 0 & $\cdots$ & -. & $\cdots$ \\
\hline
\end{tabular}


Table 5.--Chemlcal analyses of samples from Saline and Big Muddy River basins--Continued

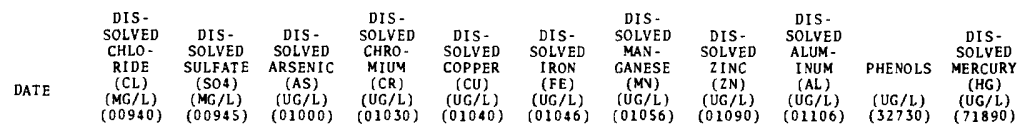

(2) 03382100 - SOUTH FORK SALINE RIVER NEAR CARRIER MILLS, IL (LAT 373816 LONG 08840 40)--Continued

$\begin{array}{rrrrrrrrrrrr}08-11-77 & 8.0 & 1590 & \ldots & 10 & 110 & 46000 & 21000 & 2100 & 92000 & \ldots & . \\ 08-26-77 & 5.3 & 760 & 0 & 2 & 11 & 12000 & 5200 & 240 & 10000 & \ldots & .0 \\ 09-14-77 & 10 & 601 & \ldots & 0 & 30 & 5400 & 7620 & 620 & 25000 & \ldots & \ldots\end{array}$

(3) 03382130 - SOUTH FORK SALINE RIVER AT MITCHELLSVILLE, IL (LAT $37 \quad 3930$ LONG 08832 14)

\begin{tabular}{|c|c|c|c|c|c|c|c|c|c|c|c|}
\hline $12-12-75$ & 7.9 & $\$ 40$ & 0 & 0 & 0 & 0400 & 4900 & 150 & 100 & -. & $<$. \\
\hline $02-20-76$ & 4.5 & 100 & 0 & $<10$ & 10 & 470 & 830 & 30 & 140 & .. & $\ll$ \\
\hline $03-04-76^{*}$ & -. & 180 & . & .. & . & 5000 & 3440 & 300 & -. & -. & \\
\hline $03-17-76 "$ & -. & 552 & - & -. & - & 9800 & 4900 & 400 & -- & $\cdots$ & \\
\hline $03-26-76$ & 7.6 & 240 & 0 & $<10$ & 10 & 2800 & 2600 & 110 & 100 & .. & \\
\hline $04-02-76^{\circ}$ & . & 200 & $\cdots$ & $\therefore$ & . & 3500 & 2250 & 200 & -- & .. & " \\
\hline $04-21-76 n$ & -. & 420 & .. & .. & .. & 5000 & 5800 & 500 & -. & .. & .. \\
\hline $05-06-76 *$ & 8.0 & 450 & 0 & 0 & 0 & 7600 & 4900 & 400 & -. & . & .4 \\
\hline $05-06-76$ & 5.9 & 180 & 0 & 10 & 20 & 4800 & 5300 & 240 & 3100 & - & $<.5$ \\
\hline $05-20-76 *$ & 8.0 & 300 & 0 & 0 & 50 & 3700 & 3000 & 200 & .. & 5 & .8 \\
\hline $06-16-76^{*}$ & .. & 525 & $\cdots$ & -. & - & 6900 & -- & $\cdots$ & -- & .. & .. \\
\hline $07-06-70^{*}$ & -. & 110 & . & -. & .. & 3400 & -. & -. & .. & -. & -. \\
\hline $07-15-76$ & 10 & 880 & 1 & 30 & 40 & 25200 & 12500 & 1200 & $\cdots$ & $s$ & .0 \\
\hline $08-04-76^{*}$ & 5.0 & 450 & 0 & 40 & 0 & 8000 & 6000 & 900 & -. & 7 & .0 \\
\hline $08-18-76 *$ & $-\cdot$ & 525 & -. & $\cdots$ & -. & 5900 & -. & -. & -. & -. & -. \\
\hline $08-24-76$ & 9.8 & 1500 & 0 & 10 & 40 & 25000 & 23000 & 1300 & 47000 & .. & $<.5$ \\
\hline $09-03-76^{\circ}$ & $\cdots$ & 1000 & $\cdots$ & $\cdots$ & -. & 42000 & + & . & -- & -- & - \\
\hline $03-31-77$ & 5.9 & 140 & 0 & $<10$ & 0 & 170 & 910 & 30 & 110 & -. & $<.5$ \\
\hline $06-30-77$ & 7.3 & 760 & 2 & 6 & 23 & 7900 & 9400 & 710 & 5400 & -. & .0 \\
\hline $08-24-77$ & 6.1 & 880 & 0 & 2 & 10 & 18000 & 10000 & $\$ 60$ & 11000 & - & .0 \\
\hline
\end{tabular}

(4) 03382170 - BRUSHY CREEK NEAR HARCO, IL (LAT $3746 \quad 30$ LONG 0883908 )

$\begin{array}{rcccccccrrrr}12-10-75 & 11 & 210 & 0 & <10 & 0 & 80 & 450 & 10 & 70 & \ldots & <.5 \\ 02-24-76 & 8.7 & 170 & 1 & <10 & 0 & 60 & 360 & 0 & 50 & \ldots & <.5 \\ 03-25-76 & 10 & 220 & 0 & <10 & 0 & 60 & 410 & 10 & 30 & \ldots & <.5 \\ 05-04-76 & 9.6 & 220 & 0 & <10 & 0 & 30 & 330 & 10 & 70 & \ldots & <.5 \\ 03-29-77 & 6.4 & 100 & 0 & <10 & 10 & 230 & 200 & 50 & 160 & \ldots & <.5 \\ 06-29-77 & 9.9 & 130 & 3 & 3 & 6 & 210 & 500 & 20 & 210 & \ldots & .3\end{array}$

(5) 03382185 - BANKSTON FORK NEAR DORRIS HEIGHTS, IL (LAT $3746 \quad 05$ LONG $08832 \quad 25$ )

\begin{tabular}{|c|c|c|c|c|c|c|c|c|c|c|c|}
\hline $12-10-75$ & 21 & 960 & 0 & 0 & 0 & 1900 & 1800 & 140 & 30 & $-\cdot$ & $<.5$ \\
\hline $02-24-76$ & 18 & 870 & 1 & 10 & 0 & 440 & 1800 & 60 & 60 & -. & $<.5$ \\
\hline $03-04-76^{*}$ & 35 & 600 & 0 & 0 & 10 & 1300 & 1470 & 200 & -. & 10 & .0 \\
\hline $03-17-76^{*}$ & 25 & 1500 & 0 & 40 & 50 & 1200 & 1700 & 200 & -. & 0 & .0 \\
\hline $03-26-76$ & 23 & 930 & 0 & $<10$ & 0 & 110 & 1500 & 50 & 60 & - & $<.5$ \\
\hline $04-02-70=$ & - & 1150 & -- & $-\cdot$ & .. & 1300 & 12000 & 200 & -. & .. & .0 \\
\hline $04-21-76^{*}$ & $\cdots$ & 1380 & - & $\cdots$ & - & 1800 & 1550 & 200 & $\cdots$ & $\cdots$ & .2 \\
\hline $05-06-76=$ & $\cdots$ & 1350 & -- & -. & -. & 1100 & 870 & 100 & -. & -. & .0 \\
\hline $05-07 \cdot 76$ & 25 & 1300 & 0 & 10 & 0 & 40 & 1400 & 40 & 40 & . & $<.5$ \\
\hline $05-20-76 *$ & $\cdots$ & 1200 & -- & $\cdots$ & - & 1000 & 1100 & 100 & -- & . & .0 \\
\hline $06-03-76^{*}$ & 30 & 1000 & 0 & 0 & 30 & 3500 & 1800 & 400 & $-\cdot$ & -- & .0 \\
\hline $06-16-76^{\circ}$ & 30 & 1000 & 1 & 0 & so & 2900 & 1400 & 200 & -. & $\cdots$ & .0 \\
\hline $07.06 .76^{\prime \prime}$ & -. & 700 & $\cdots$ & $\cdots$ & -. & 2900 & - & $\cdots$ & -. & $\cdots$ & -. \\
\hline $07-15-76 *$ & .. & 1700 & .. & -. & - & 600 & -. & -. & -- & -. & $-\cdot$ \\
\hline $08-04-76 *$ & -- & 1300 & $\cdots$ & $\cdots$ & -. & 800 & $-\cdots$ & $\cdots$ & $\cdots$ & $\cdots$ & $\cdots$ \\
\hline $08-18-76^{*}$ & -. & -- & .. & $\cdots$ & .. & 1800 & $\cdots$ & - & -. & $\cdots$ & - \\
\hline
\end{tabular}


Table 5.--Chenical analyses of samples from Saline and Big Muddy River basins--Continued

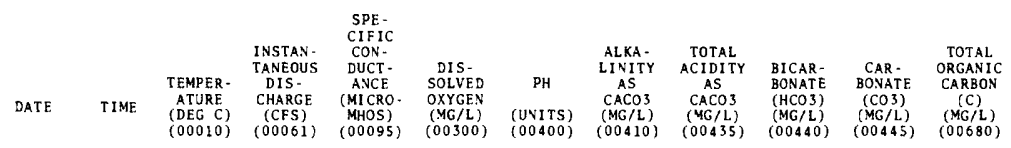

(5) 03382185 - BANKSTON FORK NEAR DORRIS HEIGHTS, IL (LAT 3746 OS LONG 08832 25)-CCONT 1 nued

$\begin{array}{llllrrrrrrrr}08-24-76 & 1030 & 24.0 & 7.7 & 2300 & 7.2 & 7.5 & 127 & 397 & 155 & 0 & 9.0 \\ 09-03-76^{*} & 1300 & 22.0 & .90 & 2170 & 8.0 & 8.6 & 160 & 0 & -. & \ldots & \ldots \\ 03-30-77 & 1600 & 19.0 & 658 & 920 & 7.2 & 6.8 & 36 & . . & 44 & 0 & 8.9 \\ 06-29-77 & 1330 & 25.5 & 23 & 2200 & 8.3 & 6.7 & 11 & \ldots & 14 & 0 & 6.3 \\ 08-25-77 & 1440 & 25.0 & 4.6 & 3450 & 10.3 & 7.6 & 120 & \ldots & 150 & 0 & 4.8\end{array}$

(6) 03382205 - MIDDLE FORK SALINE RIVER NEAR PANKEYVILLF, IL (LAT $3742 \quad 28$ LONG 0882931 )

\begin{tabular}{|c|c|c|c|c|c|c|c|c|c|c|c|}
\hline $12-10-75$ & 1530 & 5.0 & 109 & 1220 & 12.2 & 7.8 & 125 & 15 & 152 & 0 & 4.1 \\
\hline $02-25-76$ & 1500 & 9.5 & $\cdots$ & 1080 & 10.2 & 7.6 & 92 & 15 & 112 & 0 & 5.8 \\
\hline $03-04-76$ & 1100 & 16.5 & $\cdots$ & .. & 9.5 & 7.6 & 120 & 0 & .. & .. & .. \\
\hline $03-17-70$ & 1300 & 0.5 & .. & -. & 13.0 & 7.5 & 120 & 0 & $-\cdot$ & -. & .. \\
\hline $03-26-70$ & 1300 & 18.0 & -. & 1600 & 9.2 & 7.8 & 122 & 15 & 149 & 0 & 8.9 \\
\hline $04-02-76^{*}$ & 1300 & 15.5 & -. & 1480 & 10.3 & 7.5 & 100 & 0 & $\cdots$ & $\cdots$ & -. \\
\hline $04-21-76 *$ & 1300 & 18.5 & . & 1380 & 0.0 & 7.3 & 100 & 0 & .. & - - & -. \\
\hline $05.06-70^{\circ}$ & 1200 & 16.5 & -. & .. & 10.7 & 8.0 & 150 & 0 & $-\cdot$ & -. & .. \\
\hline 05.07 .76 & 1545 & 24.0 & 28 & 1550 & 13.8 & 8.0 & 150 & 10 & 173 & 5 & 11 \\
\hline $05-20-70^{*}$ & 1300 & 21.5 & $\cdots$ & 1400 & 8.7 & 7.6 & 170 & 0 & $\cdots$ & $\cdots$ & -. \\
\hline $06-03 \cdot 76^{*}$ & 1200 & 18.0 & -- & 1230 & 8.0 & 7.4 & 120 & 0 & $-\cdot$ & .. & -. \\
\hline $06-16-70^{*}$ & 1400 & 24.0 & $\cdots$ & 1020 & 10.8 & 7.8 & 150 & 0 & -. & .. & -. \\
\hline $07-06-76 *$ & 1100 & 23.5 & .. & 933 & 7.8 & 7.2 & 84 & 0 & - - & - & -- \\
\hline $07-15-76^{*}$ & 1300 & 28.5 & .. & 2270 & 9.7 & 7.6 & 170 & 0 & $\cdots$ & - & -. \\
\hline $08-04-76 *$ & 1300 & 24.5 & -- & 1920 & 7.7 & 7.4 & 110 & 0 & - & .. & -. \\
\hline $08-18 \cdot 76^{*}$ & 1300 & 26.5 & -- & 2000 & 13.0 & 8.0 & 120 & 0 & -- & $-\cdot$ & -. \\
\hline $08-24 \cdot 76$ & 1345 & 35.0 & 9.2 & 1600 & 19.0 & 9.0 & 126 & 0 & 115 & 19 & 14 \\
\hline $09-03.76 *$ & 1300 & 26.5 & .. & 2830 & 21.9 & 7.8 & 150 & 0 & -- & -- & -. \\
\hline
\end{tabular}

(7) 03382250 - SALINE RIVER AT EQUALITY, IL (LAT 374353 LONG 0882027 )

\begin{tabular}{|c|c|c|c|c|c|c|c|c|c|c|c|}
\hline $12-11-75$ & 1400 & 7.0 & 249 & 950 & 11.0 & 7.6 & 56 & 10 & 68 & 0 & 5.7 \\
\hline $02-25-76$ & 1130 & 10.0 & 548 & 362 & 7.0 & 7.1 & 41 & 10 & so & 0 & 9.4 \\
\hline $03-05-76 *$ & 1400 & 14.5 & .. & 983 & 8.9 & 7.2 & 60 & 0 & -. & -. & -. \\
\hline $03-17-76 *$ & 1200 & 9.0 & - & 1230 & 10.7 & 6.9 & 45 & 0 & .. &.- & $\cdots$ \\
\hline $04-02-70 *$ & 1200 & 15.5 & .. & 750 & 10.4 & 6.9 & 40 & 0 & .. & -. & .. \\
\hline $04-07-76$ & 1100 & 16.0 & 153 & 910 & 9.1 & 7.3 & 39 & 10 & 48 & 0 & 11 \\
\hline $04-21-76 *$ & 1200 & 19.0 & - - & 1070 & 7.3 & 7.3 & 55 & 0 & -. & -. & -- \\
\hline $05-06-70^{\circ}$ & 1100 & 16.5 & - & 1070 & 8.4 & 7.2 & 55 & 0 & -. & -. & -- \\
\hline $0.5-07-70$ & 1045 & 15.0 & 93 & 1180 & 8.6 & 7.3 & 53 & 10 & 65 & 0 & 10 \\
\hline $0.5-20.76^{*}$ & 1300 & 18,5 & $-\cdot$ & 900 & 8.9 & 7.3 & 65 & 0 & -. & $\cdots$ & $\cdots$ \\
\hline $06-03-76 *$ & 1000 & 18.5 & .. & 650 & 8.2 & 7.0 & so & 0 & -. & -. & -. \\
\hline $00-16-76^{*}$ & 1300 & 24.5 & - & 1320 & 10.3 & 7.5 & 65 & 0 & -. & -. & $\cdots$ \\
\hline $0^{4}-06-76^{n}$ & 1100 & 23.5 & .. & 450 & 7.4 & 6.9 & 28 & 0 & -. & -. & -. \\
\hline $07-15-76 n$ & 1200 & 28.0 & $\cdots$ & 1480 & 7.5 & 6.9 & 45 & 0 & .. & $\cdots$ & -- \\
\hline $08-18-76^{\prime \prime}$ & 1200 & 23.5 & $\cdots$ & 1830 & 7.8 & 7.1 & 70 & 0 & -. & $\cdots$ & -. \\
\hline $09-03-76^{*}$ & 1100 & 24.5 & $\cdots$ & 2330 & 12.3 & 8.1 & 90 & 0 & -. & -- & -. \\
\hline
\end{tabular}

(8) 03382320 - RECTOR CREEK NEAR TEXAS CITY, IL (LAT 375301 LONG 08826 05)

\begin{tabular}{|c|c|c|c|c|c|c|c|c|c|c|c|}
\hline $12-11-75$ & 1100 & 4.0 & 18 & 780 & 12.8 & 7.8 & 123 & 10 & 150 & 0 & 4.6 \\
\hline $02-25-76$ & 0945 & 9.0 & 26 & 710 & 12.1 & 7.5 & 121 & 10 & 147 & 0 & 4.4 \\
\hline $03-05.76^{*}$ & 0900 & 13.5 & 145 & 950 & 9.1 & 7.7 & 88 & 0 & -. & -. & -. \\
\hline $03-17-76$ " & 1400 & 7.0 & 0.0 & 907 & 15.4 & 8.0 & 130 & 0 & $\cdots$ & -. & - \\
\hline $04-02-76 n$ & 1400 & 15.0 & 64 & 750 & 12.4 & 7.6 & 95 & 0 & -- & $\cdots$ & - - \\
\hline $04-07-76$ & 1600 & 20.0 & 7.1 & 880 & 13.4 & 8.7 & 139 & 10 & 169 & 7 & 12 \\
\hline
\end{tabular}


Table 5.--Chemical analyses of samples from Saline and Big Muddy River basins--Continued

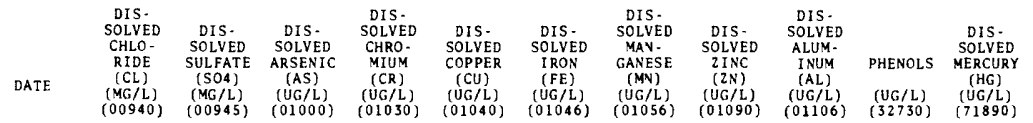

(5) 03382185 - BANKSTON FORX NEAR DORRIS HEIGHTS, IL (LAT 374605 LONG 08832 25)--Contanued

$\begin{array}{lcrrrrrrrrrr}08-24-76 & 13 & 1400 & 0 & 10 & 10 & 60 & 860 & 20 & 970 & \ldots & <.5 \\ 09-03-76^{*} & 15 & 1500 & 0 & 20 & 0 & 700 & 350 & 0 & \cdots & \ldots & .0 \\ 03-30.77 & 9.0 & 430 & 0 & <10 & 0 & 210 & 920 & 40 & 70 & \ldots & <.5 \\ 06-29-77 & 14 & 1300 & 2 & 2 & 0 & 5000 & 2200 & 330 & 60 & \ldots & .0 \\ 08-25-77 & 18 & 2300 & 0 & 1 & 0 & 410 & 1400 & 60 & 200 & \ldots & .0\end{array}$

(6) 03382205 - MIDdLE FORK SALINE RIVER NEAR PANKEYVTLLE, IL (LAT $3742 \quad 28$ LONG 088 29 31)

\begin{tabular}{|c|c|c|c|c|c|c|c|c|c|c|c|}
\hline $12 \cdot 10-75$ & 35 & 490 & 0 & 0 & 0 & 0 & 760 & 20 & 40 & $\cdots$ & $<.5$ \\
\hline $02-25-76$ & 25 & 410 & 1 & $<10$ & 0 & 50 & 860 & 20 & 40 & - - & $<.5$ \\
\hline $03-04-76^{*}$ & - & 750 & -. & - & $\cdots$ & 1000 & 1050 & 100 & $\cdots$ & - & .0 \\
\hline $03-17-76^{\circ}$ & . & 900 & -. & -- & $\cdots$ & 700 & 850 & 100 & - & $\cdots$ & .4 \\
\hline 03.26 .76 & 50 & 610 & 0 & $<10$ & 10 & 30 & 760 & 10 & 30 & . & $<.5$ \\
\hline $04.02 .76^{*}$ & 45 & 600 & 0 & 0 & 50 & 1200 & 520 & 200 & $\cdots$ & 0 & .0 \\
\hline $04-21-76^{k}$ & 105 & 500 & 5 & 0 & 50 & 4400 & 800 & 100 & -. & 5 & .0 \\
\hline $05.06 .70 \mathrm{~A}$ & - & 775 & . & -. & - & 600 & 370 & 0 & - & - & 1 \\
\hline 05.07 .76 & 73 & 720 & 4 & 10 & 0 & 30 & 310 & 10 & 60 & - & $<.5$ \\
\hline $05-20-76^{\star}$ & . & 450 & . & . & - & 1100 & 350 & 100 & .. & $\cdots$ & .0 \\
\hline 06.03 .76 " & $\cdot$ & 400 & $\ldots$ & - & $\cdots$ & 2000 & 600 & 100 & $\ldots$ & . & .0 \\
\hline $06-16 \cdot 76^{\star}$ & -. & 575 & $\cdots$ & -. & $\cdots$ & 1200 & -. & - & $\cdots$ & -. & $\cdots$ \\
\hline $07 \cdot 06 \cdot 76^{*}$ & 20 & 350 & 0 & 0 & 50 & 3600 & 600 & 100 & $\cdots$ & 0 & .0 \\
\hline $07-15-76^{*}$ & 45 & 1200 & 0 & 20 & 50 & 600 & 550 & 0 & - & 10 & .0 \\
\hline $08-04-76^{*}$ & . & 830 & -. & - & $\cdots$ & 600 & .. & - & . & -. & -. \\
\hline $08-18-76^{*}$ & . & 1050 & -. & -- & - & 600 & - & . & - & - & - \\
\hline $08 \cdot 24 \cdot 76$ & 38 & 1100 & 0 & 10 & 10 & 30 & 230 & 10 & 240 & . & $<.5$ \\
\hline $09-03-76^{*}$ & -. & 350 & $\ldots$ & & .. & 300 & .. & . & .. & .. & . \\
\hline
\end{tabular}

(7) 03382250 - SALINE RIVER AT EQUALITY, IL (LAT 374353 LONG 0882027 )

\begin{tabular}{|c|c|c|c|c|c|c|c|c|c|c|c|}
\hline $12-11-75$ & 20 & 410 & 0 & 0 & 0 & 1000 & 2900 & 60 & 30 & . & $<.5$ \\
\hline $02-25-76$ & 14 & 92 & 1 & $<10$ & 0 & 320 & 350 & 0 & 140 & .. & $<.5$ \\
\hline $03-05-76^{*}$ & 20 & 300 & 0 & 20 & 20 & 1600 & 2150 & 100 & .. & 0 & .0 \\
\hline $03-17-76^{*}$ & 33 & 520 & 0 & 20 & 50 & 1300 & 2900 & 100 & .. & 0 & .5 \\
\hline $04-02-76^{*}$ & - & 320 & -. & .. & $\cdots$ & 1300 & 1600 & 100 & $\cdots$ & - & .0 \\
\hline $04-07-76$ & 19 & 380 & 0 & 0 & 0 & 40 & 2000 & 30 & 40 & -. & $<.5$ \\
\hline $04-21-76^{\circ}$ & $\cdots$ & 400 & . & $\cdots$ & -. & 2800 & 2300 & 200 & $\cdots$ & - & . D \\
\hline $05.06-76$ * & $\cdots$ & 540 & - & .. & .. & 2000 & 2550 & 200 & - & - & .0 \\
\hline $05-07-76$ & 23 & 570 & 0 & 10 & 0 & 50 & 3400 & 30 & 40 & $\cdots$ & $<.5$ \\
\hline $05-20-76^{*}$ & - & 350 & -. & .. & .. & 2800 & 1750 & 0 & . & .. & .0 \\
\hline $06-03-76^{\circ}$ & 23 & 150 & 0 & 0 & 30 & 2200 & 1500 & 100 & - & $\cdots$ & .0 \\
\hline $06 \cdot 16-76^{*}$ & 48 & 650 & 0 & 0 & 0 & 1700 & 2700 & 300 & - & $\cdots$ & .0 \\
\hline $07-06-76^{*}$ & - & 150 & -. & -. & $\cdots$ & 3400 & - & -- & - & $\cdots$ & .. \\
\hline $07-15-76^{*}$ & - & 750 & -. & -. & -. & 1400 & -. & - & -. & -. & .. \\
\hline $08-18-76^{*}$ & $\cdots$ & 900 & -. & .. & .. & 1600 & . & -. & - . & .. & -. \\
\hline $09-03-76^{\star}$ & 58 & 1100 & 0 & 20 & 0 & 2100 & 2650 & 0 & -. & 5 & .0 \\
\hline
\end{tabular}

(8) 03382320 - RECTOR CREEK NFAR TEXAS CITY, IL (LAT 375301 LONG 0882605 )

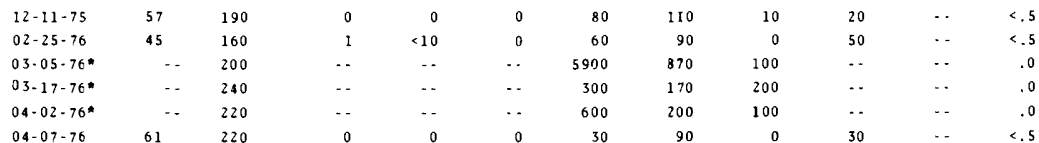


Table 5.--Chemıcal analyses of samples from Saline and Big Muddy River basins--Continued

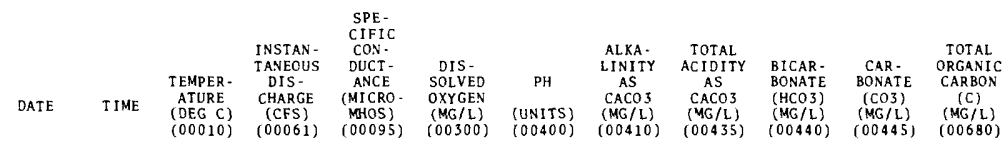

(8) 03382320 - RECTOR CREEK NEAR TEXAS CITY, IL (LAT 375301 LONG 08826 05)--Continued

\begin{tabular}{|c|c|c|c|c|c|c|c|c|c|c|c|}
\hline $04-21-76$ & 1400 & 18.5 & 17 & 750 & 9.0 & 7.8 & 120 & 0 & -- & -- & $\cdots$ \\
\hline $05-06-76$ & 1000 & 17.5 & 3.4 & 920 & 9.8 & 8.0 & 171 & 10 & 208 & 0 & $\cdots$ \\
\hline $05-20-76^{*}$ & 1400 & 23.5 & 0.7 & 817 & 12.8 & 8.0 & 160 & 0 & -. & -- & $\cdots$ \\
\hline $06.03-76$ * & 1300 & 17.5 & 39 & 717 & 9.0 & 7.3 & 110 & 0 & -. & - - & .. \\
\hline $06.16-76^{*}$ & 1500 & 22.5 & 200 & 383 & 6,7 & 1.0 & 0 & 4.0 & -- & -- & .. \\
\hline $07-06-76 *$ & 1300 & 24.5 & 14.5 & 600 & 9.1 & 7.5 & 146 & 0 & - - & -- & $\cdots$ \\
\hline $07-15-76^{*}$ & 1500 & 29.0 & 1.1 & 1080 & 10.3 & 7.9 & 210 & 0 & -. & $-\cdot$ & $\cdots$ \\
\hline $08-04-76^{*}$ & 1400 & 24.5 & .10 & 583 & 9.2 & 7.7 & 280 & 0 & .. & .. & -. \\
\hline $08-18-76^{*}$ & 1400 & 28.0 & .10 & 1070 & 9.2 & 7.9 & 160 & 0 & -. & $\cdots$ & - \\
\hline $08-25-76$ & 1430 & 31.0 & .11 & 1200 & 10.6 & 8.0 & 167 & 0 & 204 & 0 & 14 \\
\hline $09-03-76^{\star}$ & 1400 & 26.0 & .10 & 2670 & $\cdots$ & 7.8 & 130 & 0 & $-\cdot$ & -- & $\cdots$ \\
\hline $03-31 \cdot 77$ & 1230 & 12.5 & 53 & 460 & 8.2 & 7.5 & 90 & -. & 110 & 0 & 6.0 \\
\hline $06-29-77$ & 1500 & 35.0 & 2.3 & 930 & 13.4 & 8.5 & 110 & -. & 130 & 0 & 11 \\
\hline $08-26-77$ & 1520 & 30.0 & 2.9 & 490 & 8.7 & 8.0 & 79 & -. & 96 & 0 & 5.4 \\
\hline
\end{tabular}

(9) 03382450 - SALINE RIVER NEAR EQUALITY, IL (LAT $3742 \quad 14$ LONG $088 \quad 17$ 14)

\begin{tabular}{|c|c|c|c|c|c|c|c|c|c|c|c|}
\hline $12-12-75$ & 1530 & 5.0 & 323 & 920 & 11.6 & 7.5 & 95 & 15 & 110 & 0 & 4.0 \\
\hline $02-25-70$ & 1345 & 10.0 & . & 369 & 8.2 & 7.3 & 54 & 10 & 66 & 0 & 11 \\
\hline $03-05-76^{*}$ & 1300 & 14.5 & . & 767 & 8.4 & 7.4 & 60 & 0 & $\cdots$ & - - & - \\
\hline $03-17-70^{*}$ & 1100 & 10.0 & - - & 1170 & 10.5 & 6.7 & 0 & 36 & - & - & -. \\
\hline $04-02-76 *$ & 1100 & 15.5 & - & 650 & 10.3 & 6.9 & 45 & 0 & $\cdots$ & -. & .. \\
\hline $04-07-76$ & 1300 & 16.0 & $-\cdot$ & 860 & 9.8 & 7.6 & 67 & 10 & 82 & 0 & 16 \\
\hline $04-21-70^{\star}$ & 1100 & 20.0 & - & 1270 & 6.9 & 7.5 & 110 & 0 & $\cdots$ & $\cdots$ & . \\
\hline $05-06-70^{n}$ & 1000 & 18.5 & - - & 1270 & 9.5 & 7.1 & 70 & 0 & -. & - & - \\
\hline $05-06-76$ & 1130 & 18.0 & 116 & 1200 & 9.4 & 7.7 & 74 & 25 & 90 & 0 & 9.7 \\
\hline $05-20-76^{n}$ & 1200 & 18.5 & . & 850 & 8.6 & 7.3 & 120 & 0 & - & - & - \\
\hline $06-03-76$ & 1100 & 19.0 & $\cdots$ & $56 ?$ & 7.9 & 7.1 & 55 & 0 & - & - & -. \\
\hline $06-16-76^{\star}$ & 1200 & 26.0 & $\cdots$ & 1030 & 9.0 & 7.4 & 85 & 0 & - & - & -. \\
\hline $07-06.76 *$ & 1000 & 23.5 & -. & 450 & 6.8 & 7.0 & 56 & 0 & -. & -. & - \\
\hline $07-15-76^{\circ}$ & 1100 & 30.0 & - & 1250 & 8.6 & 7.0 & 70 & 0 & $\cdots$ & $\cdots$ & .. \\
\hline $08-04-70^{n}$ & 1100 & 26.0 & - & 1100 & 6.7 & 6.9 & 55 & 0 & $\cdots$ & - - & . \\
\hline $08-18-70^{n}$ & 1100 & 25.5 & -. & 1480 & 8.5 & 6.9 & 32 & 0 & - - & $\cdots$ & - - \\
\hline $08-25-76$ & 1245 & 27.0 & 17 & 2030 & 8.3 & 7.5 & 103 & 248 & 125 & 0 & 12 \\
\hline $09-03.76^{*}$ & 1200 & 25.0 & . & 1450 & 12.4 & 8.0 & 90 & 0 & - - & .. & -- \\
\hline
\end{tabular}

(11) 05597040 - POND CREEK AT WEST FRANKFORT, IL (LAT 375306 LONG 08855 54)

\begin{tabular}{|c|c|c|c|c|c|c|c|c|c|c|c|}
\hline $12-08-75$ & 1430 & 5.5 & 12 & 520 & 11.0 & 7.7 & 31 & 10 & 38 & 0 & 8.1 \\
\hline $02-19-76$ & 1100 & 9.0 & 60 & 415 & 9.2 & 6.6 & 26 & 10 & 32 & 0 & 17 \\
\hline $03-25-76$ & 1030 & 13.0 & 8,2 & 750 & 10.0 & 7.1 & 59 & 10 & 72 & 0 & 9.7 \\
\hline $05-04-76$ & 1140 & 11.0 & 1.2 & 970 & 10.0 & 7.3 & 61 & 15 & 74 & 0 & 5.6 \\
\hline $08-23-76$ & 1030 & 22.0 & $.0 ?$ & 550 & 5.5 & 7.0 & 51 & 1090 & 62 & 0 & 12 \\
\hline $03-30-77$ & 1100 & 16.0 & 45 & 295 & 6.5 & 8.1 & 13 & . & 16 & 0 & 9.6 \\
\hline $07-01-77$ & 1000 & 22.5 & 5.2 & 520 & 5.6 & 7.2 & 80 & $\cdots$ & 97 & 0 & 5.3 \\
\hline $08-30-77$ & 1115 & 23.0 & 71 & 180 & 7.6 & 6.4 & 30 & .. & 37 & 0 & 6.7 \\
\hline
\end{tabular}

(12) 05597300 - LITTLE MUDDY RIVER NEAR DE SOTn, IL (LAT 374953 LONG 089 11 12)

\begin{tabular}{|c|c|c|c|c|c|c|c|c|c|c|c|}
\hline $01 \cdot 07 \cdot 76$ & 1300 & .0 & 428 & 500 & -- & 7.4 & 43 & 15 & 52 & 0 & - \\
\hline $02-27-76$ & 1430 & 12.0 & 271 & 530 & 9.4 & 7.2 & 55 & 10 & 67 & 0 & 15 \\
\hline $03-31-76$ & 1330 & 15.5 & 357 & 560 & 8.0 & 7.4 & 64 & 10 & 78 & 0 & 15 \\
\hline $05-19-76^{\circ}$ & 1500 & 18.5 & -- & -. & 9.3 & 7.9 & 180 & 0 & -. & -- & \\
\hline $06-02-76 *$ & 1200 & 20.0 & 195 & 600 & 6.6 & 7.3 & 170 & 0 & - & -. & \\
\hline
\end{tabular}


Table i.--Chemical analyses of samples from Saline and Big Muddy River basins-.-Continued

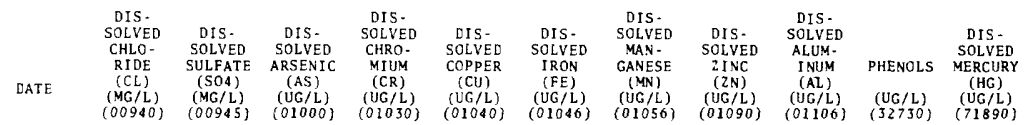

(8) 03382320 - RECTOR CREEK NEAR TEXAS CITY, IL (LAT 375301 LONG 0882605 )--Cont inued

\begin{tabular}{|c|c|c|c|c|c|c|c|c|c|c|c|}
\hline $04-21-76^{*}$ & $\cdots$ & 232 & -- & -. & $\cdots$ & 1800 & 220 & 100 & -. & -. & .7 \\
\hline $05-06-76$ & 62 & 220 & 0 & $<10$ & 0 & 20 & 40 & 0 & 20 & . & $<.5$ \\
\hline $05-20-76^{*}$ & 55 & 190 & 1 & 0 & 50 & 800 & 100 & 0 & - & - & .0 \\
\hline $06.03-76^{*}$ & $\cdots$ & 150 & -. & $\cdots$ & -. & 1300 & 170 & 100 & $-\cdot$ & . & .1 \\
\hline $00-16-76$ & $-\cdot$ & 75 & .. & -- & -- & 25000 & -- & - - & $-\cdot$ & -- & -. \\
\hline $07-06-76^{*}$ & -- & 150 & $\cdots$ & - & $\cdots$ & 1500 & $\cdots$ & -. & $\cdots$ & $\cdots$ & - \\
\hline $07-15 \cdot 76$ * & $\cdots$ & 225 & $\cdots$ & $\cdots$ & $\cdots$ & 400 & -. & $\cdots$ & $\cdots$ & - & $\cdots$ \\
\hline $08.04-76^{*}$ & 30 & 95 & 0 & 30 & 0 & 700 & 140 & 100 & $\cdots$ & $\cdots$ & .0 \\
\hline $08-18 \cdot 76^{*}$ & 75 & 245 & 0 & 0 & 20 & 400 & 100 & 100 & -- & 4 & .0 \\
\hline $08-25-76$ & 87 & 330 & 1 & $<10$ & 10 & 0 & 30 & 10 & 0 & $\cdots$ & $<.5$ \\
\hline $09.03 .76 \mathrm{~m}$ & -0 & & - - & $\cdots$ & $\cdots$ & 700 & $\cdots$ & $\cdots$ & $\cdots$ & $\cdots$ & $\cdots$ \\
\hline $03-31-77$ & 32 & 99 & 1 & $<10$ & 0 & 160 & 80 & 20 & 100 & $\cdots$ & $<.5$ \\
\hline $06-29-77$ & 61 & 360 & 2 & 4 & 6 & 30 & 60 & 10 & 80 & $\cdots$ & $-\cdot$ \\
\hline $08-26.77$ & 27 & 06 & 0 & 1 & 7 & 190 & 100 & 30 & 210 & $\cdots$ & .0 \\
\hline
\end{tabular}

(9) 03382450 - SALINE RIVER VEAR EQUALITY, IL (LAT 37 4214 LONG 08817 14)

\begin{tabular}{|c|c|c|c|c|c|c|c|c|c|c|c|}
\hline $12-12-75$ & 49 & 310 & 0 & 0 & 0 & 200 & 1700 & 40 & 50 & -. & c. 5 \\
\hline $02 \cdot 25 \cdot 76$ & 18 & 92 & 1 & $<10$ & 0 & 320 & 160 & 10 & 170 & $-\cdot$ & $<.5$ \\
\hline $03-05-76 *$ & .. & 200 & - - & 50 & - - & 1800 & 1100 & 100 & .- & .. & .0 \\
\hline $03-17-76$ & -- & 440 & . & . & - & 1000 & 2620 & 200 & - & $\cdots$ & .9 \\
\hline $04-02-76 *$ & -. & 220 & . & .. & -. & 2300 & 1100 & 100 & -. & .. & .0 \\
\hline $04-07.76$ & 35 & 300 & 0 & 0 & 0 & 20 & 1300 & 10 & 40 & .. & $<.5$ \\
\hline $04-21-76^{*}$ & - & 440 & . & - & . & 2000 & 1400 & 100 & -. & -. & .0 \\
\hline $05-06-76 *$ & 55 & 480 & 0 & 0 & 40 & 1200 & 2350 & 100 & - & 5 & .0 \\
\hline $05-06-76$ & 50 & 520 & 0 & 10 & 0 & 10 & 2700 & 0 & 40 & $\cdots$ & $<.5$ \\
\hline $05-20-76^{*}$ & 45 & 225 & 0 & 0 & so & 2200 & 920 & 100 & .. & - & .0 \\
\hline $06-03-76^{*}$ & . & 100 & .. & .. & .. & 4800 & 900 & . & .. & -. & .0 \\
\hline $06-16-76 *$ & . & 350 & -. & .. & - & 1500 & - - & - & $\cdots$ & - & $\cdots$ \\
\hline $07.06-76^{*}$ & -. & 175 & . & . & - & 4200 & . & .. & .. & - & -. \\
\hline $07 \cdot 15 \cdot 76^{*}$ & -. & 575 & .. & .. & -. & 700 & - & -. & .. & $\cdots$ & -- \\
\hline $08-04-76^{*}$ & 25 & 400 & 1 & 40 & 0 & 1600 & 1510 & 100 & -. & 9 & .0 \\
\hline $08-18-76^{*}$ & 23 & 710 & 0 & 30 & 0 & 500 & 0 & 200 & -. & 5 & .0 \\
\hline $08.25-70$ & 45 & 1200 & 0 & $<10$ & 10 & 40 & 2200 & 0 & 60 & - & $<.5$ \\
\hline $09-03-76 *$ & $\cdots$ & 1000 & -. & -. & $\cdots$ & 2100 & - - & .. & .- & -. & -- \\
\hline
\end{tabular}

(11) 05597040 - POND CREEK AT WEST FRANKFORT, IL (LAT $37 \quad 5306 \quad$ LONG 08855 54)

\begin{tabular}{|c|c|c|c|c|c|c|c|c|c|c|c|}
\hline $12-08-75$ & 25 & $1>0$ & 1 & 0 & 0 & 240 & 440 & 20 & 120 & $\cdots$ & $<.5$ \\
\hline $02-19-76$ & 19 & 120 & 0 & $<10$ & 0 & 330 & 550 & 20 & 140 & -- & $<.5$ \\
\hline $03-25-76$ & 43 & 250 & 0 & $<10$ & 0 & 120 & 910 & 10 & 40 & $\cdots$ & $<.5$ \\
\hline $05-04-76$ & 58 & 310 & 0 & $<10$ & 0 & 50 & 390 & 20 & 40 & -. & $<.5$ \\
\hline $08-23-76$ & 48 & 130 & 0 & $<10$ & 10 & 150 & 680 & 10 & 60 & - & $<.5$ \\
\hline $03-30 \cdot 77$ & 17 & 83 & 0 & $<10$ & 0 & 350 & 380 & 20 & 270 & $\cdots$ & $<.5$ \\
\hline $07-01-77$ & 32 & 100 & 0 & 1 & 4 & 50 & 390 & 0 & 180 & $\cdots$ & .0 \\
\hline $08-30-77$ & 9.5 & 38 & 0 & 7 & 8 & 300 & 310 & 10 & 230 & -. & .0 \\
\hline
\end{tabular}

(12) 05597300 - LITTLE MUdDY RIVER NEAR DE SOTO, IL (LAT 374953 LONG 08911 12)

\begin{tabular}{|c|c|c|c|c|c|c|c|c|c|c|c|}
\hline $01-07.76$ & 19 & 120 & 1 & $<10$ & 0 & 230 & 210 & 20 & 130 & $-\cdot$ & $<.5$ \\
\hline $02-27-76$ & 26 & 180 & 1 & $<10$ & 10 & 300 & 300 & 10 & 120 & -- & $<.5$ \\
\hline $03-31-76$ & 24 & 180 & 0 & $<10$ & 10 & 100 & 640 & 0 & 90 & $\cdots$ & $<.5$ \\
\hline $0 S-19-76^{*}$ & -. & 625 & .. & . & .. & 1600 & 2050 & 100 & . & - - & .0 \\
\hline $06-02-76 *$ & .. & 200 & - & -. & - & 5900 & 770 & 100 & $\cdots$ & - & .0 \\
\hline
\end{tabular}


Table 5.--Chemical analyses of samples from Saline and Big Muddy River basins-Continued

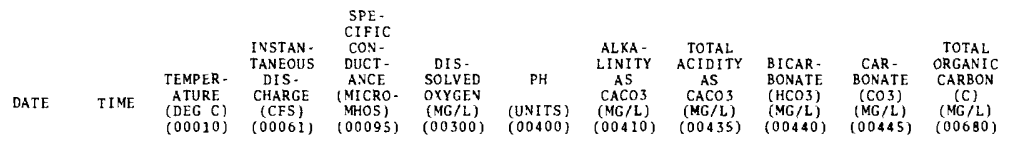

(12) 05597300 - LITTLE MUDDY RIVER NFAR DE SOTO, IL (LAT 374953 LONG 089 11 12)--Continued

$\begin{array}{lllllrlrlrrr}06-15-76^{*} & 1500 & 26.0 & \ldots & 1270 & 0.7 & 7.7 & 105 & 0 & \ldots & \ldots & \ldots \\ 08-17-76^{*} & 1400 & 24.5 & \ldots & 1070 & 11.5 & 7.4 & 100 & 0 & \ldots & \ldots & \ldots \\ 08-30-76 & 1545 & 24.0 & 2.4 & 1570 & 8.5 & 7.7 & 138 & 0 & 168 & 0 & \ldots \\ 04-06-77 & 1445 & 14.0 & 962 & 260 & 5.0 & 6.6 & 36 & \ldots & 44 & 0 & 12 \\ 07-05-77 & 1430 & 29.5 & 24 & 565 & 5.9 & 7.3 & 70 & \ldots & 85 & 0 & 7.9 \\ 08-26-77 & 1415 & 26.0 & 14 & 750 & 11.4 & 8.4 & 77 & \ldots & 94 & 0 & 7.1\end{array}$

(13) 05597500 - CRAB ORCHARD CREEK NEAR MARIOV, IL (LAT $3743 \quad 52$ LONG 0885321 )

\begin{tabular}{|c|c|c|c|c|c|c|c|c|c|c|c|}
\hline $12-09 \cdot 75$ & 0945 & 4.0 & 12 & 910 & 10.8 & $\because 1$ & 26 & 10 & 32 & 0 & 6.4 \\
\hline $02 \cdot 19 \cdot 76$ & 1400 & 10.0 & 69 & 520 & 10.2 & 6.3 & 16 & 15 & 20 & 0 & 9.5 \\
\hline $03-02-76^{*}$ & 1300 & 15.5 & 7.0 & 1330 & 8. 9 & 7.6 & 44 & 0 & -- & .. & . \\
\hline $03-17.76^{*}$ & 0800 & 5.0 & 5.0 & 1380 & 11.4 & 7.5 & 65 & 0 & .. & .. & -. \\
\hline $03-25-76$ & 1230 & 13.0 & 6.7 & 1220 & 9.4 & 7.3 & 67 & 20 & 82 & 0 & 9.1 \\
\hline $04-02-76^{n}$ & 0800 & 10.0 & 8.0 & 1020 & 7.6 & 7.3 & 50 & 0 & .. & .. & -. \\
\hline $04-21-76 *$ & 0800 & 16.5 & 28 & 1050 & 7.7 & 6.2 & 0 & 7.0 & -. & -. & .. \\
\hline $05-06-76^{*}$ & 0800 & 16.0 & -. & 1450 & 7.2 & 7.3 & 80 & 0 & .. & - & .. \\
\hline $05-06-70$ & 1600 & 16.0 & 2.6 & 1420 & 7.0 & 7.3 & 79 & 10 & 96 & 0 & 13 \\
\hline $05-20-76^{k}$ & 0800 & 15.5 & 4.2 & 1130 & 8. 2 & 7.3 & 45 & 0 & $\cdots$ & $\cdots$ & - \\
\hline $06.03 .76 *$ & 0800 & 18.5 & 18 & 733 & 5.3 & 7.0 & 60 & 0 & $\cdots$ & -. & .. \\
\hline $06-16-76^{\star}$ & 0800 & 20.5 & 5.0 & 1080 & 5.8 & 7.1 & 65 & 0 & -. & .. & -. \\
\hline $07-06-76^{*}$ & 0800 & 21.0 & 17 & 767 & 8.0 & 6.7 & 18 & 0 & - & $\cdots$ & - - \\
\hline $07-15-76^{\circ}$ & 0800 & 24.5 & .90 & 1450 & 5.7 & 7.2 & 65 & 0 & -. & $\cdots$ & - \\
\hline $08-23-76$ & 1245 & 24.0 & .34 & 880 & 6.5 & 6.9 & 59 & 0 & 72 & 0 & 18 \\
\hline $03-29-77$ & 1650 & 18.3 & 307 & 342 & 6.4 & 6.1 & 23 & . & 28 & 0 & 8.6 \\
\hline $06-30-77$ & 1500 & 24.5 & .63 & 1180 & 6.2 & 5.0 & 3 & $\cdots$ & 4 & 0 & 6.1 \\
\hline $08-24-77$ & 1145 & 23.5 & .10 & 1140 & 9.3 & 7.3 & 25 & .. & 31 & 0 & 0.9 \\
\hline
\end{tabular}

(14) 05\$98210 - SYCAMORE CREEK NEAR CARBONDALE, IL (LAT 374230 LONG O89 1020 )

\begin{tabular}{|c|c|c|c|c|c|c|c|c|c|c|c|}
\hline $12-24-75$ & 1030 & .0 & 12 & 380 & 13.8 & 7.6 & 95 & 5.0 & 116 & 0 & 2.9 \\
\hline $02 \cdot 27 \cdot 76$ & 1645 & 13.0 & 3.3 & 1500 & 11.7 & 6.1 & 0 & 65 & 0 & 0 & 8.4 \\
\hline $03-31-76$ & 0845 & 11.5 & 7.3 & 1100 & 10.6 & 6.6 & 4 & 35 & 5 & 0 & 6.2 \\
\hline $05-04-76$ & 0830 & 9.0 & 1.1 & 1600 & 11.0 & 4.2 & 0 & 149 & 0 & 0 & 5.6 \\
\hline $08-30-76$ & 1130 & 19.0 & .02 & 3070 & 7.8 & 3.3 & 0 & 94 & 0 & 0 & . \\
\hline $04-07.77$ & 1600 & 17.0 & 4.7 & 1480 & 8.2 & 3.8 & 0 & 240 & 0 & 0 & 3.1 \\
\hline $07-01-77$ & 1440 & 26.0 & 2.6 & 1160 & 8. 4 & 3.5 & 0 & 150 & 0 & 0 & 6.5 \\
\hline $08-29.77$ & 1430 & 23.0 & 6.3 & 570 & 9.8 & 4.9 & 0 & -. & 0 & 0 & 7.2 \\
\hline
\end{tabular}

(15) 05598700 - PANTHER CREEK NFAR PYATTS, IL (LAT 380010 LONG 0892007 )

$\begin{array}{rrrrrrrrrrrr}12-22-75 & 1645 & 2.0 & 6.9 & 3000 & 15.0 & 8.6 & 256 & 20 & 273 & 22 & 3.2 \\ 02-26-76 & 0900 & 9.0 & 14 & 3000 & 11.6 & 7.9 & 195 & 15 & 238 & 0 & 12 \\ 03-30-76 & 1030 & 15.0 & 26 & 1700 & 9.0 & 7.7 & 126 & 30 & 154 & 0 & 14 \\ 05-03-76 & 1100 & 11.5 & 3.5 & 5000 & 9.0 & 8.0 & 240 & 40 & 293 & 0 & \ldots \\ 08-26-76 & 1100 & 26.0 & 2.2 & 4050 & 7.2 & 7.7 & 208 & 10 & 254 & 0 & 9.0 \\ 04-07-77 & 1000 & 12.5 & 10 & 2630 & 9.5 & 7.6 & 200 & \ldots & 240 & 0 & 3.5 \\ 07-07-77 & 1110 & 28.5 & 4.6 & 2880 & 10.4 & 8.0 & 62 & \ldots & 76 & 0 & 4.9 \\ 08-26-77 & 1045 & 24.0 & 4.0 & 3100 & 9.1 & 8.1 & 190 & \ldots & 230 & 0 & 7.4\end{array}$


Table 5.-.Chemical analyses of samples from Saline and Big Muddy River basins--Continued

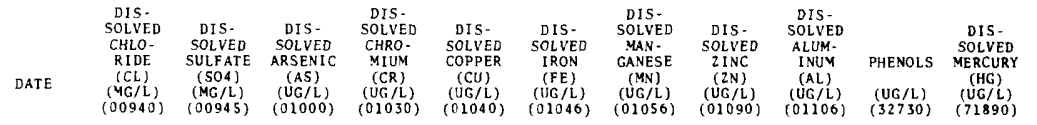

(12) 05597300 - LITTLE MUdDY RIVER NEAR DE SOTO, IL (LAT 374953 LONG 08911 12) - Continued

$\begin{array}{lcrrrrrrrrrr}06-15-76^{*} & \ldots & 540 & \ldots & \ldots & \ldots & 3600 & \ldots & \ldots & \ldots & \ldots & \ldots \\ 08-17-76^{*} & \cdots & 390 & \ldots & \ldots & \ldots & 3100 & \ldots & \ldots & \ldots & \ldots & \ldots \\ 08-30-76 & 20 & 780 & 0 & <10 & 10 & 50 & 13000 & 0 & 20 & \ldots & <.5 \\ 04-06-77 & 8.3 & 80 & 1 & <10 & 0 & 200 & 180 & 30 & 110 & \ldots & <.5 \\ 07-05-77 & 19 & 170 & 2 & 1 & 0 & 100 & 820 & 0 & 220 & \ldots & .0 \\ 08-26-77 & 25 & 230 & 0 & 6 & 0 & 510 & 550 & 0 & 190 & \ldots & .0\end{array}$

(13) 05597500 - CRAB ORCMARd CREEK NEAR MARION, IL (LAT 374352 LONG 08353 21)

\begin{tabular}{|c|c|c|c|c|c|c|c|c|c|c|c|}
\hline $12-09-75$ & 18 & 430 & 0 & 0 & 0 & 1600 & 2300 & 110 & 80 & $\cdots$ & $<.5$ \\
\hline $02 \cdot 19-76$ & 11 & 210 & 0 & $<10$ & 0 & 850 & 1200 & 80 & 140 & $\cdots$ & $<.5$ \\
\hline $03-02-76$ & 38 & 480 & 0 & 70 & 50 & 900 & 2600 & 0 & . & $\ldots$ & .0 \\
\hline $03-17-76^{*}$ & 20 & 500 & 0 & 10 & 50 & 900 & 2000 & 200 & - & $\ldots$ & .0 \\
\hline $03-25-76$ & [ 9 & 530 & 0 & $<10$ & 0 & 90 & 2600 & 30 & 40 & - & $<.5$ \\
\hline $04-02-70^{*}$ & - & 400 & -. & -. & -. & 1400 & 2000 & 200 & -. & $\cdots$ & .0 \\
\hline $04-21-76^{*}$ & $\cdots$ & 552 & $\cdots$ & - & . & 5400 & 3000 & 400 & - & $\cdots$ & .2 \\
\hline $05.06 .76^{*}$ & -- & 752 & -. & $\cdots$ & -. & 1400 & 2100 & 100 & -. & -. & .0 \\
\hline $05-06-76$ & 16 & 79 & 0 & 10 & 0 & 70 & 2500 & 30 & 30 & $\cdots$ & $<.5$ \\
\hline $05-20-76 *$ & 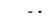 & 475 & -. &.- & - - & 1100 & 1720 & 100 & -. & $\cdots$ & .0 \\
\hline $06-03-76$ " & 10 & 200 & 0 & 0 & 30 & 1300 & 1950 & 0 & $\cdots$ & 20 & .0 \\
\hline $06-16-70^{*}$ & 18 & 425 & 0 & 0 & 50 & 2200 & 1180 & 0 & -. & 15 & .0 \\
\hline $07-06-76 *$ & - & 150 & -. & - & .. & 1500 & .. & . & . & .. & .. \\
\hline $07-15 \cdot 76^{*}$ & $\cdots$ & 750 & $\cdots$ & $\cdots$ & $\cdots$ & 700 & $\cdots$ & $\cdots$ & - & $\cdots$ & $\cdots$ \\
\hline $08-23 \cdot 76$ & 7.6 & 360 & 0 & $<10$ & 10 & 70 & 580 & 10 & 220 & $\cdots$ & $c .5$ \\
\hline $03-29.77$ & 5.5 & 120 & 0 & $<10$ & 10 & 540 & 400 & 40 & 220 & - & $<.5$ \\
\hline $06-30-77$ & 13 & 630 & 2 & 3 & 7 & 340 & 2800 & 230 & 820 & $\cdots$ & .2 \\
\hline $08-24-77$ & 8.4 & 580 & 1 & 1 & 4 & 30 & 1500 & 10 & 20 & .. & .0 \\
\hline
\end{tabular}

(14) 05598210 - SYCAMORE CREEK NFAR CARBONDALE, IL (LAT 374230 LONG 0891020 )

\begin{tabular}{|c|c|c|c|c|c|c|c|c|c|c|c|}
\hline $12 \cdot 24 \cdot 75$ & 11 & 60 & 0 & 0 & 0 & 50 & 660 & 0 & 20 & - & $<.5$ \\
\hline $02-27 \cdot 76$ & 10 & 780 & 1 & $<10$ & 10 & 23000 & 16000 & 390 & 200 & .. & $<.5$ \\
\hline $03-31-76$ & 8.2 & 520 & 0 & $<10$ & 10 & 12000 & 10000 & 200 & 10 & .. & $<.5$ \\
\hline $05-04-76$ & 8.6 & 850 & 0 & 10 & 20 & 17000 & 20000 & 480 & 7800 & $\cdots$ & $<, 5$ \\
\hline $08-30-76$ & 5.7 & 1900 & 0 & 10 & 50 & 9000 & 72000 & 1300 & 49000 & .. & $<.5$ \\
\hline 04.07 .77 & 6.7 & 770 & 0 & $<10$ & so & 20000 & 16000 & 520 & 22000 & - & $<.5$ \\
\hline $07-01-77$ & 5.2 & 600 & 2 & 9 & 26 & 25000 & 15000 & 400 & 2400 & -. & .2 \\
\hline $08-29.77$ & 4.8 & 260 & 1 & 6 & 0 & 1200 & 6500 & 110 & 500 & .. & .0 \\
\hline
\end{tabular}

(15) 05598700 - PANTHER CREEK NEAR PYATTS, IL (LAT 380010 LONG 0892007 )

\begin{tabular}{|c|c|c|c|c|c|c|c|c|c|c|c|}
\hline $12-22-75$ & 18 & [ 500 & 0 & $<10$ & 10 & 50 & 850 & 20 & 20 & $\cdots$ & $<.5$ \\
\hline $02-26-76$ & 17 & 1200 & 1 & 10 & 10 & 49 & 820 & 10 & 30 & -. & $<.5$ \\
\hline $03-30-76$ & 15 & 760 & 0 & $<10$ & 10 & 80 & 750 & 20 & 30 & -- & $<, 5$ \\
\hline 05.03 .76 & 20 & 1900 & 0 & 10 & 10 & 40 & 520 & 10 & 20 & $\cdots$ & $<.5$ \\
\hline $08-26-76$ & 16 & 2440 & 0 & 10 & 20 & 80 & 200 & 10 & 20 & $\cdots$ & $<.5$ \\
\hline $04-07-77$ & 14 & 1700 & 1 & 10 & 10 & 80 & 1100 & 20 & 50 & - & $<.5$ \\
\hline 07.07 .77 & 15 & 1000 & 1 & 0 & 0 & 10 & 200 & 0 & 130 & -. & .0 \\
\hline $08-26-77$ & 17 & 1800 & 0 & 1 & 2 & 30 & 160 & 10 & so & $\cdots$ & .0 \\
\hline
\end{tabular}


Table 5..-Chemical analyses of samples from Saline and Big Muddy Rivet basins--Continued

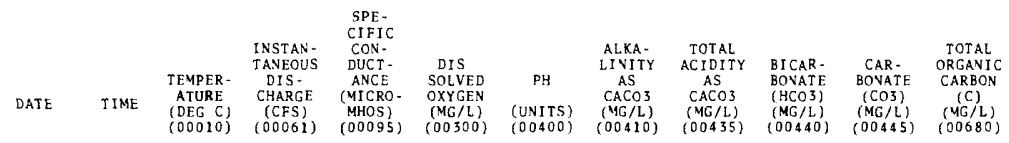

(16) 05599000 - BEAUCOUP CREEK NEAR MATTHEWS, IL (LAT 375800 LONG 0892100 )

\begin{tabular}{|c|c|c|c|c|c|c|c|c|c|c|c|}
\hline $12-23-75$ & $\cdots$ & .0 & 49 & 1450 & 13.6 & 8.0 & 118 & $\cdots$ & 144 & 0 & 8.3 \\
\hline $02-26-76$ & - & 10.0 & 97 & 1040 & 11.2 & 7.6 & 98 & $\cdots$ & 120 & 0 & 11 \\
\hline $03-11-70^{*}$ & 0900 & 9.0 & 83 & 1120 & 9.6 & 7.7 & 230 & 0 & -. & .. & \\
\hline $03-29-76^{*}$ & 1400 & 10.5 & 112 & 1280 & 8.8 & 7.5 & 120 & 0 & - & $\cdots$ & \\
\hline $03-30-76$ & -. & 15.0 & 557 & 860 & 8.0 & 7.5 & 81 & - & 99 & 0 & 24 \\
\hline $04-15-76^{*}$ & 1300 & 17.0 & 18 & 1670 & $9 B$ & 8.0 & 170 & 0 & - & $\cdots$ & $\cdots$ \\
\hline $05-03-76$ & -. & 14.5 & 18 & 2000 & 11.6 & 8.3 & 203 & -. & 248 & 0 & 12 \\
\hline $05-20-76^{*}$ & 0800 & 19.0 & 11 & $\cdots$ & 8.0 & 7.7 & 210 & 0 & $\cdots$ & $\cdots$ & \\
\hline $06-10-76 *$ & 0800 & 22.0 & 41 & 1320 & 6.2 & 7.6 & 120 & 0 & $\cdot \cdot$ & . & 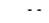 \\
\hline $06-23-76 *$ & 1500 & 22.0 & 10 & $\cdots$ & 8.3 & 7.8 & 180 & 0 & $\cdots$ & $\cdots$ & $\cdots$ \\
\hline $07-15-76^{*}$ & 0800 & 28.0 & 4.2 & 2250 & 5.3 & 7.7 & 170 & 0 & $\cdots$ & $\cdots$ & $\cdots$ \\
\hline $07-29-76^{\prime \prime}$ & 0900 & 23.5 & 193 & 533 & 6.9 & 7.7 & 16 & 0 & $\cdots$ & $\cdots$ & - \\
\hline $08-26-76$ & $\cdots$ & 26.0 & 4.3 & 2720 & 7.6 & 7.8 & 185 & -. & 225 & 0 & 14 \\
\hline $04-07-77$ & 1300 & 13.0 & 120 & 1020 & 8.4 & 7.6 & 90 & - & 110 & 0 & 8.7 \\
\hline $07-07.77$ & 1330 & 29.0 & 10 & 2400 & 5.7 & 7.8 & 7 & -. & $B$ & 0 & 6.3 \\
\hline $08-26.77$ & 1200 & 23.5 & 10 & 1550 & 7.1 & 7.8 & 110 & $\cdots$ & 140 & 0 & 7.2 \\
\hline
\end{tabular}

(17) 05599055 - PIPESTONE CREEK NEAR DENMARK, IL (LAT $37 \quad 5758$ LONG 0892903 )

\begin{tabular}{|c|c|c|c|c|c|c|c|c|c|c|c|}
\hline $05-15-75$ & 1530 & 20.0 & 8.0 & 6710 & 8.2 & 8.4 & 508 & 0 & 619 & 0 & 6.9 \\
\hline $06 \cdot 18-75$ & 1730 & 27.0 & 7.8 & 6720 & 9.4 & 8.4 & 519 & 0 & 633 & 0 & 18 \\
\hline $12-23-75$ & 1600 & .0 & 6.0 & 4680 & 13.2 & 8.6 & 456 & 25 & 517 & 19 & 4.1 \\
\hline $02-27 \cdot 76$ & 0930 & 9.0 & 6.0 & 3700 & 12.0 & 8.3 & 331 & 20 & 404 & 0 & 17 \\
\hline $03-30-76$ & 1600 & 16.0 & 54 & 840 & 9.0 & 7.7 & 100 & 15 & 122 & 0 & \\
\hline $05-03-76$ & 1030 & 14.0 & 3.5 & 3980 & 12.2 & 8.7 & 406 & 30 & 437 & 29 & 8.7 \\
\hline $04-06-77$ & 1215 & 11.0 & 8.2 & 2370 & 11.8 & 8.2 & 190 & - & 230 & 0 & 6.6 \\
\hline $07-06-77$ & 1330 & 35.0 & 1.2 & 2980 & 12.8 & 8.6 & 200 & -. & 230 & 7 & 5.7 \\
\hline $08-25.77$ & 1500 & 27.0 & 4.8 & 5600 & 13.6 & 8.? & 300 & -. & 370 & 0 & 7.5 \\
\hline
\end{tabular}

(18) 05599080 - PIPESTONE CREEK VEAR AVA, IL (LAT $3757 \quad 33$ LONG 0892634 )

$\begin{array}{rrrrrrrrrrrr}12-23-75 & 1430 & .0 & 14 & 2900 & 14.4 & 8.5 & 300 & 15 & 346 & 10 & 4.0 \\ 02-26-76 & 1545 & 13.0 & 13 & 1900 & 12.4 & 8.2 & 207 & 10 & 252 & 0 & 12 \\ 03-30-76 & 1700 & 15.5 & 25 & 1300 & 8.4 & 7.8 & 127 & 20 & 155 & 0 & 18 \\ 05-03-76 & 1715 & 16.5 & .07 & 4360 & 13.0 & 8.6 & 468 & 30 & 517 & 26 & 9.8 \\ 08-27-76 & 1500 & 29.0 & .05 & 6050 & 15.0 & 8.6 & 348 & 20 & 390 & 17 & \ldots \\ 04-06-77 & 1120 & 9.2 & 17 & 1300 & 11.3 & 8.0 & 140 & \ldots & 170 & 0 & 3.6 \\ 07-06-79 & 1500 & 23.5 & 1.0 & 4550 & 12.4 & 8.6 & 230 & \ldots & 260 & 10 & 6.0 \\ 08-25-79 & 1315 & 23.5 & 4.7 & 5300 & 13.0 & 8.7 & 300 & \ldots & 360 & 0 & 6.9\end{array}$

(19) 05599100 - GALUM CREek neAr PYATTS, IL (LAT 375642 LONG 08922 45)

\begin{tabular}{|c|c|c|c|c|c|c|c|c|c|c|c|}
\hline $12 \cdot 23.75$ & 1100 & .0 & 22 & 2020 & 13.4 & 8.3 & 207 & 15 & 252 & 0 & 6.4 \\
\hline $02 \cdot 26-76$ & 1030 & 10.0 & 46 & 1300 & 10.4 & 7.8 & 130 & 10 & 158 & 0 & 12 \\
\hline $03.03 .76^{*}$ & 1000 & 16.5 & 142 & $\cdots$ & 8.6 & 8.1 & 155 & 0 & .. & - & $\cdots$ \\
\hline $03-16-76^{n}$ & 0300 & 9.0 & 34 & . & 13.0 & 8.2 & 190 & 0 & - & $\cdots$ & $\cdots$ \\
\hline $03-30-76$ & 1345 & 15.0 & 556 & 770 & 7.4 & 75 & 80 & 20 & 98 & 0 & 25 \\
\hline $04-01-76 *$ & 1000 & 14.0 & 94 & 2020 & 9.3 & 7.3 & 90 & 0 & .. & $\cdots$ & - \\
\hline $04-20-76^{n}$ & 0800 & 19.0 & 27 & $\cdots$ & 6.1 & 7.8 & 240 & 0 & -. & -. & $\cdots$ \\
\hline 05.03 .76 & 1330 & 13.0 & 11 & 4000 & 10.0 & 8.4 & 320 & 15 & 390 & 0 & 7.1 \\
\hline $05-05-76$ & 1000 & 13.5 & 8.6 & - & 10.2 & 8.1 & 290 & 0 & .. & -. & $\cdots$ \\
\hline $05-19-76^{*}$ & 0800 & 14.5 & 4.6 & $\cdots$ & 6.8 & 8. 0 & 280 & 0 & $\cdots$ & $\cdots$ & $\cdots$ \\
\hline $06-02-76$ * & 1000 & 19.5 & 640 & 467 & 6.3 & 3.0 & 150 & 0 & .. & -. & -. \\
\hline $06-15-76 *$ & 0800 & 23.5 & 6.0 & 2830 & 4.1 & 7.7 & 230 & 0 & -. & .. & -. \\
\hline
\end{tabular}


Table 5.-Chemical analyses of samples from Saline and Big Muddy River basins-C Continued

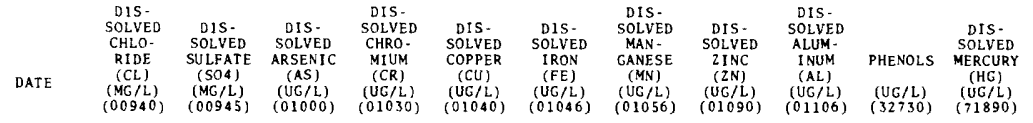

(16) 05599000 - BEAUCOUP CREEK NEAR MATTHEWS, IL (LAT 375800 LONG 0892100 )

\begin{tabular}{|c|c|c|c|c|c|c|c|c|c|c|c|}
\hline $12-23-75$ & 36 & 530 & 0 & 0 & 10 & 40 & 500 & 10 & 40 & $\cdots$ & $<.5$ \\
\hline $02-26-76$ & 31 & 400 & 1 & $<10$ & 0 & 120 & 450 & 0 & 70 & -. & $<.5$ \\
\hline $03-11-76 *$ & $\cdots$ & 400 & .. & -. & $\cdots$ & 2500 & 650 & 0 & -. & -. & .7 \\
\hline $03-29-76^{*}$ & $\cdots$ & 460 &.- & - & $\cdots$ & 1400 & 800 & 0 & $\cdots$ & $\cdots$ & .0 \\
\hline $03-30-76$ & 42 & 270 & 0 & 20 & 10 & 60 & 510 & 0 & 80 & $\cdots$ & $<.5$ \\
\hline $04-15-76^{*}$ & 80 & 940 & 0 & 0 & 30 & 1200 & 1100 & 0 & $\cdots$ & $\cdots$ & .1 \\
\hline $05 \cdot 03 \cdot 76$ & 89 & 850 & 0 & 10 & 0 & 30 & 1000 & 20 & 30 & $\cdots$ & $<.5$ \\
\hline $05-20-76 *$ & -. & 1130 & .. & - & - & 1200 & 1400 & 0 & $\cdots$ & $\cdots$ & .0 \\
\hline $06-10-76 *$ & - & 1000 & $\cdots$ & .. & - & 2400 & 950 & 0 & $\cdots$ & $\cdots$ & .0 \\
\hline $06-23-76$ & $\cdots$ & 1250 & - & . & $\cdots$ & 1100 & 1250 & $\cdots$ & $\cdots$ & - & .1 \\
\hline $07-15-76^{n}$ & 38 & 1400 & 0 & 20 & 70 & 1200 & 1650 & 0 & $\cdots$ & $\cdots$ & .0 \\
\hline $07-29-76^{\circ}$ & 25 & 50 & 1 & 40 & 50 & 6500 & 510 & 0 & $\cdots$ & $\cdots$ & .0 \\
\hline $08-26-76$ & 21 & 1500 & 1 & $<10$ & 10 & 10 & 780 & 10 & 0 & $\cdots$ & $<.5$ \\
\hline $04-07-77$ & 22 & 420 & 1 & $<10$ & 10 & 150 & 490 & 10 & 60 & -. & $<.5$ \\
\hline 07.07 .77 & 16 & 1300 & 2 & 0 & 0 & 10 & 910 & 0 & 130 & $\cdots$ & .0 \\
\hline $08-26 \cdot 77$ & 19 & 700 & 0 & 0 & 3 & 80 & 330 & 0 & 50 & -. & .0 \\
\hline
\end{tabular}

(17) 05599055 - P1PESTONE CREEK NEAR DENMARK, IL (LAT $37 \quad 5758$ LONG 0892903 )

\begin{tabular}{|c|c|c|c|c|c|c|c|c|c|c|c|}
\hline $05-15-75$ & 41 & 3000 & 1 & 10 & 10 & 50 & 680 & 10 & 20 & 0 & .6 \\
\hline $06-18-75$ & 45 & 3500 & 0 & 20 & 20 & 60 & 140 & 10 & 30 & -. & $<.5$ \\
\hline $12-23-75$ & 44 & 2300 & 0 & 10 & 10 & 50 & 900 & 10 & 40 & -. & .6 \\
\hline $02 \cdot 27 \cdot 76$ & 35 & 1700 & 1 & $<10$ & 10 & 60 & 1200 & 10 & 70 & $\cdots$ & 8.5 \\
\hline $03-30-76$ & 12 & 280 & 0 & $<10$ & 10 & 230 & 200 & 0 & 90 & $\cdots$ & $<.5$ \\
\hline $05-03-76$ & 28 & 2000 & 0 & 10 & 10 & 10 & 340 & 0 & 10 & .. & $<.5$ \\
\hline $04-06-77$ & 31 & 920 & 1 & $<10$ & 0 & 60 & 430 & 20 & 80 & $\cdots$ & $<.5$ \\
\hline $07.06 .7^{-1}$ & 70 & 2200 & 0 & 0 & 2 & 10 & 110 & 10 & 200 & $\cdots$ & .0 \\
\hline $08-25 \cdot 77$ & 54 & 2700 & 0 & 0 & 3 & 40 & 1100 & 0 & 60 & .. & 0 \\
\hline
\end{tabular}

(18) 05599080 - PIPESTONE CREEK NEAR AVA, IL (LAT $3757 \quad 33$ LONG 08926 34)

\begin{tabular}{|c|c|c|c|c|c|c|c|c|c|c|c|}
\hline $12 \cdot 23 \cdot 75$ & 30 & 1100 & 0 & $<10$ & 10 & 50 & 600 & 20 & 30 & .. & $<.5$ \\
\hline $02 \cdot 26-76$ & 19 & 710 & 1 & $<10$ & 10 & 60 & 470 & 10 & 40 & .. & $<.5$ \\
\hline $03-30-70$ & 14 & 470 & 0 & $<10$ & 10 & 220 & 350 & 10 & 110 & .. & $<.5$ \\
\hline $05-03.70$ & 32 & 2900 & 1 & 10 & 10 & 40 & 20 & 20 & 10 & .. & $<.5$ \\
\hline $08-27-70$ & 45 & 2300 & 1 & $<10$ & 10 & 30 & 910 & 10 & 20 & $\cdots$ & $<.5$ \\
\hline $04-06-77$ & 18 & 580 & 0 & $<10$ & 0 & 80 & 250 & 10 & 50 & .. & $<.5$ \\
\hline $07-06-77$ & 66 & 2200 & 0 & 0 & 0 & 20 & 190 & 0 & 130 & -. & .0 \\
\hline $08-25-77$ & 51 & 2500 & 0 & 0 & 2 & 50 & 150 & 10 & 40 & .. & .0 \\
\hline
\end{tabular}

(19) 05599100 - GALUM CREEK NEAR PYATTS, IL (LAT 375642 LONG 08922 45)

\begin{tabular}{|c|c|c|c|c|c|c|c|c|c|c|c|}
\hline $12-23-75$ & 26 & 850 & 0 & 10 & 0 & 50 & 520 & 10 & 50 & -. & $<.5$ \\
\hline $02+20-70$ & 21 & 500 & 2 & $<10$ & 0 & 80 & 440 & 0 & 40 & $\cdots$ & $<.5$ \\
\hline $03-03-76 *$ & - & 920 & $\cdots$ & - & $\cdots$ & 1500 & 800 & 100 & $\cdots$ & $\cdots$ & .1 \\
\hline $03-16-76^{*}$ & $\cdots$ & 75 & $\cdots$ & $\cdots$ & $\cdots$ & 800 & 620 & 100 & -. & $\cdots$ & .0 \\
\hline $03-30-76$ & 12 & 290 & 0 & $<10$ & 10 & 170 & 340 & 0 & 140 & -. & $<.5$ \\
\hline $04-01-76^{*}$ & 30 & 200 & 0 & 0 & 50 & 740 & 530 & 100 & $\cdots$ & 15 & .0 \\
\hline $04-20-76$ " & 40 & 1000 & 1 & 0 & 50 & 2300 & 1550 & 300 & $\cdots$ & 10 & .3 \\
\hline $05-03-76$ & 33 & 1400 & 0 & 10 & 10 & 30 & 750 & 10 & 10 & - & $<.5$ \\
\hline $05.05 .76^{*}$ & -. & 1450 & -. & -. & $\cdots$ & 800 & 700 & 100 & -. & $\cdots$ & .4 \\
\hline $05-19-76^{*}$ & $\cdots$ & 3500 & 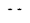 & .. & $\cdots$ & 1000 & 1500 & 100 & $\cdots$ & -. & .0 \\
\hline $06-02-76 *$ & -. & 145 & -. & -. & $\cdots$ & 750 & 700 & 0 & $\cdots$ & - & .0 \\
\hline $06-15-76$ * & -. & 1000 & $\cdots$ & $\cdots$ & $\cdots$ & 2000 & -. & $\cdots$ & . & $\cdots$ & -. \\
\hline
\end{tabular}


Table 5.--Chemical analyses of samples from Saline and Big Muddy River basins--Continued

\begin{tabular}{|c|c|c|c|c|c|c|c|c|c|c|c|c|}
\hline DATE & TIME & $\begin{array}{c}\text { TEMPER- } \\
\text { ATURE } \\
\text { (DEG C) } \\
(00010)\end{array}$ & $\begin{array}{l}\text { INSTAN- } \\
\text { TANEOUS } \\
\text { DIS. } \\
\text { CHARGE } \\
\text { (CFS) } \\
(00061)\end{array}$ & $\begin{array}{l}\text { SPE- } \\
\text { CIFIC } \\
\text { CON- } \\
\text { DUCT- } \\
\text { ANCE } \\
\text { (MICRO- } \\
\text { MHOS) } \\
(00095)\end{array}$ & $\begin{array}{l}\text { DIS- } \\
\text { SOLVED } \\
\text { OXYGEN } \\
\text { (YG/L) } \\
(00300)\end{array}$ & $\begin{array}{c}\text { PH } \\
(\text { UNITS) } \\
(00400)\end{array}$ & $\begin{array}{l}\text { ALK } \\
\text { LIN } \\
\text { AE } \\
\text { CAC } \\
\text { CMG } \\
0004\end{array}$ & $\begin{array}{l}\text { KA- } \\
\text { iITY } \\
\text { is } \\
\text { Co3 } \\
\text { iti } \\
410)\end{array}$ & $\begin{array}{c}\text { TOTAL } \\
\text { ACIDITY } \\
\text { AS } \\
\text { CACO3 } \\
\text { (MG/L) } \\
(00435)\end{array}$ & $\begin{array}{l}\text { BICAR- } \\
\text { BONATE } \\
\text { (HCO3) } \\
(M G / L) \\
(00440)\end{array}$ & $\begin{array}{c}\text { CAR- } \\
\text { BONATE } \\
(\mathrm{CO} 3) \\
(\mathrm{MG} / \mathrm{L}) \\
(00445)\end{array}$ & $\begin{array}{c}\text { TOTAL } \\
\text { ORGANIC } \\
\text { CARBON } \\
(\mathrm{C}) \\
(\mathrm{MG} / \mathrm{L}) \\
(00680)\end{array}$ \\
\hline & (19) & 05599100 & - GALUM & CREEK NEAR & PYATTS, & IL (LAT & 3756 & 42 & LONG 0892 & $2245) \cdots C$ & ntinued & \\
\hline $97-02.76$ & 1000 & 21.5 & 1.0 & 3070 & 5.6 & 7.8 & & 260 & 0 & $\cdots$ & -. & - \\
\hline $08-17.76^{\circ}$ & 0800 & 22.0 & 9.5 & 4350 & 8.6 & 8.0 & & 280 & 0 & $\cdots$ & .. & -. \\
\hline $08-27-76$ & 1200 & 25.0 & 3.1 & 5050 & 8. 3 & 8.1 & & 308 & 238 & 376 & 0 & - - \\
\hline $09-14-76^{*}$ & 0800 & 18.0 & 0.0 & 1120 & 6.2 & 7.6 & & 65 & $\theta$ & $\cdots$ & -. & -. \\
\hline
\end{tabular}

(20) 05599300 - BEAUCOUP CREEX NEAR ORAVILLE, IL (LAT 375143 LONG 0891957 )

$\begin{array}{rrrrrrrrrrrr}12.22 .75 & 1430 & 1.0 & 91 & 1060 & 12.8 & 8.0 & 118 & 20 & 144 & 0 & 7.2 \\ 02-27-76 & 1215 & 11.0 & 172 & 1040 & 8.0 & 7.6 & 113 & 10 & 138 & -. & 12 \\ 03-31-76 & 1045 & 14.5 & 1230 & 760 & 7.8 & 7.3 & 77 & 10 & 94 & 0 & 24 \\ 04-07-77 & 1400 & 13.0 & 403 & 780 & 7.2 & 7.4 & 74 & \ldots & 90 & 0 & 8.4 \\ 07-05-77 & 1300 & 28.0 & 24 & 1380 & 4.7 & 7.5 & 150 & \ldots & 180 & 0 & 6.3 \\ 08.25-77 & 1045 & 23.0 & 41 & 2260 & 8.2 & 8.3 & 160 & \ldots & 190 & 0 & 7.5\end{array}$

(21) *05599500 - BIG MUdDY RIVER AT MURPhYSBORO, IL (LAT 374455 LONG 08920 45)

\begin{tabular}{|c|c|c|c|c|c|c|c|c|c|c|c|}
\hline $11-11-74$ & 1530 & 12.0 & 3290 & 330 & $\cdots$ & 7.1 & 40 & $\cdots$ & 49 & 0 & - \\
\hline $12-10-74$ & 1400 & 3.5 & 1730 & 697 & -. & 6.4 & 77 & -. & 94 & 0 & 10 \\
\hline $01-06-75$ & 1330 & 4.0 & 3510 & 751 & -- & 7.3 & 48 & $-\cdot$ & 58 & 0 & $\cdots$ \\
\hline $02-10-75$ & 1345 & 3.0 & 4450 & 448 & $\cdots$ & 7.0 & 46 & - & 56 & 0 & -- \\
\hline $03 \cdot 11 \cdot 75$ & 1330 & 5.0 & 2850 & 597 & -. & 7.2 & 54 & -. & 66 & 0 & 8.3 \\
\hline $04 \cdot 14-75$ & 1100 & 11.5 & 1500 & 500 & -- & 7.3 & 59 & $\cdots$ & 72 & 0 & $\cdots$ \\
\hline $05-06-75$ & 1130 & 19.5 & 7000 & 360 & $-\cdot$ & 6.8 & 43 & -- & 53 & 0 & - \\
\hline $06.09-75$ & 1200 & 23.0 & 872 & 655 & - & 7.2 & 68 & $\cdots$ & 83 & 0 & $\cdots$ \\
\hline $07.07 \cdot 75$ & 1400 & 25.0 & 1800 & 690 & $-\cdot$ & 6.6 & 54 & $-\cdot$ & 66 & 0 & 7.7 \\
\hline $08 \cdot 18 \cdot 75$ & 1600 & 26.0 & 1040 & 650 & 5.3 & 7.4 & 68 & $-\cdot$ & 83 & 0 & $\cdots$ \\
\hline $09-15-75$ & 1530 & 20.0 & 312 & 805 & 6.5 & 7.3 & 109 & -. & 133 & -- & $\cdots$ \\
\hline $10-15-75$ & 1300 & 20.0 & 89 & 1050 & 9.7 & 7.7 & 121 & $\cdots$ & 147 & 0 & $\cdot-$ \\
\hline $11-10 \cdot 75$ & 1415 & 15.0 & 218 & 1060 & 6.2 & 7.8 & 128 & $\cdots$ & 156 & 0 & $\cdots$ \\
\hline $12-15-75$ & 1630 & 7.0 & 1120 & 355 & 10.0 & 7.5 & 56 & $\cdots$ & 68 & 0 & 15 \\
\hline $12 \cdot 22 \cdot 75$ & 1100 & 1.0 & 1560 & 580 & 12.2 & 7.4 & 51 & 10 & 62 & 0 & 9.4 \\
\hline $01-19-76$ & 1500 & .0 & 683 & 800 & 13.8 & 73 & 84 & - & 102 & 0 & $\cdots$ \\
\hline $02-09-76$ & 1400 & 1.0 & 446 & 930 & 12.3 & 7.3 & 102 & - & 124 & 0 & -. \\
\hline $02 \cdot 27 \cdot 76$ & 0800 & 9.0 & 2500 & 470 & 9.0 & 7.1 & 48 & 10 & 59 & 0 & 10 \\
\hline $03-03-76$ & 1400 & 15.5 & 684 & 733 & 8.7 & 7.6 & 65 & 0 & $-\cdot$ & $-\cdot$ & -- \\
\hline $03-15 \cdot 76$ & 1330 & 11.5 & 702 & 690 & 9.6 & 7.4 & 88 & - & 107 & 0 & 13 \\
\hline $03-16-76^{*}$ & 1100 & 10.0 & 630 & 700 & 10.6 & 7.7 & 75 & 0 & $\cdots$ & $\cdots$ & - \\
\hline $04-01-76^{*}$ & 1500 & 14.5 & 2420 & 583 & 8.0 & 7.2 & 50 & 0 & $\cdots$ & $\cdots$ & $\cdots$ \\
\hline $04-06-76$ & 1515 & 16.0 & 1060 & 610 & 7.6 & 7.3 & 62 & 5.0 & 76 & 0 & 15 \\
\hline $04 \cdot 12 \cdot 76$ & 1345 & 16.0 & 390 & 770 & 9.0 & 7.5 & 89 & -. & 108 & 0 & - \\
\hline $04-20-76^{a}$ & 1100 & 21.0 & 318 & 850 & 7.2 & 7.6 & 100 & 0 & $\cdots$ & $\cdots$ & $\cdots$ \\
\hline $05-05-76^{*}$ & 1400 & 16.5 & 450 & 717 & 11.3 & 7.8 & 68 & 0 & $\cdots$ & -- & $\cdots$ \\
\hline $05-17-76$ & 1400 & 19.0 & 220 & 890 & 5.6 & 7.4 & 110 & - & 134 & 0 & 15 \\
\hline $05-19-76$ & 1100 & 19.0 & 410 & 950 & 7.5 & 7.7 & 110 & 0 & $\cdots$ & $\cdots$ & - \\
\hline $06-02-76^{*}$ & 1400 & 20.0 & 2310 & 633 & 5.2 & 7.3 & 90 & 0 & .. & $\cdots$ & $\cdots$ \\
\hline $06-07.76$ & 1400 & 21.5 & 1860 & 515 & 5.7 & 7.1 & 52 & -. & 63 & 0 & $\cdots$ \\
\hline $06-15-76^{*}$ & 1000 & 26.0 & 288 & 800 & 5.8 & 7.5 & 80 & 0 & $\cdots$ & $\cdots$ & $\cdots$ \\
\hline $07.02 .76^{\circ}$ & 1400 & 24.0 & 514 & 600 & 6.2 & 7.3 & 64 & 0 & $\cdots$ & . & -. \\
\hline $07-12-76$ & 1700 & 28.0 & 609 & 600 & 5.5 & 7.2 & 73 & $\cdots$ & 89 & 0 & -. \\
\hline $07-15-76^{*}$ & 1100 & 30.5 & 487 & 567 & 8.3 & 7,3 & 60 & 0 & $-\cdot$ & - & .. \\
\hline $08.09-76$ & 1530 & 24.5 & 220 & 710 & 6.0 & 7.7 & 83 & -. & 101 & 0 & 8.7 \\
\hline
\end{tabular}

an Discharge values for this station are mean dasly discharges. 
Table 5..-Chemical analyses of samples from Saline and Big Muddy River basins-.Contanued

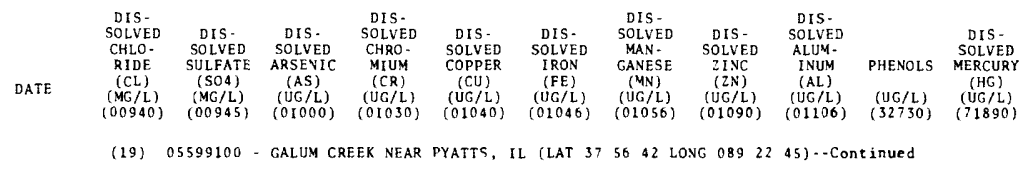

\begin{tabular}{|c|c|c|c|c|c|c|c|c|c|c|c|}
\hline $07-02-76^{*}$ & 40 & 1500 & 1 & 0 & 50 & 2500 & 1000 & 100 & .. & 5 & .0 \\
\hline $08-17-76^{\circ}$ & -. & 2000 & $\ldots$ & - & - & 2100 & - & -. & $\cdots$ & . & -. \\
\hline $08-27-76$ & 52 & 2400 & 1 & $<10$ & 10 & 70 & 580 & 10 & 0 & $\cdots$ & $<.5$ \\
\hline $09-14.76^{*}$ & -. & 500 & $\ldots$ & -. & .. & 1500 & $\ldots$ & .. &.- & $\ldots$ & $\cdots$ \\
\hline
\end{tabular}

(20) 05599300 - BEAUCOUP CREEK VEAR ORAVILLE, IL (LAT 375143 LONG 0891957 )

\begin{tabular}{|c|c|c|c|c|c|c|c|c|c|c|c|}
\hline $12-22-75$ & 32 & 440 & 0 & 0 & 0 & 50 & $5>0$ & 10 & 60 & -. & $<.5$ \\
\hline $02-27 \cdot 76$ & 33 & 430 & 1 & $<10$ & 10 & 100 & 510 & 10 & 50 & $\cdots$ & $<.5$ \\
\hline $03-31-76$ & 39 & 220 & 0 & $<10$ & 10 & 210 & 200 & 10 & 120 & - & $<.5$ \\
\hline $04-07-77$ & 19 & 310 & 1 & $<10$ & 10 & 200 & 550 & 30 & 60 & $\cdots$ & $<.5$ \\
\hline $07-05-77$ & 10 & 1300 & 2 & 0 & 0 & 10 & 910 & 0 & 130 & $\cdots$ & .0 \\
\hline $08-25-77$ & 29 & 1200 & 1 & 2 & 5 & 40 & 660 & 10 & 50 & $\cdots$ & .0 \\
\hline
\end{tabular}

\begin{tabular}{|c|c|c|c|c|c|c|c|c|c|c|c|}
\hline $11-11-74$ & 16 & 100 & - - & $\cdots$ & . & -. & $\cdots$ & $\cdots$ & - & $\ldots$ & -. \\
\hline $12-10-74$ & 48 & 300 & 0 & 0 & 0 & 190 & 400 & 10 & - & $\ldots$ & $<.5$ \\
\hline $01-06-75$ & 16 & 140 & .. & .. & $\cdots$ & -. & .. & - & . & .. & -. \\
\hline $02-20-75$ & 20 & 130 & . & $\cdots$ & . & -. & .. & . & . & . & -. \\
\hline $03-11-75$ & 25 & 170 & 0 & 0 & 0 & 40 & 240 & 20 & $\cdots$ & .. & $<.5$ \\
\hline $04-14.75$ & 24 & 200 & -. & $\cdots$ & -. & $\cdots$ & -- & .. & $\cdots$ & .. & .. \\
\hline $05-06.75$ & 14 & 85 & .. & .. & .. & -. & - & $\cdots$ & . & . & -. \\
\hline $06.09-75$ & 24 & 190 & .. & - & $\cdots$ & $\cdots$ & $\ldots$ & - - & . & $\cdots$ & .. \\
\hline $07-0^{+}-75$ & 16 & 330 & 1 & $<10$ & 10 & 40 & 1200 & 10 & .. & .. & $c, 5$ \\
\hline $08-18 \cdot 75$ & 14 & 230 & -. & .. & -. & -. & -. & -. & $\cdots$ & . & -. \\
\hline $09-15-75$ & 16 & 270 & -. & -. & .. & -. & .. & - & . & $\cdots$ & - \\
\hline $10-15-75$ & 31 & 390 & $\cdots$ & $\cdots$ & $\cdots$ & .. & - - & .. & -. & $\ldots$ & -. \\
\hline $11-10 \cdot 75$ & 35 & 420 & -. & - & - & -- & - & $\cdots$ & - & - & .. \\
\hline $12 \cdot 15-75$ & 16 & 120 & 2 & $<10$ & 10 & 500 & 390 & 50 & - &.- & $<.5$ \\
\hline $12-22-75$ & 20 & 150 & 0 & $<10$ & 0 & 190 & 400 & 20 & 100 & .. & $<.5$ \\
\hline $01-19-76$ & 32 & 270 & -. &.- & -. & .. & -. & .. & - & .. & -. \\
\hline $02.09-76$ & 34 & 320 & -. & $\cdots$ &.- & -- & $\cdots$ & -- & . & $\cdots$ & -. \\
\hline $02-27-76$ & 21 & 160 & 1 & $<10$ & 10 & 340 & 250 & 10 & 110 & $\cdots$ & $<.5$ \\
\hline $03-03-76^{*}$ & - & 240 & . & - & $\cdots$ & 1900 & 1000 & 1200 & - & $\cdots$ & .4 \\
\hline $03-15-76$ & 30 & 260 & 0 & 0 & 0 & 40 & 970 & 10 & -. & -. & $<.5$ \\
\hline $03-16-76^{*}$ & -. & 260 & $\cdots$ & .. & - & 1200 & 1000 & 300 & .. & - & .3 \\
\hline $04-01-76^{*}$ & 25 & 180 & 0 & 0 & 70 & 4900 & 680 & 200 & $\cdots$ & 10 & .0 \\
\hline $04-06-76$ & 22 & 170 & 0 & 0 & 10 & 190 & 590 & 0 & 90 & . & $<.5$ \\
\hline $04-12-76$ & 32 & 230 & -. & .. & . & -. & -- & .. & .. & . & .. \\
\hline $04-20-76 *$ & 45 & 240 & 2 & 0 & 50 & 1800 & 1050 & 200 & $\cdots$ & $\cdots$ & .1 \\
\hline $05.05-76^{\circ}$ & -. & 260 & - & .. & .. & 700 & 550 & 0 & - & $\ldots$ & .0 \\
\hline $05-17-76$ & 36 & 290 & 0 & $<10$ & 0 & 30 & 1800 & 10 & .. & -. & $<, 5$ \\
\hline $05-19-76 *$ & - - & 300 & - & - - & - & 1600 & 1800 & 100 & . &.- & .0 \\
\hline $06-02.70 *$ & . & 300 & $\cdots$ & -. & - & 8300 & 700 & 100 & .. & - & .0 \\
\hline $06.07-70$ & 19 & 150 & -. & -. & -- & -. & - & - & . & - & .. \\
\hline $06-15-76 *$ & -. & 350 & $\ldots$ & .. & - & 1700 & $\ldots$ & $\cdots$ & .. & -. & -. \\
\hline $07-02-76 n$ & 25 & 175 & 0 & 0 & 50 & 3500 & 850 & 100 & -. & 0 & .0 \\
\hline $07-12.70$ & 17 & 190 & -. & . & -- & -. & .. & $\cdots$ & -. & -. & -. \\
\hline $07-15.76^{\mathrm{m}}$ & 20 & 175 & 1 & 20 & 20 & 3500 & 700 & 300 & $\cdots$ & 10 & .1 \\
\hline $08-09-76$ & 21 & 230 & 1 & $<10$ & 10 & 30 & 1400 & 20 & -. & .. & c. 5 \\
\hline
\end{tabular}


Table 5.--Chemical analyses of samples from Saline and Big Muddy River basins--Continued

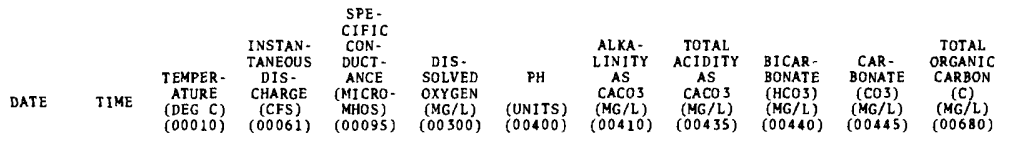

(21) *05599500 - BIG MUDDY RIVER AT MURPHYSBORO, IL (LAT 3744 SS LONG 08920 45) - Continued

\begin{tabular}{|c|c|c|c|c|c|c|c|c|c|c|c|}
\hline $08-30-76$ & 1330 & 26.0 & 57 & 1020 & 8.5 & 7.5 & 135 & 0 & 264 & 0 & $\cdots$ \\
\hline $09-13-76$ & 1530 & 20.5 & 365 & 620 & 4.1 & 7.3 & 67 & .. & 82 & 0 & .. \\
\hline $10-18-76$ & 1540 & 13.0 & 63 & 1100 & I 2.2 & 8.2 & 130 & $\ldots$ & 160 & 0 & .. \\
\hline $11-15-76$ & 1300 & 5.0 & 65 & 940 & 9.0 & 7.3 & 120 & $\cdots$ & 140 & 0 & 11 \\
\hline $12-13-76$ & 1530 & 1.0 & 126 & 1250 & 15.2 & 7.5 & $\cdots$ & - & -. & $\cdots$ & - \\
\hline $01-27-77$ & 1100 & .0 & 75 & 1280 & 6.0 & 7.2 & 160 & $\ldots$ & 290 & 0 & .. \\
\hline $02-08-77$ & 1130 & .0 & 100 & 1320 & 6.5 & 7.5 & 170 & $\cdots$ & 210 & 0 & 8.7 \\
\hline $03-22-77$ & 1400 & 11.5 & 1420 & 560 & 8.4 & 7.2 & 58 & -. & 71 & 0 & . \\
\hline $04-01-77$ & 1400 & 15.2 & 17500 & 185 & 5.1 & 7.2 & 30 & .. & 36 & 0 & 7.6 \\
\hline $05-02-77$ & 1400 & 18.0 & 808 & 600 & 8.0 & 8.0 & 66 & -. & 80 & 0 & 8.9 \\
\hline $06-01-77$ & 1000 & 25.8 & 285 & 625 & 6.3 & 7.6 & 80 & $\cdots$ & 97 & 0 & .. \\
\hline $07-11-77$ & 1545 & 28.5 & 413 & 645 & 6.3 & 7.5 & 210 & -. & 250 & 0 & 5.3 \\
\hline $08-16-77$ & 1400 & 26.0 & 937 & 395 & 4.4 & 7.1 & 36 & .. & 44 & 0 & 8.5 \\
\hline $08-29-77$ & 1200 & 24.0 & 977 & 280 & 7.5 & 7.3 & 41 & $\cdots$ & 50 & 0 & 11 \\
\hline $09-22-77$ & 1200 & 23.0 & 190 & 850 & 8.0 & 7.5 & 72 & $\cdots$ & 86 & 0 & -. \\
\hline
\end{tabular}

*. Discharge values for this station are mean daily discharges. 
Table ;..-Chemical analyses of samples from Saline and Bag Muddy Raver basins-.Continued

76

$08-30-76$

$09-13 \cdot 76$

$10-18-70$

$11-15 \cdot 76$

$12-13-76$

$01-17.77$

$02-08-77$

$03-22-77$

$04-01-77$

$05 \cdot 02 \cdot 77$

$06-01-77$

07-11-77

$08-16-77$

$08-29-77$

$09-12-77$

$\begin{array}{cccc}\text { DIS- } & & & \\ \text { SOLVED } & \text { DIS- } & \text { DIS- } & \text { SOL } \\ \text { CHLO- } & \text { SOLVED } & \text { SOLVED } & \text { C } \\ \text { RIDE } & \text { SULFATE } & \text { ARSENIC } & \text { MI } \\ \text { (CL) } & \text { (SO4) } & \text { (AS) } & \\ \text { (MG/L) } & (\text { MG } / L) & \text { (UG/L) } & (\text { UR } \\ (00940) & (00945) & (01000) & (0\end{array}$
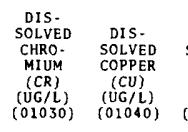

DIS- DIS.

DIS-
SOLVED DIS- SIS-
MANESELVD

MAN- SOLYED SOLVED DIS-

GANESE ZINC INUM PHENOLS MERCURY

(MN) (2N) (AL)

$\begin{array}{cccccc}(\mathrm{FE}) & (\mathrm{MN}) & (2 N) & (\mathrm{AL}) & & (\mathrm{HG}) \\ (\mathrm{UG} / \mathrm{L}) & (\mathrm{UG} / \mathrm{L}) & (\mathrm{UG} / \mathrm{L}) & (\mathrm{UG} / \mathrm{L}) & (\mathrm{UG} / \mathrm{L}) & (\mathrm{UG} / \mathrm{L}) \\ (01046) & (01056) & (01090) & (01106) & (32730) & (71890)\end{array}$

(21) 05599500 - BIG MUDDY RIVER AT MURPHYSBORO, IL (LAT 374455 LONG 08920 45)--Continued

\begin{tabular}{|c|c|c|c|c|c|c|c|c|c|c|}
\hline 35 & 420 & 1 & $<10$ & 0 & 10 & 1600 & 10 & 110 & .. & $<.5$ \\
\hline 26 & 200 & - & . & .. & $\ldots$ & . & $\ldots$ & $\ldots$ & . & . \\
\hline 43 & 420 & .. & .. & .. & .. & .. & - & $\ldots$ & .. & .. \\
\hline 48 & 310 & 1 & $<10$ & 10 & 70 & 690 & 20 & $\cdots$ & -. & $<.5$ \\
\hline .. & . & $\ldots$ & . & $\therefore$ & .. & .. & $\ldots$ & .. & - & - \\
\hline 57 & 480 & .. & $\ldots$ & $\ldots$ & $\ldots$ & .. & . & - & .. & .. \\
\hline 59 & 480 & 1 & $<10$ & 10 & 120 & 1100 & 20 & $\therefore$ & . & $<, 5$ \\
\hline 27 & 170 & .. & .. & .. & . & .. & $\cdots$ & - & $\cdots$ & .. \\
\hline 8.8 & 53 & 1 & $<10$ & 10 & 290 & 80 & 10 & 360 & .. & $<.5$ \\
\hline 33 & 180 & 1 & 7 & 2 & 10 & 20 & 0 & .. & .. & .0 \\
\hline 35 & 160 & .. & .. & .. & .. & .. & $\ldots$ & -. & $\cdots$ & .. \\
\hline 32 & 160 & 0 & 1 & 4 & so & 390 & 0 & 180 & . & .0 \\
\hline 14 & 130 & 0 & 0 & 0 & 100 & 490 & 20 & $\ldots$ & .. & .0 \\
\hline 11 & 69 & 2 & 3 & 11 & 520 & 320 & 10 & 360 & .. & .0 \\
\hline 27 & 320 & .. & .. & .. & .. & . & $\ldots$ & - & . & .. \\
\hline
\end{tabular}

A U.S. GOVERNMENT PRINTING OFFICE: $1982-361-614 / 118$ 